UC-NRLF

B 3 101 16? 


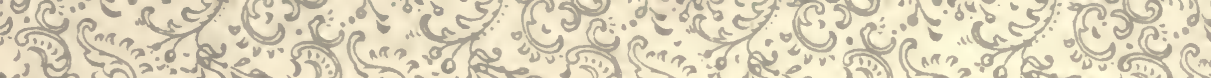

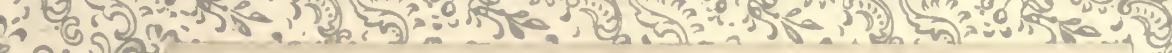

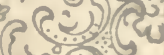

\section{UNIVERSITY OF CALIFORNIA.}

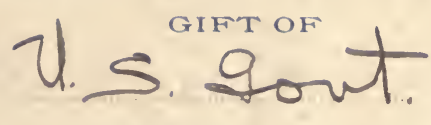

\section{Accession $\quad 99097 \quad$ Class}





\section{DEPARTMENT OF AGRICULTURE,}

\section{DIVISION OF CHEMISTRY.}

\section{TH I R D REPOR'T}

$$
\text { ON THE }
$$

\section{CHEMICAL COMPOSITION aNd PHYSICAL PROPERTIES}

\section{AMERICAN CEREALS,}

\section{WHEA'T, OATS, BARLEY, AND RYE.}

WA SH I N GTON : GOVERNMENT PRINTING OFFICE. 



\section{LETTERS OF TRANSMITTAL.}

I.

United States Department of Agridulture, Division of Chemistry, Washington, D. C., April 2, 1886.

SIR : 1 have the honor to submit herewith for your approval the final results of the investigations of American cereals which have been made by this Division under the direction of Mr. Clifford Richardson.

These investigations, it is believed, are the most extensive and thorough of any similar ones heretofore carried on, and have revealed the influence of soil and climate on the composition of our grain in a manner which will prove beneficial both to our farmers and manufacturers.

Respectfully,

H. W. WILEY,

Chemist.

Hon. N. J. Colman,

Commissioner.

II.

March $31,1886$.

SiR: I have the honor to hand you for transmission to the Commissioner of Agriculture my third report upon American cereals.

Very respectfully,

Dr. H. W. WILEY,

ULIFFORD RICHARDSON, Assistant Chemist.

Chemist. 
Digitized by the Internet Archive in 2007 with funding from Microsoft Corporation 


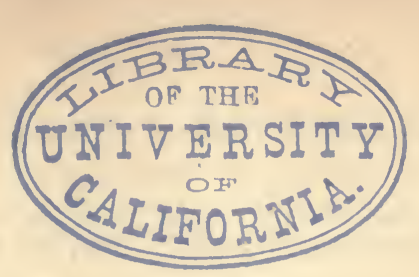

\section{COMPOSITION AND PROPERTIES OF AMERICAN CEREALS.}

\section{WHEAT.}

In previous reports, Bulletins 1 and 4 of the Chemical Division, we have discussed the changes in composition of many varieties of wheat as they have been grown in Colorado during a period of years by Prof. A. E. Blount. His attempts have been to improve by selection and crossing the character of this grain both for the farmer and miller. What success he has met with in increasing the yield and physical characteristics will be evident from the data which follow. It has been our province to study the changes in the chemical composition of the grain from year to year, showing the result of varying conditions in this direction. This has been done for four years, and before giving the results of the last year's examination a summary of those of previous years will serve to make them more intelligible.

The first specimens of wheat were handed to the Dirision by Professor Blount in 1881, and were of varieties which had been grown two and three years in Colorado. They numbered thirty-three, and had the following arerage composition:

\begin{tabular}{|c|c|}
\hline Weight of 100 grains. & $\begin{array}{c}\text { Grams } \\
4.865\end{array}$ \\
\hline 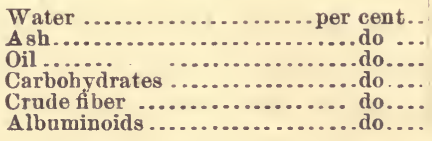 & $\begin{array}{r}9.86 \\
2.28 \\
2.41 \\
70.48 \\
1.57 \\
13.40\end{array}$ \\
\hline Total......... & 100.00 \\
\hline Nitrogen.... & 2.14 \\
\hline
\end{tabular}

From this arerage it was learued that in that year Colorado produced a grain very rich in albuminoids, large in size, dry, and with little fiber or hull.

Among the specimens it was found that some were from domestic and others from foreign seed, part being Russian. Knowing that Russian wheat is the richest in albuminoids of any in the world, and that continental varieties as a rule contain more than our own, it wa: of interest to 
observe that the crops grown in Colorado were still characterized by the source of the seed.

Average composition of crops from seed from various sourcts.

\begin{tabular}{|c|c|c|c|}
\hline & $\begin{array}{l}\text { Domestic } \\
\text { seed. }\end{array}$ & $\begin{array}{l}\text { Foreign } \\
\text { seedl. }\end{array}$ & $\begin{array}{l}\text { Russian } \\
\text { seed. }\end{array}$ \\
\hline 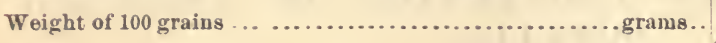 & 4. 714 & 5. 187 & 5. 075 \\
\hline 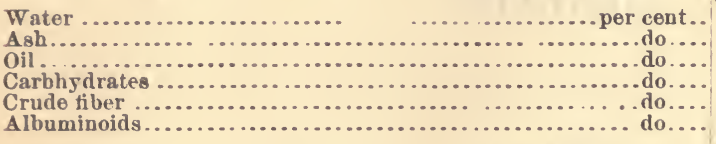 & $\begin{array}{r}9.85 \\
2.27 \\
2.38 \\
70.87 \\
1.58 \\
13.05\end{array}$ & $\begin{array}{r}9.86 \\
2.32 \\
2.45 \\
69.46 \\
\text { i. } 57 \\
14.34\end{array}$ & $\begin{array}{r}9.69 \\
2.41 \\
2.41 \\
69.33 \\
1.59 \\
14.54\end{array}$ \\
\hline 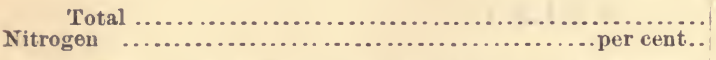 & $\begin{array}{r}100.00 \\
2.09\end{array}$ & $\begin{array}{r}100.00 \\
2.29\end{array}$ & $\begin{array}{r}100.00 \\
2.32\end{array}$ \\
\hline
\end{tabular}

These wheats were the richest in albuminoids of any that had been examined up to that time in this country. Since then the bard spring wheats of the North west have been found to be more nitrogenous, but not comparable in their size or yield.

The second year, twelve varieties, grown from seed supplied by the Department of Agriculture, were analyzed. $\Lambda$ fter one year, that is to say, the first year's growth in Colorado, they were found to have all increased in size, and instead of an average weight of 3.402 grams per hundred grains, they weighed 4.299 grams. As regards the percentage of albuminoids, where the seed wheat was low in nitrogen there was a gain, but as half of the varieties contained originally more albuminoids than the average Colorado grain, there was a drop in six of the twelve specimens toward the average. For example, a seed having 16.11 per cent. of albuminoids, the crop fell to 1491 per cent., while one having only 9.65 rose to 12.15 per cent. From these facts, aud the analyses of the previous year, the conclusion was drawn that the conditions in Colorado were suited to the production of a grain containing about 13 per cent. of albuminoids.

This was undoubtedly the case at the time. In the two following years, however, these conditions have been somewhat modified.

In the third year, 57 varieties were examined, 28 of which had been analyzed before in 1881. A loss of albuminoids occurred in all but 4 cases, and a loss of weight in all. The average for the year was-

\begin{tabular}{|c|c|}
\hline Weight of 100 grains. & $\begin{array}{c}\text { Grams } \\
3.941 \text {. }\end{array}$ \\
\hline 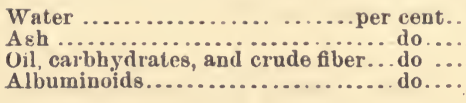 & $\begin{array}{r}9.38 \\
2.09 \\
\text { 76. } 79 \\
11.74\end{array}$ \\
\hline 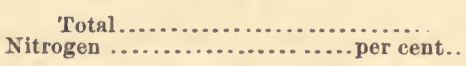 & $\begin{array}{r}100.00 \\
1.88\end{array}$ \\
\hline
\end{tabular}


This sudden change and drop in size and percentage of albuminoids were attributed by Professor Blount to a heavy hail-storm, which prostrated the crop in its formation stage and influenced all its characteristics. This was probably the case, and it became of great interest to study the crop of 1884, to learn what the recovery might be, and what, after from one to six years' grow th in Colorado, the changes in average composition might amount to.

To this end 77 varieties, selected from a lot of 200 grown by Professor Blount, have been examined chemically and physically, and the results are presented in the following tables, together with certain data in regard to yield per acre, as well as characteristics of the seasons and other conditions, taken from Professor Blount's report and personal letters. 


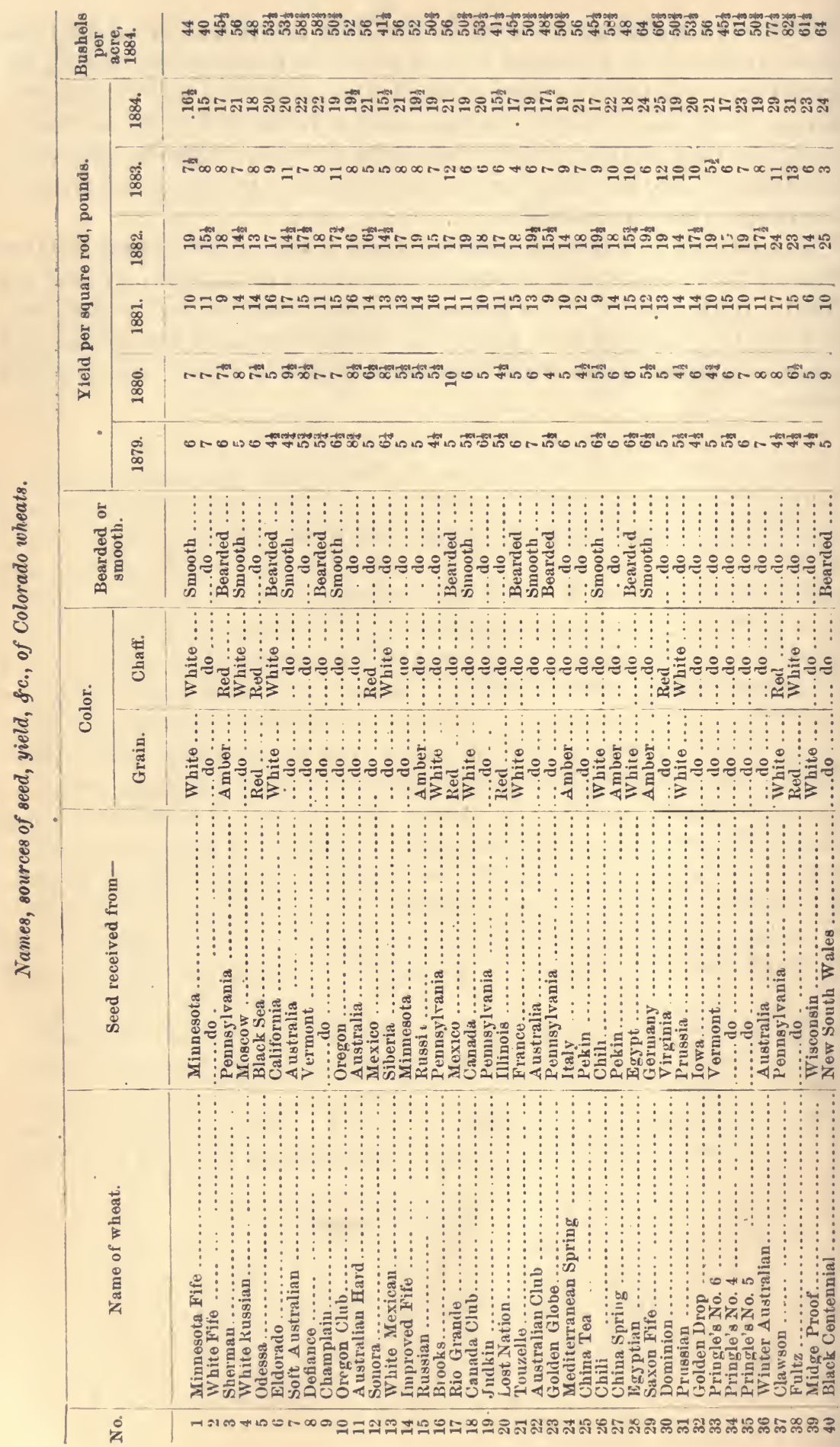




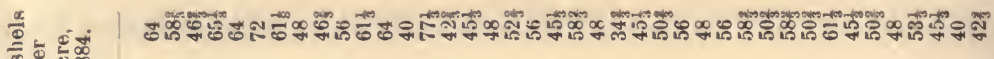
$\approx-\pi$

வ

\begin{tabular}{|c|c|}
\hline$\stackrel{\leftrightarrow}{\mathscr{\infty}}$ & ปลิ \\
\hline 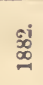 & 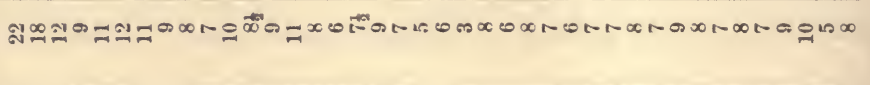 \\
\hline$\dot{\infty}_{\infty}^{\infty}$ & ম̋ \\
\hline$\underset{\substack{\infty \\
: 0}}{ }$ & \\
\hline & : \\
\hline
\end{tabular}

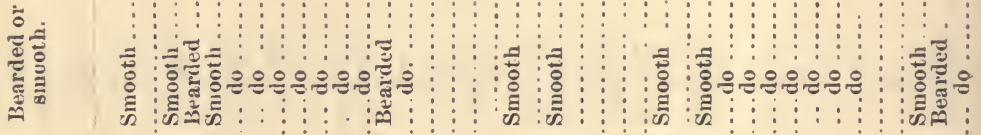

ปัँ

$\dot{\overline{0}}$

苞

ชั.

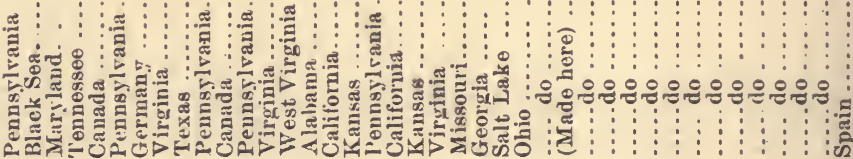

$\frac{\dot{m}}{\ddot{m}}$

5

莺

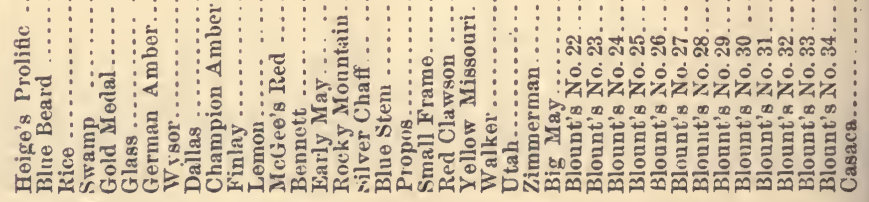

そ่ | 


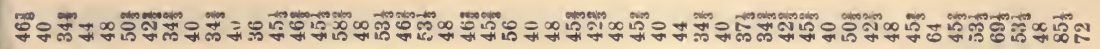

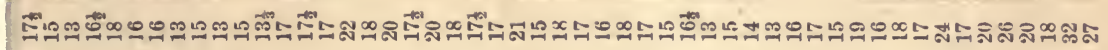

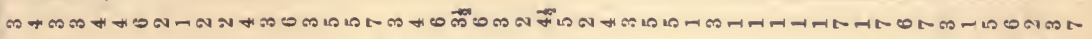

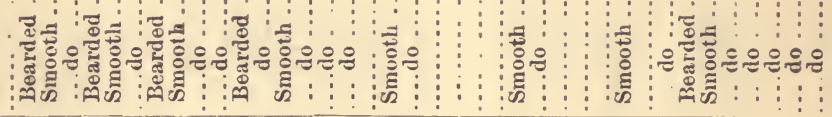

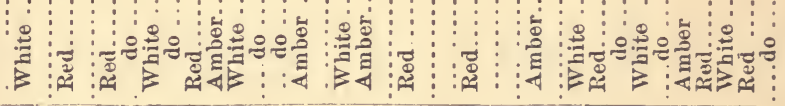

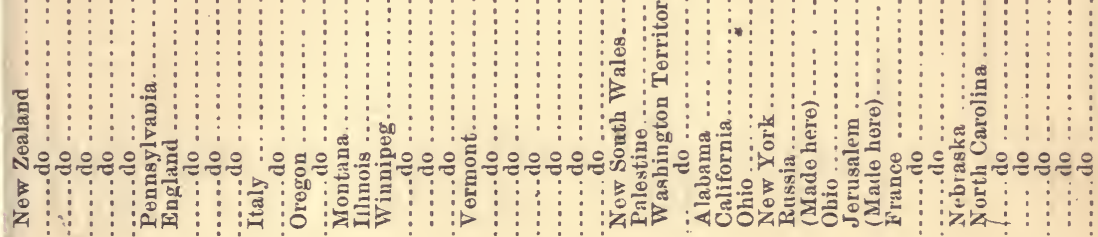

0
4
4

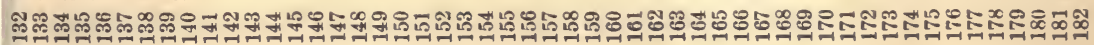




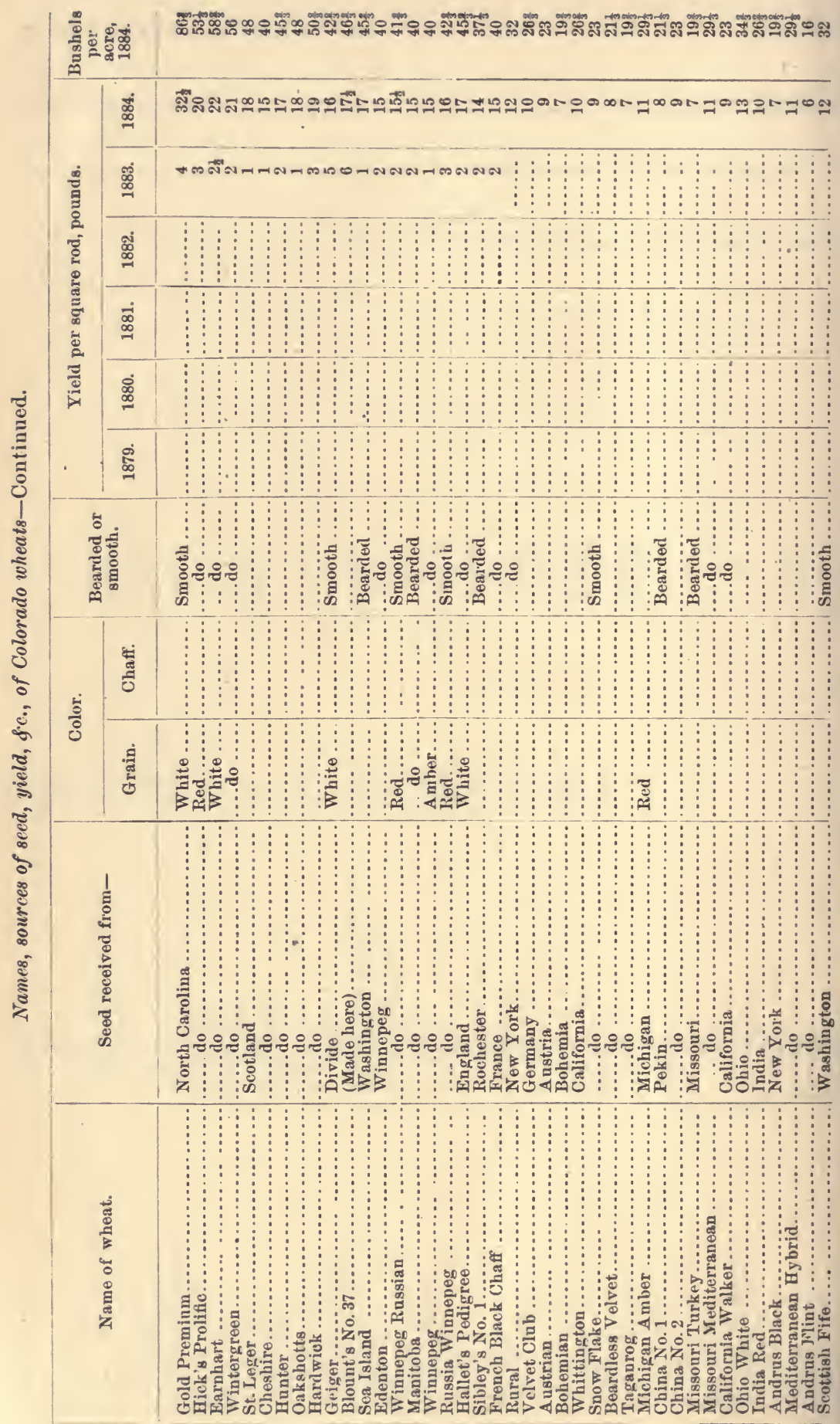

| 


\section{Of his method of cultivation Professor Blount says:}

It is quite simple. I sow only one onnce of picked seed per square rod in eight rows, or in field culture only thirty pounds per acre. On each square rod I put homemade fertilizers, horse, cow, hog, and sheep manure, the latter being by far the best in this climate. In the case of cow and hog manures, one cubic foot is nsed, but two cubic feet of horse manure. There are also plots with no nianures. My crops are hoed twice and irrigated twice.

\section{Of the seasons he says :}

1879 was a fair wheat year, no rain from April to harvest; 1880 two showers, doing more damage to wheat than none; $18 \varepsilon 1$ and 1882 fair wheat seasons; 1833 hail killed everything, and 1884 a fine season with some rain.

A description of his methods of crossing and selection will be found in the Anuual Report of this Department for 1881-'82, and in the report of the agricultural department of the Colorado State College for 1884.

In addition it is necessary to say that it must be borne in mind that these wheats have been grown upon an experimental scale and with greater care and regularity of condition than conld be often found in field culture in this country. While they do not represent, therefore, the ordinary product of the State from which they come, they are more valuable for purposes of scientific comparison and as a guide to what may be done by the farmer in the improvement of his seerl aud crops.

Physical properties of Colorado wheat, 1884.

\begin{tabular}{|c|c|c|c|c|c|c|c|}
\hline Name. & 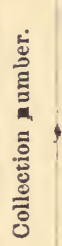 & 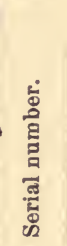 & Color. & 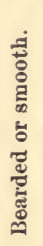 & 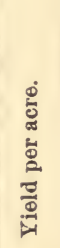 & 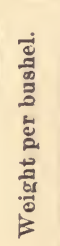 & 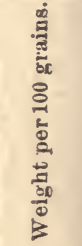 \\
\hline Oregon Club. & & & & & Bus. & Lbs. & Grains. \\
\hline Aregon Club Ha... & 11 & $\begin{array}{l}3500 \\
3.001\end{array}$ & $\begin{array}{l}\text { Light amber. } \\
\text {....do ......... }\end{array}$ & S. & $\begin{array}{l}50 \frac{9}{8} \\
52\end{array}$ & $\begin{array}{c}63.7 \\
\ldots . . .\end{array}$ & $\begin{array}{l}3.647 \\
4.041\end{array}$ \\
\hline Sonora.............. & 12 & 3502 & Yellow ......... & S. & 56 & 67.3 & 3. 830 \\
\hline White Mexican ................ & 13 & 3503 & ....do .... & s. & $41 \frac{1}{3}$ & 63.5 & 4.890 \\
\hline Improved Fife................... & 14 & 3504 & Light amber ............ & S. & $56^{3}$ & 65. 8 & 3. 672 \\
\hline Brooks $\ldots \ldots \ldots \ldots$ & 16 & 3505 & A mber..................... & S. & $50 \frac{9}{3}$ & 65.0 & 3. 841 \\
\hline Rio Grande.................... & 17 & 3506 & $\ldots$ do ..... & B. & 56 & 67.1 & 4. 743 \\
\hline Canada Club.................... & 18 & 3507 & ... do ................... & S. & $50 \frac{2}{3}$ & 66.1 & 3. 764 \\
\hline ............... & 19 & 3508 & Dark amber & S. & $53 \frac{1}{3}$ & 64.1 & 3. 920 \\
\hline Lost Nation. . & 20 & 3509 & A mber........... & S. & $41 \frac{1}{3}$ & 64.5 & 4. 147 \\
\hline Touzelle ....... & 21 & 3510 & Lighter ainber ......... & B. & $45 \frac{1}{3}$ & 65.7 & 4. 300 \\
\hline Australian Club................. & 22 & 3511 & Amber white ........... & S. & $50 \frac{2}{3}$ & 64.8 & 4. 536 \\
\hline Golden Globe....... & 23 & 3512 & A mber........ & B. & $48 \frac{2}{3}$ & 66.5 & 4670 \\
\hline Mediterranean Spring......... & 24 & 3513 & .... do ...... & B. & $50 \frac{3}{3}$ & 66.2 & 4. 640 \\
\hline China Tea ........................ & 25 & 3514 & do .... & B. & $56^{\circ}$ & 67.0 & 5.000 \\
\hline ........ & 26 & 3515 & Yellow & S. & $45 \frac{3}{3}$ & 64.9 & 4. 440 \\
\hline China Spring ................ & 27 & 3516 & Dark anuber... & S. & $58 \frac{8}{3}$ & 65.4 & 3.990 \\
\hline Egyptian Fife $\ldots \ldots \ldots \ldots \ldots$ & 28 & 3517 & Yellow amber ......... & B. & 48 & 63. 8 & 4. 840 \\
\hline Saxon Fife.................... & 29 & 3518 & Red & S. & 64 & 65.3 & 3. 690 \\
\hline 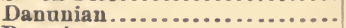 & 30 & 3519 & Red and yellow. & S. & 66 ? & 62.5 & 4. 110 \\
\hline Prussian................... & 31 & 3520 & Dark amber .... & S. & $50 \frac{2}{3}$ & 64.0 & 3. 610 \\
\hline ............ & 34 & 3521 & Light amber........... & S. & $45 \frac{3}{8}$ & & 4. 300 \\
\hline Hedge Row, White Chaff...... & 41 & 3522 & Yellow amber & S. & 48 & 65.9 & 3.170 \\
\hline Italian $\ldots \ldots \ldots \ldots \ldots \ldots \ldots$ & 43 & 3523 & Red and yellow...... & B. & $45 \frac{1}{3}$ & 64.1 & 5.620 \\
\hline Hybrid No. $10 \ldots . .$. & 51 & 3524 & Pale yellow... & S. & $61 \frac{3}{3}$ & 63.9 & 4. 690 \\
\hline Nox No.1.... & 54 & 3525 & Yellow and amber.... & S. & $50 \frac{2}{3}$ & 64.8 & 3. 980 \\
\hline Hybrid No. & 56 & 3526 & Ámber.................. & S. & $45 \frac{1}{3}$ & 62.7 & 3160 \\
\hline 15. & 57 & 352 & Yellow an & B. & 56 & 63.6 & 3.200 \\
\hline Hyb & 58 & 3528 & Red aud amber ....... & S. & 40 & 62.8 & 4. 110 \\
\hline Hybrid No. 17..... & 59 & 3529 & Red................. & B. & 56 & 66.8 & 4. 740 \\
\hline
\end{tabular}


Physical properties of Colorado wheat, 1884-Continued.

Andriola Amber

Big Mars

Hybrid No. 26

Hybril No. $28 \ldots \ldots \ldots \ldots \ldots \ldots$

Gybrid No. 33

Horid No. 34 -...

Monmouth.................... 148

Russiau Spring .............. 149

Vermillion .................. 150

Edeuton Fife................. 152

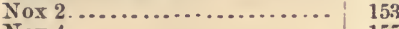

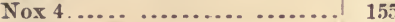

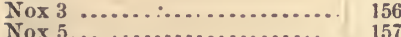

Nox $5 . \ldots \ldots \ldots \ldots \ldots . .15$

Wiles......................... 160

Northeote's Imperial....... 162

Northcote's Amber ........... 163

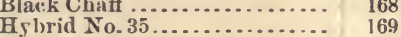

Hebron .................... 171

Mediterranean White....... 173

Frenteh Inperial....... .... 175

Nebraska ... ............. 176

Northcote's White .......... 177

Kiret $\quad \ldots \ldots \ldots \ldots \ldots \ldots \ldots$. 178

Baltimore ............... 180

Daris $\quad . . . . . . . . . . . . .181$

Gold Premium .............. 183

Hick's Prolific .............. 184

Wintergreen ............... 186

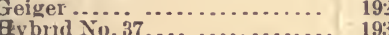

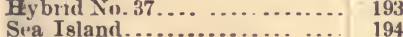

Edenton .................. 195

Winnipeg, Russian.......... 196

Manitoba.................... 197

Winnipeg ...... ......... 198

Hallıtt's Pedigree ... . ....... 200

China No. 2 ................ 213

Mo. Turkey ................. 214

Mo. Mediterranean .......... 215

Scotch Fife $\quad . . . \ldots \ldots \ldots \ldots . .229$

Rye .....................

Sandomirka ................

Hopetown .................. 235

\begin{tabular}{|c|c|c|c|c|c|c|}
\hline 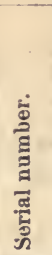 & Color. & 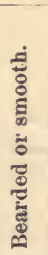 & 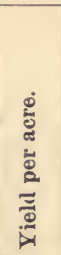 & 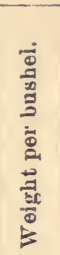 & 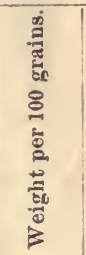 & 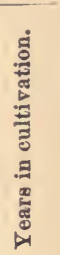 \\
\hline 530 & Red and amber... & B. & $\begin{array}{r}\text { Bus. } \\
61 \frac{1}{3}\end{array}$ & $\begin{array}{l}\text { Lbs. } \\
66.4\end{array}$ & $\begin{array}{c}\text { Grains. } \\
3.790\end{array}$ & \\
\hline 531 & Dark amber ............ & s. & $58^{\frac{3}{3}}$ & 66.0 & 3.660 & \\
\hline 3532 & Dark yellow .......... & S. & 56 & 63.5 & 4.710 & \\
\hline 3533 & Light amber....... & S. & $58 \frac{9}{3}$ & 65.9 & 5.339 & \\
\hline 3534 & Dark yellow........... & S. & $61 \frac{1}{2}$ & 62.2 & 4. 683 & \\
\hline 3535 & do & s. & $45 \frac{1}{3}$ & 62.8 & 3.586 & \\
\hline 3536 & Glassy amber ... & B. & $40^{\circ}$ & 64.4 & 6. 620 & 3 \\
\hline 3537 & Red & B. & $42 \frac{8}{3}$ & & 3.299 & \\
\hline 3538 & Light red. & B. & $48^{3}$ & 67.0 & 4.830 & \\
\hline 3539 & Red.................. & S. & $53 \frac{1}{3}$ & 64.3 & 3.880 & \\
\hline 3540 & ....do. & S. & $46 \frac{2}{3}$ & 64.9 & 3.500 & \\
\hline & .. do. & S. & 48 & 63.5 & 4.100 & \\
\hline 3542 & Yellow amber ........ & S. & $46 \frac{2}{3}$ & 65.7 & 4. 170 & \\
\hline & Light red & B. & 56 & 66. 1 & 4. 670 & \\
\hline 3544 & auber .......... & B. & 40 & 67.2 & 5. 535 & 3 \\
\hline 5 & Yellow amber......... & S. & 48 & 65.0 & 4. 239 & \\
\hline 6 & $\therefore$ do . & S. & $45 \frac{2}{3}$ & 64.2 & 4. 167 & \\
\hline 7 & Light am & s. & $48^{3}$ & 64.6 & 5. 073 & \\
\hline 8 & Yellow & s. & 40 & 63.7 & 3.576 & \\
\hline 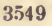 & Light red & S. & 44 & 64.1 & 4.120 & \\
\hline 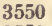 & Red & S. & $42 \frac{2}{3}$ & 66.7 & 3.420 & \\
\hline 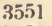 & A inber yellow ........ & S. & $45 \frac{3}{3}$ & 62.2 & 3.800 & \\
\hline 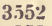 & do... & & $50 \frac{2}{3}$ & 63.9 & 3.500 & \\
\hline 3 & Deep yellow ....... & s. & $48^{3}$ & 66.9 & 3.580 & \\
\hline & Red & s. & 64 & 66. 6 & 4.880 & \\
\hline $3557>2>$ & Amber. & B. & $45 \frac{2}{3}$ & 66.4 & 4. 440 & \\
\hline & ........... & S. & $53 \frac{3}{3}$ & 64.6 & 4.400 & \\
\hline 355 & Deep yellow ... & s. & $69 \frac{1}{3}$ & 6.5. 0 & 4.220 & 2 \\
\hline & Light red. & S. & $48^{3}$ & 67.1 & 5.060 & \\
\hline & do & s. & $85 \frac{1}{3}$ & 66.1 & 4.220 & \\
\hline & do & S. & 86 & 63.7 & 4. 120 & \\
\hline & Red. & S. & $53 \frac{1}{3}$ & (66. 1 & 3.890 & \\
\hline & A inber. & S. & $56^{\circ}$ & 66.9 & 3.930 & \\
\hline & ..... do & S. & 4 & & 4. 240 & \\
\hline & Amber yellow & & $46 \frac{2}{3}$ & 64.2 & 3.850 & \\
\hline & Red & B. & $45 \frac{\mathrm{g}}{3}$ & & 3.420 & \\
\hline & $\mathrm{Am}$ & B. & $40^{3}$ & 65.6 & 5.180 & \\
\hline & Ligl & s. & $+1 \frac{2}{3}$ & 64.4 & 4.120 & \\
\hline & $\mathrm{R}+\mathrm{d}$ & B. & 40 & 66.3 & 3.580 & 2 \\
\hline & Glas: & B & 40 & 67.8 & 5. 560 & \\
\hline 3570 & A mber and yellow..... & S. & $45 \frac{2}{3}$ & 64.5 & 3.880 . & 2 \\
\hline 357 & Amber & & 24 & 67.7 & 3.180 & \\
\hline 3.7 & , & B. & $18 \frac{2}{3}$ & ...... & 4.000 & 1 \\
\hline & Light red. & B. & $29 \frac{1}{3}$ & …... & 4.480 & \\
\hline & Red & s. & $32^{\circ}$ & 64.6 & 3.440 & 1 \\
\hline & Dark and light red.... & S. & $21 \frac{1}{3}$ & 64.7 & 4. 760 & \\
\hline $357 .>2>$ & red ............ & & & 68.6 & 4.060 & \\
\hline 357 & Amber... & & & 67.0 & 4.500 & 1 \\
\hline
\end{tabular}




\begin{tabular}{|c|c|c|}
\hline \multicolumn{2}{|c|}{ 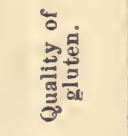 } & ¿ूँ \\
\hline \multirow{2}{*}{ 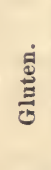 } & $\dot{\vec{A}}$ & 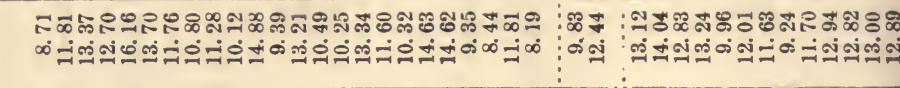 \\
\hline & $\frac{\dot{n}}{\frac{\pi}{2}}$ & 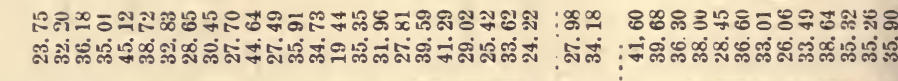 \\
\hline \multicolumn{2}{|c|}{ 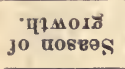 } & 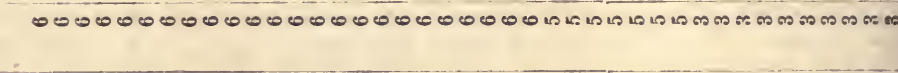 \\
\hline \multicolumn{2}{|c|}{ 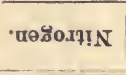 } & 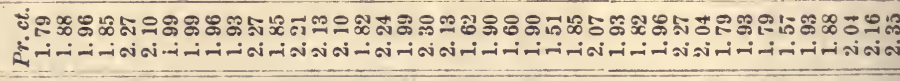 \\
\hline \multicolumn{2}{|c|}{$\begin{array}{l}\text { 'spṭo } \\
\text {-u!̣unqเ }\end{array}$} & 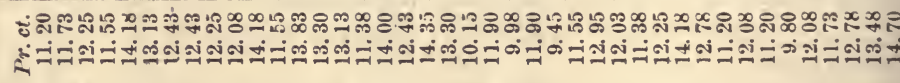 \\
\hline \multicolumn{2}{|c|}{ :เәфฺ!. } & 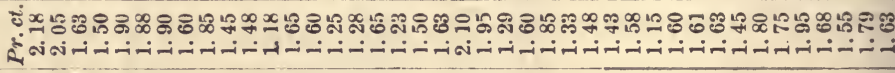 \\
\hline \multicolumn{2}{|c|}{ 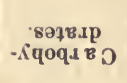 } & 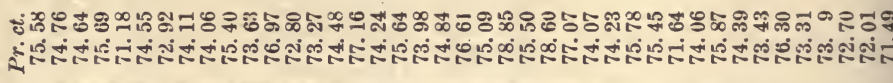 \\
\hline & ‘เด & 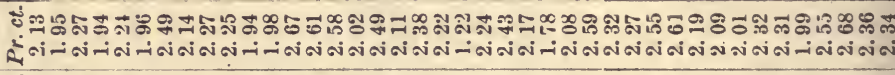 \\
\hline & "ys & 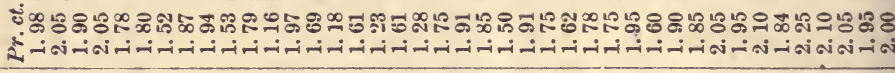 \\
\hline \multicolumn{2}{|c|}{ 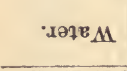 } & 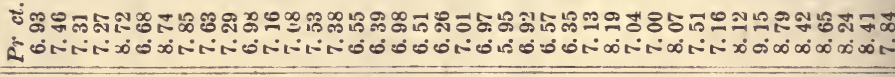 \\
\hline \multicolumn{2}{|c|}{ 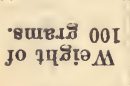 } & 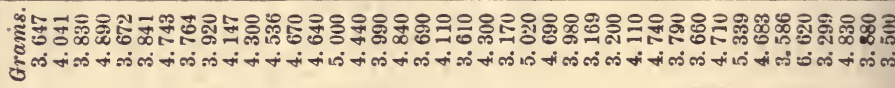 \\
\hline . & & $\begin{array}{c}\vdots \\
\vdots \\
\vdots\end{array}$ \\
\hline
\end{tabular}

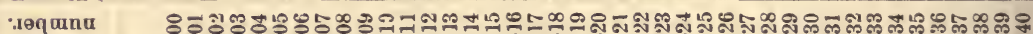
[EIIAS

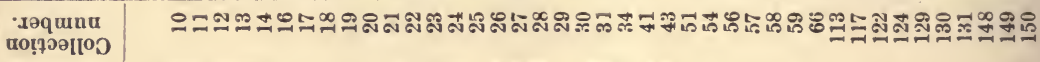




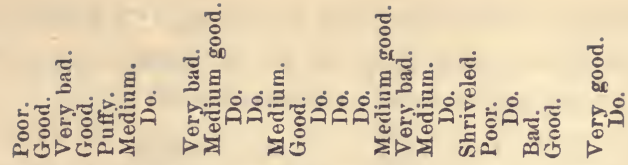

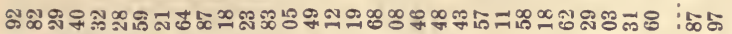

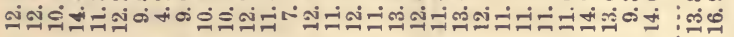

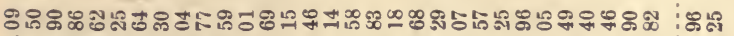

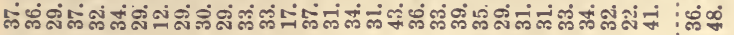

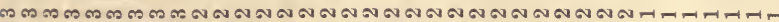

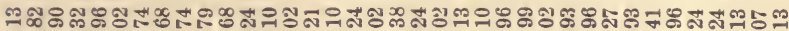

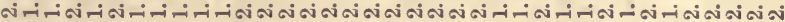

లో

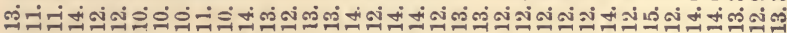

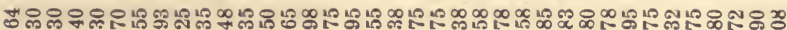

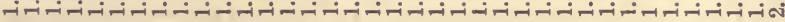

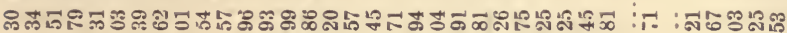

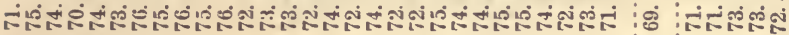

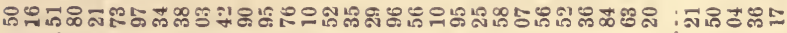

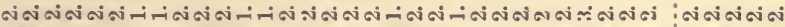

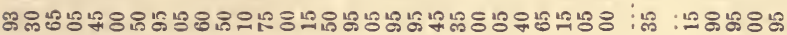

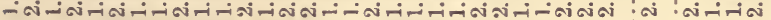

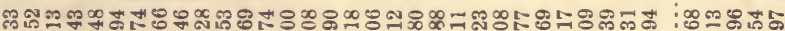

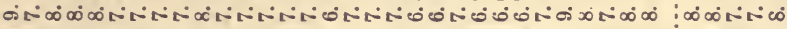

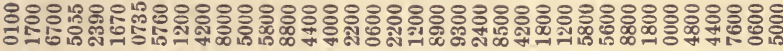

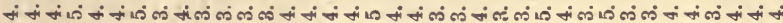

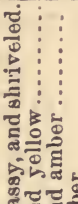

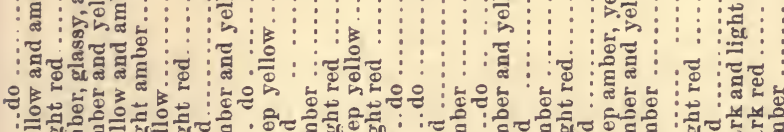

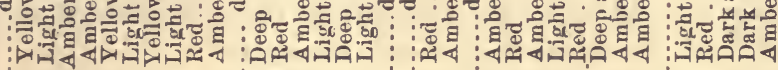

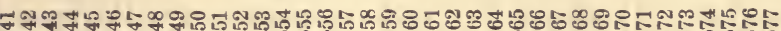

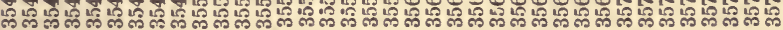
ำ

क

:

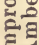

$\sum_{\mathscr{L}}^{\infty}$

:

$\vdots \vdots:=$

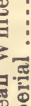

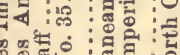

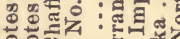

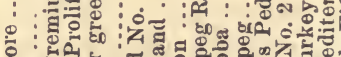


The data in the precelling tables have been areraged for comparison with the arerages of previous years as well as of those wheats which had been grown different lengths of time in Colorado.

deerage composition of Colorado wheat grown in 1884.

All varieties...

$$
\text { हूँ }
$$

Sixth season....

Fifth season....

Third season ...

Second season...

|

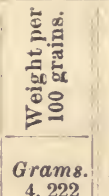

77
24
7
19
21
7

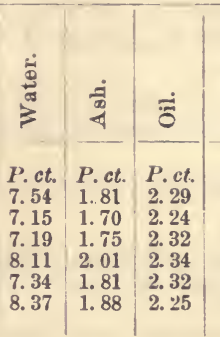

\begin{tabular}{|c|c|}
\hline 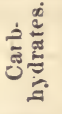 & $\frac{\dot{a}}{\dot{s}}$ \\
\hline P.ct. & P.ct. \\
\hline 74. 19 & 1.64 \\
\hline 74.83 & 1. 64 \\
\hline 75. 27 & 1. 49 \\
\hline 73. 90 & 1. 59 \\
\hline 73. 96 & 1. 67 \\
\hline 71.90 & 1.83 \\
\hline
\end{tabular}

P.et.
12.53
12.44
11.98
12.05
12.90
13.77

\begin{tabular}{|c|c|c|}
\hline \multirow{2}{*}{ 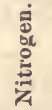 } & \multicolumn{2}{|c|}{ Gluten. } \\
\hline & Muist. & Dry. \\
\hline P.ct. & $P . c t$. & $P$. ct. \\
\hline 2.00 & 33. 31 & 10.42 \\
\hline 1. 99 & 32. 96 & 10. 31 \\
\hline 1. 92 & 35.75 & 12.45 \\
\hline 1. 93 & 34. 33 & 12. 05 \\
\hline 2.06 & 32.50 & 11.65 \\
\hline 2. 20 & 42. 68 & 15. 15 \\
\hline
\end{tabular}

The average for the seventy-seven varieties grown in 1884 when compared with that of other years shows that in size and percentage of albuminoids, although there has been an adrance over 1883 , the wheats of that year are not equal to those of 1881 and 1882.

Average composition of Colorado wheats.

\begin{tabular}{|c|c|c|c|c|}
\hline - & 1881. & 188. & 1883. & 1884. \\
\hline Weight of 100 grains......... & 4.865 & 4. 283 & 3. 941 & 4.222 \\
\hline $\begin{array}{l}\text { Water } \\
\text { Ash } \\
\text { Oil } \\
\text { Carbhydrates } \\
\text { Crude fiber } \\
\text { Albuminoids }\end{array}$ & $\begin{array}{r}9.86 \\
2.28 \\
2.41 \\
70.48 \\
1.57 \\
13.40\end{array}$ & $\begin{array}{r}8.80 \\
1.99 \\
2.38 \\
72.03 \\
1.76 \\
13.04\end{array}$ & $\begin{array}{r}9.38 \\
2.09 \\
76,79 \\
11.74 \\
\end{array}$ & $\begin{array}{r}7.54 \\
1.81 \\
2.29 \\
74.19 \\
1.64 \\
12.53\end{array}$ \\
\hline Total..................... & 100.00 & 100.00 & 100.00 & 100.00 \\
\hline $\begin{array}{l}\text { Nitrogen } \\
\text { Moist gluten } \\
\text { Dry gluten } \\
n_{n}\end{array}$ & $\begin{array}{r}2.14 \\
33.12 \\
11.74\end{array}$ & $\begin{array}{r}2.09 \\
34.69 \\
12.89\end{array}$ & 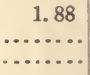 & $\begin{array}{r}2.00 \\
33.31 \\
10.42\end{array}$ \\
\hline
\end{tabular}

It would perhaps be unsafe to draw any definite conclusions from these arerages, but they would seem to show that peculiarities of sea. son are most influential on the composition of the grain.

Examined in connection with the individual analyses and with regard to the characteristics of the sereral seasons of growth it would appear that the low percentage of albuminoids in 1884 may be due to the fact that having fallen to 11.74 in 1883 , the wheats were unable to recorer more than to 12.53 in 1884 . If this is the case the effects of this bad season and set back in 1883 may be overcome in 1885. Analysis of that year's crop will decide this.

Then, the inquiry may be made as to what influence on this average is due to the continued growth of the grain on one soil for a number of years. To examine this question the averages by seasons of growth were calculated. From these one learns that the wheats of the first two seasons growth are richer in albuminoids than those which have been raised a longer time in Colorado. In the case of the wheats grown for 
the first time in the State the higher figures are probably dne to the fact that the seed had not been injured by the hailstorm which hal affected the others, and they therefore produced a grain as rich as that from seed introduced in 1881 and 1853. Why, however, there should be a decrease from the second to the fifth season and increase in the sixth is not so easy to say. The difference is small and may be due to peculiarities in the rarieties rather than other conditions.

To discover what the deterioration may have been for particular varieties the analyses of all wheats which have been made more than once are tabulated together on the following page:

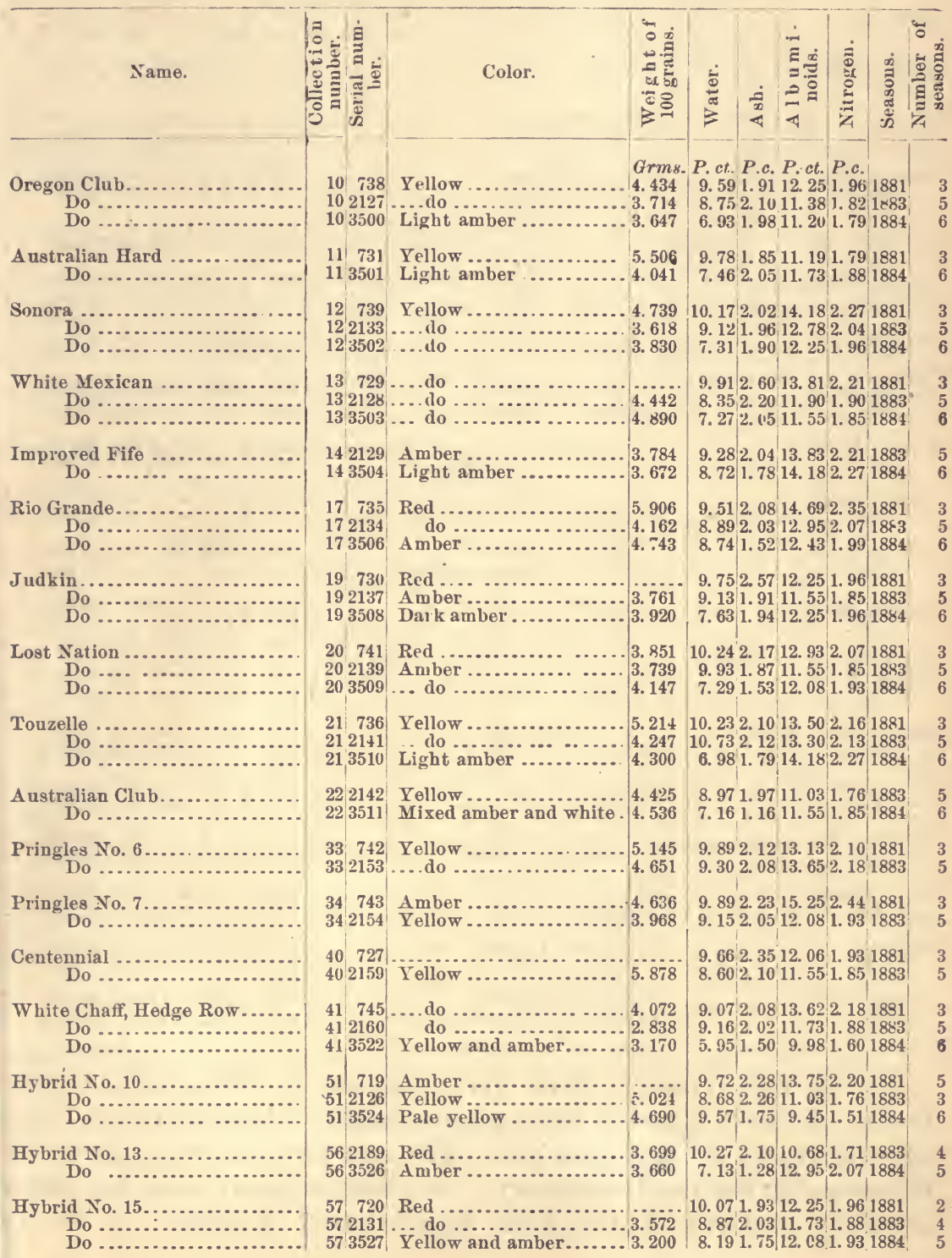




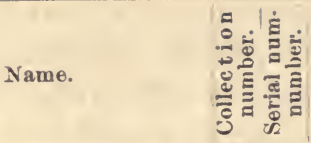

Hybrid No. 16.

Do

Do $\ldots . . . \ldots \ldots \ldots \ldots . . . . .$.

Bybrid No. 17 .

Do

Hedge Row, Red Chaff

Do

Fountain

Do .

White Chaff Do

Perfection

$$
\text { Do. }
$$

German Fife.$$
\text { Do }
$$

Triticum

$$
\text { Do ................ }
$$

Russian Durum Do

Meekin's Do

Hybrid No. 26 Do

Hybrid No. 28. Do

Hy brid No. 33 Do

Uy brid No. 34 Do

Rnssian, Spring .

$$
\text { Do }
$$

Hybrid No. 35 Do

Mediterranean White Do

French Imperial Do

Gold Premium Do

Hick's Prolific Do

Geiger

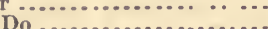

Hybrid No. 37 Do
$58 \quad 7: 1$ Red

583528 Red and amber......... 4.110

59722 Amber

592135 Red.

593529

5.137

69746 Amber

692161 Yellow

$\begin{array}{lll}71 & 732 & \ldots . .\end{array}$

$712162 \ldots . . .10$

\begin{tabular}{ll|l}
74 & 717 & Red
\end{tabular}

4. 818

4. 740

742163 . do

4. 499 4. 499
4. 008

5. 100 4. 191

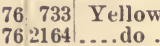

4. 214

\begin{tabular}{l|l|l|}
77 & 737 & Red
\end{tabular}

77.2168 Ambe

79 748 Yellow

792165 . . . do

81 749 Anber

812166 ' Yllow

88 751 Red

882167 .....do

1222146 Yellow

1223533 Light amber

1242148 Yellow

1243534 Dark yellow

$1 \div 92152$ Yellow 1293535 Dark yellow

5.536

1302155 Amber ................ 5. 179

130 3536 Amber and glassy.......6.620

1492171 Amber ................ 3. 438

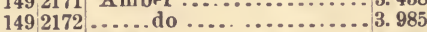

1493539 Red ................. 3.880

1692156 Yellow ................ 055 1693551 Amber and yellow ..... 3.800

1732174 Yellow ................4.182

1733553 White $. . . \ldots \ldots \ldots \ldots . . .5 .580$

1752178 Amber ................. 4. 594

1753554 Red .................. 4.880

1832184 Yellow ....................... 818

1833560 Light red ................ 4. 120

1842186 Amber .............. 2.879

1843561 Red ................... 3. 890

1922188 Yellow ............... 4. 064 1923563 Amber ................. 4.240

1932158 Yellow.

1933564 A mber and jellow \begin{tabular}{r|r|r|r|r|r|}
\hline 3.559 & 10.72 & 2.44 & 11.90 & 1.90 & 1883 \\
3.850 & 6.08 & 2.05 & 12.20 & 1.96 & 1884
\end{tabular}

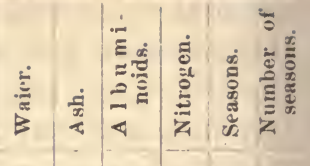

P.ct. P.c. P.ct. P.c.

9.532.04 11.75.1.88.1881 2

702. 1311.031 .761883

7. 44 I. 95 11. 38 1. 821884

$9.932 .0713 .62 \cdot 2.181881$

8. 90 ㄴ. $23 \quad 14.352 .301883$

7. 00 i. $6012.25 \quad 1.961884$

$9.17 \cdot 5912.942 .0711881$

9. 1$\} \quad 2.19$ 12.95:2.07 1883

10. 5 \& 2.7013 .622 .181881 $8.2 \%$ ?. 1411.90 1.90,1883

$9.57 \div 2.0314 .042 .251881$ 7. 95 2. 0512.081 .931883

9. 931.9914 .182 .271881 10.292.08 12.95 2.07 1883

\begin{tabular}{ll|l|l|l|l|l|l|}
0.42 & 2.31 & 15.062 .41 & 1881
\end{tabular} 10.052 .2812 .602 .021883

\begin{tabular}{ll|l|l|l|l|}
0.02 & 2.67 & 13.62 & 2.18 & 1881
\end{tabular} 8. $982.0 \geq 14.00: 241883$

9. 91 2. 32 15. 25 2. $44 \quad 1881$ $8.70 \cdot 2.1014 .352 .301883$

9. 38.2 .5315 .152 .431881 10. $152.0513 .482 .16 \quad 1883$

\begin{tabular}{ll|l|l|l|l|l|}
9.40 & 2.20 & 14.38 & 2.38 & 1883
\end{tabular} 8. 121.9512 .081 .931884

9. 32 2. 28 9.98 1.601883 9. 15 2. 1011.20 1. 791884

\begin{tabular}{ll|l|l|l|l|l|l|l|l|}
10.15 & 1.87 & 8.93 & 1.43 & 1883
\end{tabular}

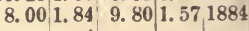

8. 822.4312 .602 .021883 8. 422.2512 .081 .931884

8. 922.3112 .782 .041882 9.682 .1412 .251 .961883 8. 411.9513 .482 .161884

9.372 .2710 .501 .681883 7. 531.5010 .501 .681884

9. 692.1711 .201 .791883 7. 74 1. 7513.132 .101884

9. $551.9512 .952 .0718 \varepsilon 3$ 7. 002.0012 .602 .021884

9.442 .1711 .381 .821883 6. 801.9514 .002 .24 .1884

9. 212.0410 .331 .651883 6. 88 1. 4512.78 2. 021884

9. 92 2. 2014.332 .321883 6. 23 2. 0013.132 .101884 6. 082.0512 .201 .961884

Among the varieties which were analyzed in 1884 , and also previously, six have shown a tendency to continued degeneration in their percentages of nitrogen and size. The rest have shown signs of im. provement or remained stationary. The changes, then, which have been observed from year to year must be attributed to season and not to 
the soil, although continued cropping on one soil, even with fertilizers, appears from the experiments of Lawes and Gilbert to somewhat diminish the percentage of nitrogen. Another year's crop will furnish interesting data upon this subject, no doubt coufirming the views of the experimenters just mentioned that season has a greater effect upon grain than any other condition.

Among these analyses are found samples of wheat which have the greatest weight per bushel and per hundred grains of any which have been examined. These extremes are not, however, coincident, as may be seen from the following figures:

Extremes among Colorado wheats of 1884.

\begin{tabular}{ll|l|r|r|r}
\hline & & & \\
\hline
\end{tabular}

The weight per bushel is dependent on various causes. High weight is almost, if not always, an evidence of high quality, but not always of a large, plump, well-ma ured grain. The hard red spring wheat of the Northirest, which is small in size, and not well matured in the sense of haring a plump berry, with its usual amount of starch, is very heavf in its weight per bushel, while the large full wheat of Oregon, which is rery starchy, is light in weight.

The following data show the variation:

Weight per bushel, $f \cdot c$, of hard, sofi, and immature whents.

HARD RED SPRING WHEAT.

\begin{tabular}{|c|c|c|c|c|c|c|}
\hline No. & State. & $\begin{array}{l}\text { Weight } \\
\text { per } \\
\text { bushel. }\end{array}$ & $\begin{array}{l}\text { Weight } \\
\text { per } 100 \\
\text { grains. }\end{array}$ & Yield. & Quality. & $\begin{array}{l}\text { Albumi- } \\
\text { noids. }\end{array}$ \\
\hline $\begin{array}{l}1868 \\
1864 \\
1865 \\
1866 \\
1867 \\
1868 \\
1869 \\
2109 \\
2111 \\
1644 \\
2107\end{array}$ & 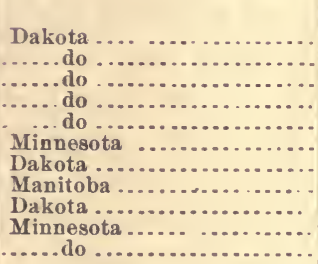 & \begin{tabular}{|r|} 
Pounds. \\
65.3 \\
66.5 \\
66.2 \\
65.2 \\
65.2 \\
65.5 \\
66.8 \\
67.1 \\
63.4 \\
64.9 \\
64.3
\end{tabular} & $\begin{array}{r}\text { Grams. } \\
3.312 \\
2.802 \\
3.368 \\
3.389 \\
2.921 \\
2.780 \\
3.700 \\
3.465 \\
3.074 \\
3.331 \\
2.926\end{array}$ & $\begin{array}{c}\text { Bush. } \\
25 \frac{1}{2} \\
26 \frac{1}{2} \\
27 \\
27 \frac{1}{4} \\
36 \\
(?) \\
(?) \\
(?) \\
(?) \\
(?) \\
(?)\end{array}$ & 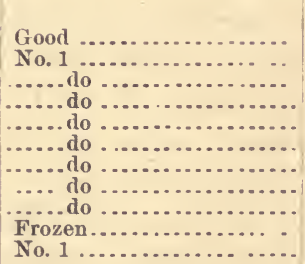 & $\begin{array}{r}\text { Per cent. } \\
14.53 \\
15.23 \\
17.33 \\
14.00 \\
14.35 \\
16.35 \\
16.28 \\
13.48 \\
18.03 \\
13.65 \\
13.83\end{array}$ \\
\hline
\end{tabular}

SOFT WHITE OREGON IVHEATS.

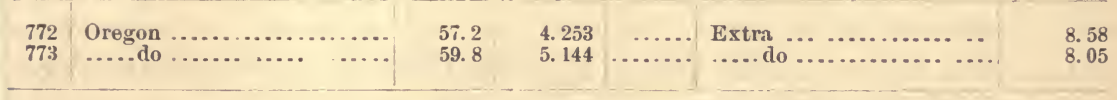


Weight per bushel, \&c., of hard, soft, and immature wheat-Continued.

OTHER SOFT WHEATS.

\begin{tabular}{|c|c|c|c|c|c|c|}
\hline No. & State. & $\begin{array}{c}\text { Weight } \\
\text { per } \\
\text { bushel. }\end{array}$ & $\begin{array}{l}\text { Weight } \\
\text { per } 100 \\
\text { grains. }\end{array}$ & Yield. & Quality. & $\begin{array}{l}\text { Albumi- } \\
\text { noids. }\end{array}$ \\
\hline 832 & Penusvlrania ............... & $\begin{array}{c}\text { Pounds. } \\
60.4\end{array}$ & $\begin{array}{r}\text { Grams. } \\
2.710\end{array}$ & $\begin{array}{c}\text { Bush. } \\
44\end{array}$ & Ordinary ................ & $\begin{array}{r}\text { 'Per cent. } \\
9.98\end{array}$ \\
\hline 759 & 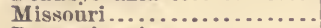 & 62.7 & 3. 860 & $\ldots \ldots \ldots$ & 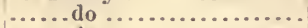 & 11. 19 \\
\hline 1288 & 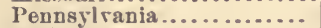 & 62.1 & 2. 526 & $\ldots . .$. & ..... do ....................... & 10.50 \\
\hline 1293 & 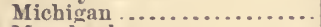 & 62.1 & 4. 196 & $\ldots \ldots$ & 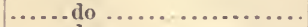 & 10.85 \\
\hline 1355 & Maryland................... & 63. 4 & 3.077 & $\ldots \ldots$ & 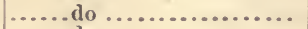 & 10.85 \\
\hline 1356 & North Carolina ............. & 66.2 & 3.653 & ....... & 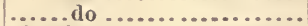 & 10.55 \\
\hline 1853 & West Virginia ............. & 64.5 & 3. 392 & 15 & 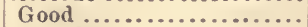 & 11. 30 \\
\hline 2112 & 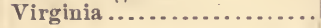 & 65. 0 & 3. 569 & 20 & 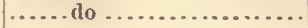 & 12. 60 \\
\hline
\end{tabular}

IMMATURE AND POOR WHEATS.

\begin{tabular}{|c|c|c|c|c|c|c|}
\hline 1804 & Alabama............. & 52. 3 & 2.011 & 3.5 & 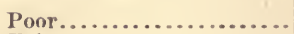 & 10.85 \\
\hline 1305 & 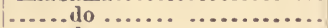 & 62.3 & 3. 710 & 10.3 & 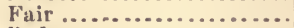 & 10.85 \\
\hline 1806 & $\ldots \ldots$ do............. & 49. 8 & 2.242 & 5.2 & 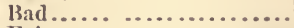 & 9.98 \\
\hline 1809 & ...... do ........... & 63.5 & 3. 486 & 5.3 & 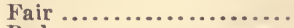 & 11.03 \\
\hline 1812 & 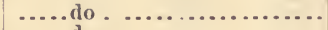 & 48.1 & 2. 166 & 2.8 & 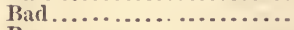 & 9.80 \\
\hline 1813 & ..... do ......................... & 57.0 & 2. 675 & 1.6 & 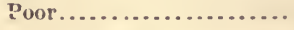 & 11.38 \\
\hline
\end{tabular}

AVERAGE OF 42 POOR WHEATS FROM OHIO IN 1883.

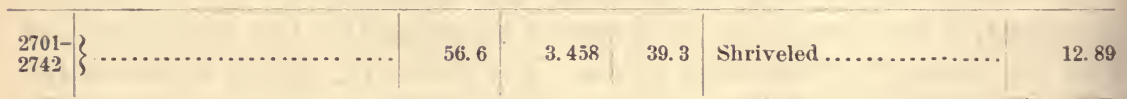

WHEATS WITH HIGHEST AND LOWEST ALBUMEN AND LARGEST SIZE.

\begin{tabular}{|c|c|c|c|c|c|c|}
\hline 2111 & 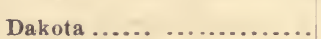 & 63.4 & 3.074 & & High albumen.... & 18.03 \\
\hline 1854 & Washingtun Territory..... & 63.5 & 2. 584 & ........ & 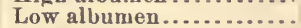 & 7.70 \\
\hline 3536 & 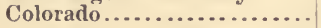 & 64.4 & 5.560 & ....... & ....................... & 12.08 \\
\hline
\end{tabular}

HIGHEST WEIGHT PER BUSHEL.

\begin{tabular}{|c|c|c|c|c|c|c|}
\hline 3570 & Colorado.................... & 68.6 & 4.060 & $\ldots \ldots$ & Hard red .......... & 12.95 \\
\hline
\end{tabular}

LOWEST WEIGHT PER BUSHEL.

\begin{tabular}{|c|c|c|c|c|c|}
\hline 1812 & Alabama ................... & 48 & 2. 165 & Immature $\ldots \ldots \ldots \ldots \ldots$ & 9.80 \\
\hline
\end{tabular}

From these figures, which have been obtained by weighing miniature bushels which were gradnated by comparison with the weight of large amounts of grain in struck bushels, it appears that hard spring wheat will average about $65_{\frac{1}{2}}^{1}$ pounds per pushel, soft white Oregon $58 \frac{1}{2}$ pounds, the ordinary soft wheat of the East 62.5, the poorly-matured grain of Alabama 55.5, the crop of 1883 in Ohio 56.6, while we have seen that the large plump Colorado grain weighs 65.2 pounds. The averages for different seasons in Coloradio vary directly as the percentages of albuminoids, although among the less fully-inatured grain the lighter often contains more uitrogen from lack of starch, as in the case of the Ohio crop of 1883. This was found to be the case by Lawes and Gilbert in their experiments, but does not always hold trae, as may be seen among the Alabama wheats and some others. The Oregon wheats finely matured, rich in starch, and low in nitrogen, which are very spongy and light in weight, are illustrative of this point. 
Conditions of growth and seed formation are so many and so raried that what may be true for one locality will often not apply to another which is far distant.

\section{RELIABILITY OF SPECIMENS AND SAMPLING.}

The question has been raised as to whether any specimen or sample of wheat rould represent the arerage composition of a field or large crop, or of a large mass of wheat in elevator, for instance, and whether the analyses of the specimens which hare been examined in this and previous reports could be relied on on this account.

An attempt has been made to solve this question, and with satisfactory results. In Bulletin No. 1 of this dirision analyses are given of two samples of wheat from the same lot of grain purchased by the Department as seed, the one selected in 1881 and the other in 1882, and analyzed without it being known to any one that they were intended to be identical. The results were closer than would probably be the case in most sampling.

Red Mediterranean wheat.

\begin{tabular}{|c|c|c|}
\hline & 1882. & 1883. \\
\hline 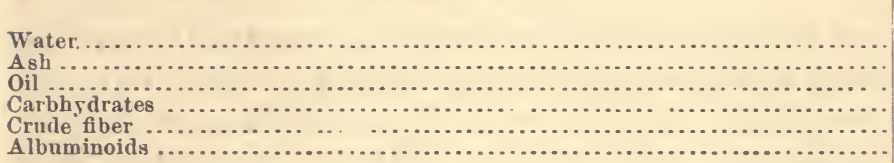 & \begin{tabular}{|r} 
Per cent. \\
9.83 \\
1.70 \\
2.21 \\
73.73 \\
1.68 \\
10.85
\end{tabular} & $\begin{array}{r}\text { Per cent. } \\
9.88 \\
1.62 \\
2.06 \\
73.80 \\
1.79 \\
10.85\end{array}$ \\
\hline
\end{tabular}

To decide as to rariations in composition in different parts of the same field and of the same farm, and of different varieties on the same farm and of differently developed heads and sized kernels of the same rariety, personal selections were made from a wheat farm in Carroll County, Maryland, belonging to Mr. Alastair P. Gordon-Cumming.

The specimens may be described as follows, with a determination of albuminoids-a point which, it was beliered, will settle any large variation in composition :

From a field of Fultz, bottom land, best quality.

\begin{tabular}{|c|c|c|c|c|c|c|c|c|c|}
\hline & 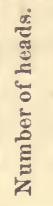 & 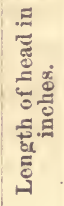 & 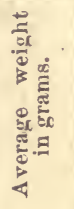 & 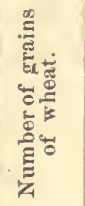 & 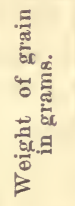 & 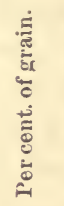 & 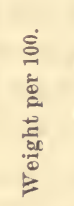 & 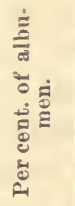 & Name. \\
\hline 2769 & 21 & $\ldots$. & 0.80 & 325 & 14.67 & 79.3 & 4. 517 & 9. 80 & Rakings. \\
\hline
\end{tabular}

From twenty-two arerage heads were selected:

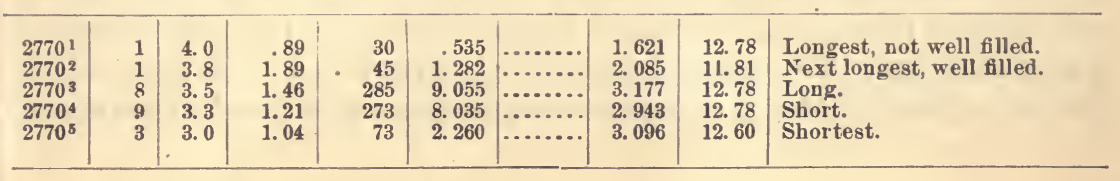


A patch of white Mediterranean seed from Department, second year's growth.

Serial No. 2768, 33 heads, weighing 37.5 grams and yielding 567 grains, or 75.2 per cent., weighing 28.197 grams, of which 461 were sound and 106 sprouted in the stack.

The sound weighed per $100,4.875$ grams; the sprouted, 5.400 , and contained albuminoids; the sound, 12.08 per cent.; the spronted, 13.48.

A field of Fultz, from a different portion of the same farm, gave among 14 average heads, weighing 391 grains:

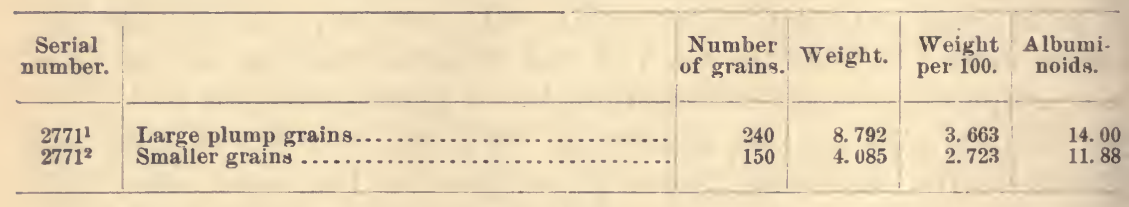

Among the Fultz sports were found:

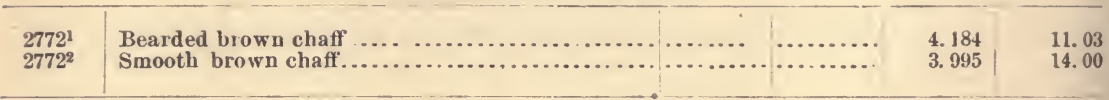

The results show that where the divisions have been made on marked characteristics there is a difference in composition; but that for arerages from the same field, even where some physical differences could be noted, there is little variation on analysis. Three of six samples from a field of Fultz were exactly alike in their percentage of albuminoids, another was only .18 per cent. different, or practically the same, while the rakings from the field were, as might be expected, low in albuminoids. One selected head also fell below the average composition for some unexplained reason. In this field of Fultz, therefore, there seems to be sufficient evidence to give us confidence in our results.

The next samples were taken from a shock of wheat, and the grain was found on preparation for analysis to have sprouted. It was therefore divided into two parts. The grain which sprouted was the heavier and best developed and contained the most albuminoids. This is also the case in another field of Fultz on the same farm, where an average sample was divided into large and small grains, the larger having the most albuminoids. That this is not by any means al ways the case, how. ever, it seems fair to believe from our experience with high relative proportion of nitrogen in specimens of shriveled wheat from other parts of the country. These kernels were none of them shrivelled; on the contrary, plump, and that some were merely more vigorous than others must stand as an explanation.

Sports or stragglers in the same field of Fultz were found to vary very largely from each other and a little more than the different-sized graius of Fult\%. This must be attributed to peculiarities in the variety and their different ability to assimilate nitrogen under the circumstances. 
These remarks must be regarded as suggestious only, as any absolute interpretation of the results is impossible. They serve, however, to show the constancy of the average composition of an average head, and of the average of the crop.

\section{CHARACTERISTICS OF 'IHE WHEA'T GRAIN.}

From observations in this and previous reports it may be said that of all grain wheat is probably the most susceptible to its enviroument.

Oats in certain directions are more variable, but in their general char. acteristics are much more permanent, as will appear in subsequent pages. The inherent tendency to change which is found in all grains is most prominent in wheat. It may be fostered by selection and by modifying such of the conditions of environment as it is in the power of man to affect.

The most powerful element to contend with is the character of the season or unfarorable climatic conditions. The injury done in this way is well illustrated in Colorado, and it would seem advisable in such cases to seek seed from a source where everything had been favorable, and begin selection again.

It must beborne in mind that selection must be kept up continuously, and that reversion takes place more easily than improvement. It took but one season to seriously injure Professor Blount's wheats, but it will be two or more years before they have recovered from that injury. Hallett in England was able to make his celebrated pedigree wheat hy selection, carried on through many years, but the same wheat grown by the ordinary farmer uxder unfarorable conditions for a few years without care has reverted to an ordinary sort of grain.

The effect of climate is well illustrated by four specimens of wheat which are to be seen in the collection of the chemical division. Two of these were from Oregon and Dakota some years ago, and present the most extreme contrast which can be found in this variable grain. One is light yellow, plump, and starchy, and shows on analysis a very small proportion of albuminoisls; the other is one of the sinall, hard, and darkcolored spring wheats of Dakota, which are rich in albuminoids. Between these staud two specimens from Colorado, which have been raised from seed similar to the Oregon and Dakota wheat. They are scarcely distinguishable except by a slight difference in color. The Colorado climate is such as to have modified these two seed wheats, until after a few years' growth they are hardly distinguishable in the kernel.

All localities having widely different climates, soils, or other conditions produce their peculiar rarieties and inodify those which are brought to them.

The result of these tendencies to change and reversion from lack of care in seed selection or other case has led to the practice of change of seed among farmers. A source is sought where either through greater care or more farorable conditions the variety desired has been able to 
hold its own. Sometimes this change is rendered necessary by conditions which are beyond the power of man to modify. As an example, No. 10 of Professor Blount's wheats, known as "Uregon Club," a white rariety from Oregon, has been deteriorating every year since it has been grown in Colorado, whereas if the seed had been supplied erery season directly from Oregon the quality would probably have remained the same. In extension of this illustration, the fact may be mentioned that annual renewal of the seed from a desirable and favorable source often makes it, possible to raise cereals where otherwise the climatic conditions would render their cultivation impossible through rapid reversion. This is particularly the case with extremes in latitude, the effect of which is found not so much upon the composition of the crop as ou the yield and size of the grain. In the South the warmer climate, together, of course, with poorer soil and cultivation in many instances, reduces the sield. The average in different States is given by Mr. Dodge as follows:

Yield per acre of wheat.

\begin{tabular}{|c|c|c|c|c|c|}
\hline State. & $\begin{array}{c}\text { Per } \\
\text { cent. }\end{array}$ & State. & $\begin{array}{c}\text { Per } \\
\text { cent. }\end{array}$ & State. & $\begin{array}{l}\text { Per } \\
\text { cent. }\end{array}$ \\
\hline Maine & 15. 2 & South Carolina. & 5.6 & Michigan & 19.5 \\
\hline New Hampshire & 15.0 & Georgia ....... & 6.6 & Indiana... & 18.0 \\
\hline Vermont .......... & 16.3 & Florida .... & 5. 2 & Illinois & 15. 9 \\
\hline Massachusetts & 16.4 & Alabama & $5 . \overline{7}$ & Wisconsin & 12.8 \\
\hline Rhode Island. & 14.1 & Mississippi ...... & 5.0 & Minnesota .... & 11.4 \\
\hline Connecticnt.. & 17.6 & Nevadit....... & 16. \& & Iowa. & 10.2 \\
\hline New York... & 15. 7 & Louisiana................. & 3.4 & Missouri.................. & 12.0 \\
\hline New Jersey. & 12.7 & Texas .......... & 6.8 & Kansas .... & 9.3 \\
\hline Pennsylvania .... & 13.5 & A rkansas ................. & 6.2 & Nebraska.... & 9.4 \\
\hline Delaware .... & 13.4 & Tennessee .......... & 6.1 & California. & 22.0 \\
\hline Maryland. & 14.1 & West Virginia & 10.2 & Oregon & 15.8 \\
\hline Virginia...... & 8.7 & Kentucky & 9.8 & Colorado................ & 18.9 \\
\hline North Carolina. & 5.2 & Ohio ...................... & 18.0 & & \\
\hline
\end{tabular}

It must be remembered, however, that three quarters of the wheat crop has beeu produced in the ten States of Illinois, Indiana, Ohio, Michigan, Minnesota, Iowa, California, Missouri, Wisconsin, and Pennsylrania, and that a State like Minnesota unust not be considered as furnishing the largest part of the supply merely because wheat is the prevailing crop in that State.

Having shown the conditions under which unfarorable and farorable variations occur, it is hoped that these investigations will be the means of aiding those who are engaged in the improrement of the yield and quality of the crop of our country.

It seems proper in this place to allude also to the immense amount of the best food elements of our soils which are yearly being taken from the farm and exported from our ports in the shape of nitrogen and the mineral constituents of the grain, the loss of which is continually ren. dering our wheat lands poorer and forcing the cultivation into new parts of the country where the soil is still of virgin richness. If grain is sold off the farm the loss of nitrogen and minerals must be replaced 
by fertilizers. In the East this is already done, but in the West it seems that nothing but experience of wasting fertility will teach the lessoll.

\section{OATS.}

Oats, the third in importance of our cereal crops, as far as production is concerned, are grown under as varied conditions as auy of them and are as a crop the most variable in their appearance.

They will grow and can be made to pay on almost any soil, and, although flourishing in cold climates, can be successfully raised in the far South by sowing as a winter grain and so reaching maturity in the cool part of the year.

In consequence of these variations in the conditions of growth there are to be found the greatest differences in weight and size of the grain, its plumpness, and the relative proportion of kernel and husk. Dependent on these differences many classifications have been proposed, but as there seems to be a regular gradation from the one extreme of the white potato oats to the other of red rust proof, there seems to be no definite basis for varieties due to color, shape of the grain, or plumpness. Botanically, there seem to be three varieties recognized-the common Avena sativa L., and two others, A. orientals Schb., haring the kernels all on one side of the stem, and $A$. nuda L., to the grain of which the husks or chaff are not arlherent.

For our consideration, from a chemical and physical point of view, these distinctions are of small importance, as the same variations are found among each species.

To the farmer the most important characteristic, and the one by which this grain is usually valued, is its weight per bushel. In close relation to this is the proportion of keruel to husk-a point which has been little, if at all, investigated. In the specimens which have been collected from the most prominent regions where oats are grown these characteristics have been determined, as well as the size aud weight of the kernels, with the purpose of studying not only the way in which they are affected by surrounding causes, but also their relation to the composition of the grain.

The sources of the specimens examined, their color and shape, and other physical characteristics are here presented. 


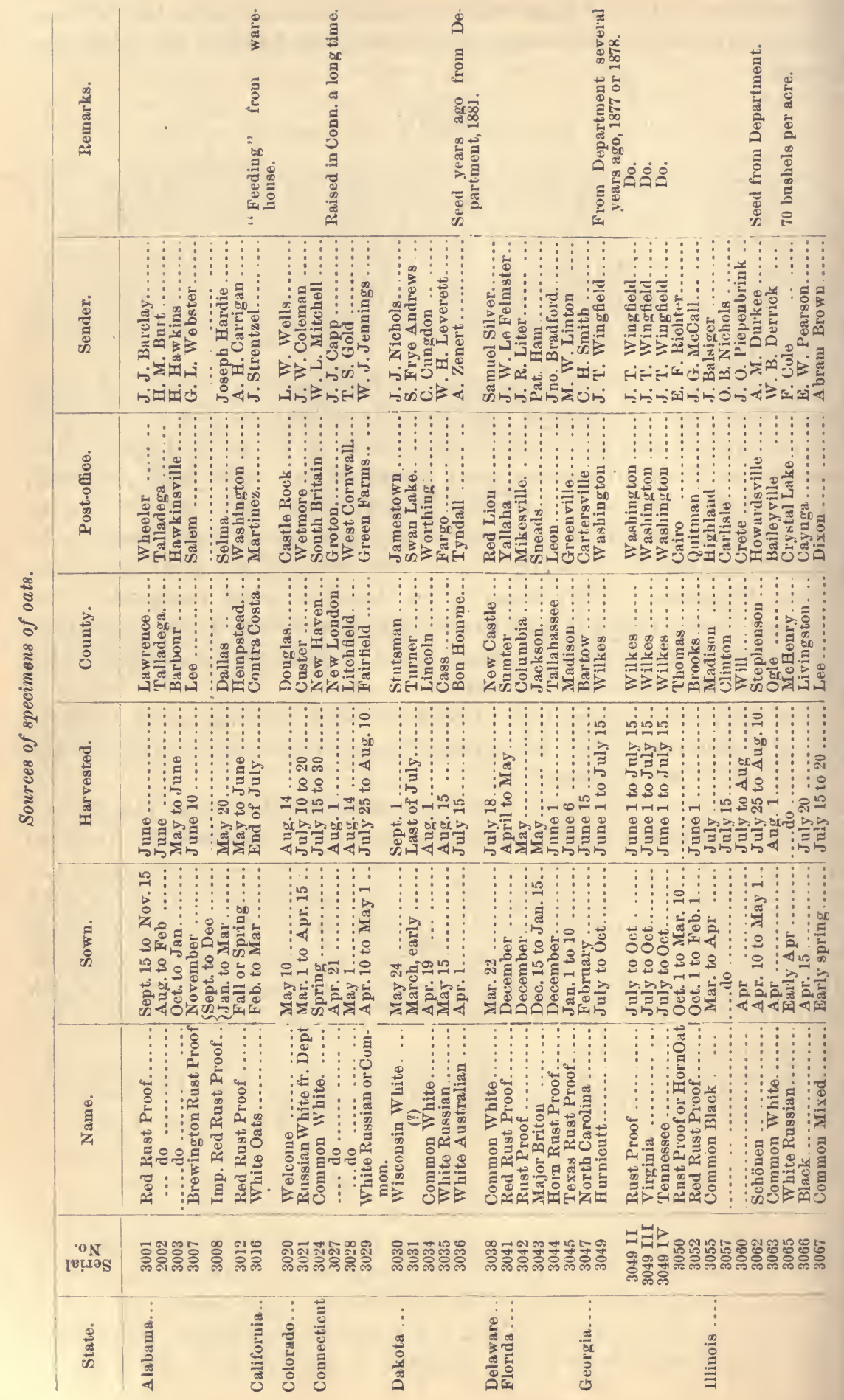




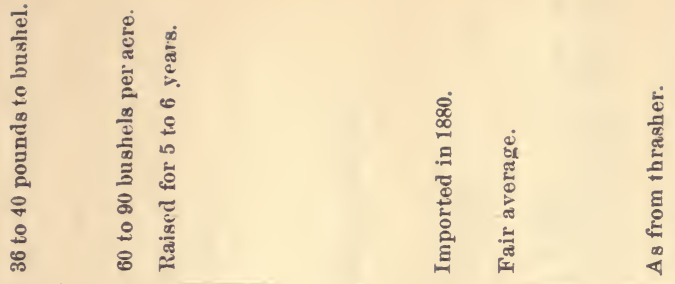

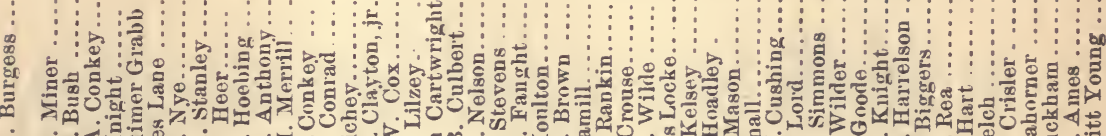

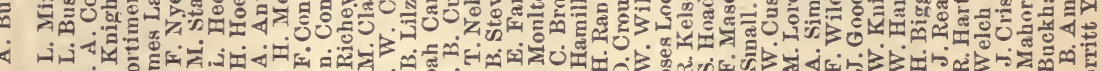

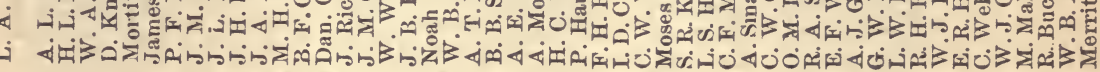

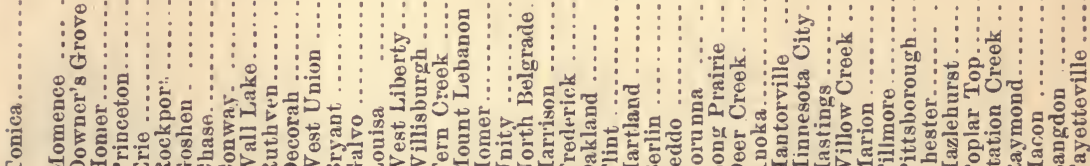
ह

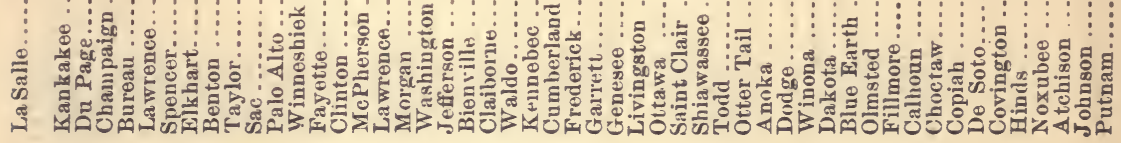

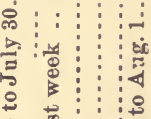

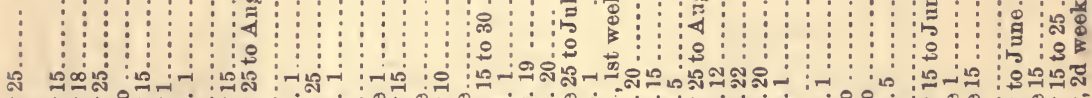

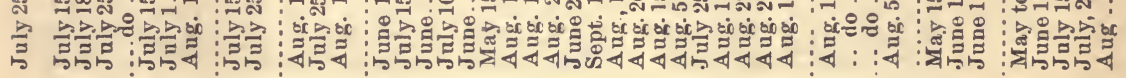

(1)

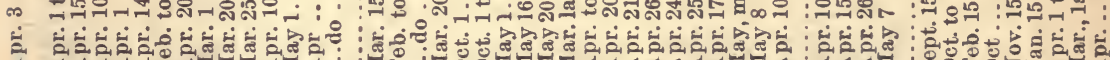

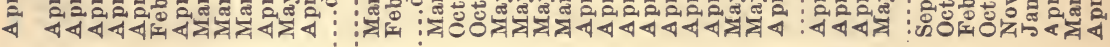

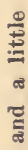

五

过

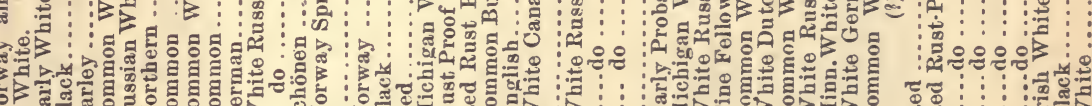
冬

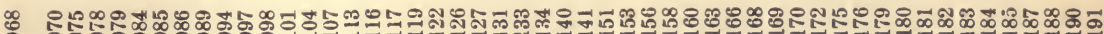
ठำ

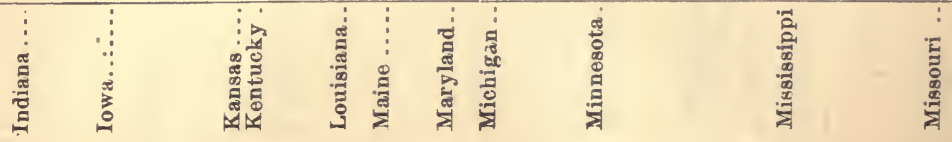




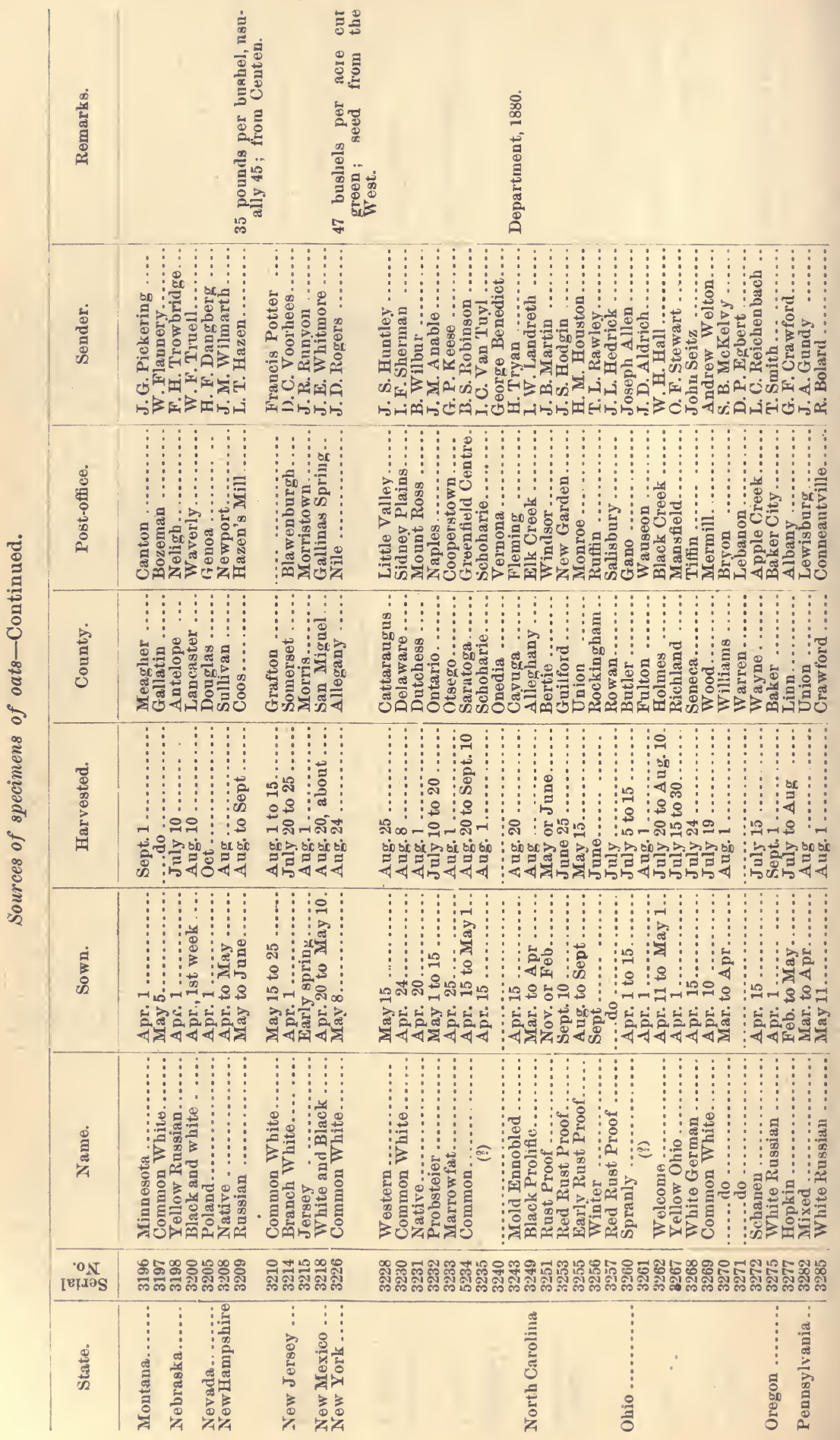




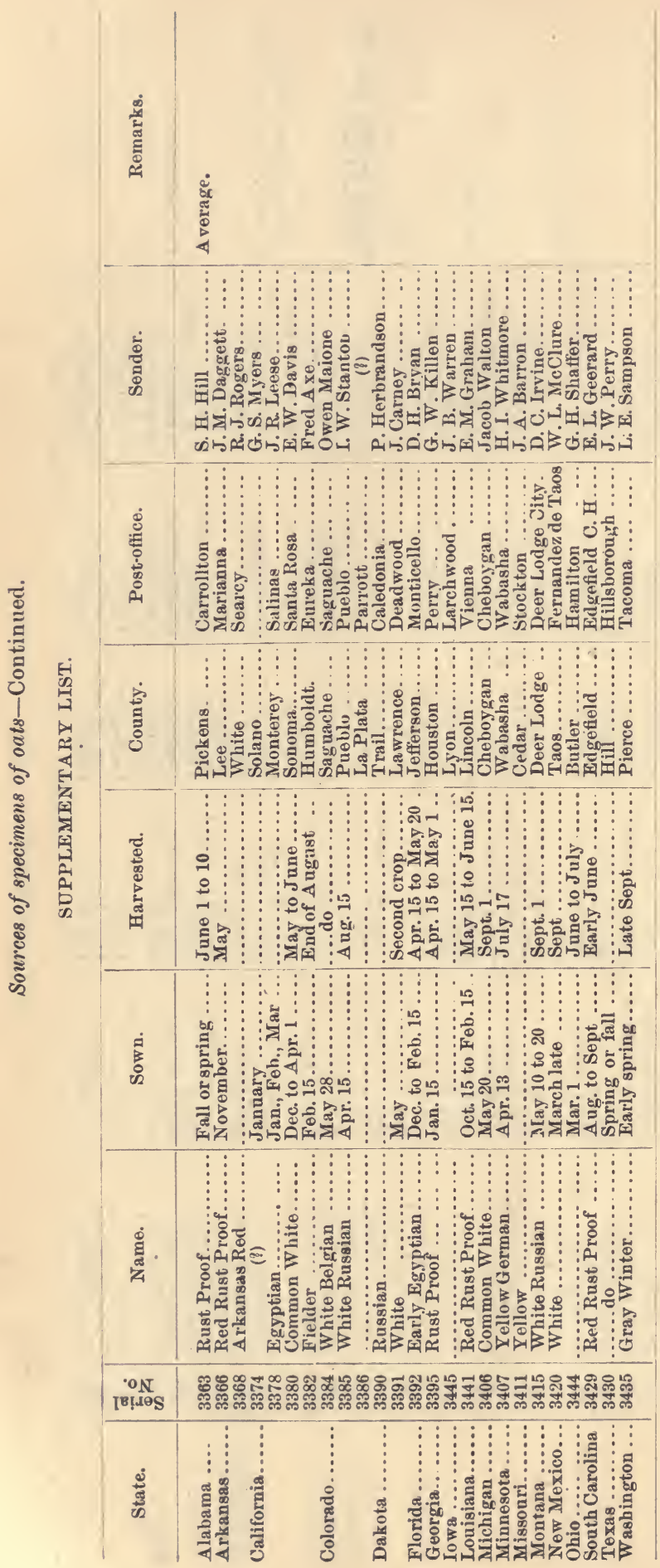


Physical properties of oats.

\begin{tabular}{|c|c|c|c|c|c|c|c|}
\hline State. & $\begin{array}{c}\text { Serial } \\
\text { num. } \\
\text { ber. }\end{array}$ & $\begin{array}{l}\text { Weisht } \\
\text { of loo } \\
\text { grains. }\end{array}$ & $\begin{array}{l}\text { Clean } \\
\text { grain. }\end{array}$ & $\begin{array}{l}\text { Hulled } \\
\text { grain. }\end{array}$ & Hulls. & $\begin{array}{c}\text { Weight } \\
\text { per } \\
\text { bushel. }\end{array}$ & Color. \\
\hline & & Grams. & Percent. & Per cent. & Per cent. & $L b s$. & \\
\hline Maine. & 3131 & $2.1+4$ & 913.02 & 69.54 & 30.46 & 34. 7 & Light. \\
\hline Do .... & 3133 & 2. 6111 & 98. 22 & 69. 6.5 & 3035 & 44. 2 & White. \\
\hline Do $\ldots \ldots$. & 3134 & 2. 279 & 97.89 & 71.68 & 28.32 & 315.8 & Light. \\
\hline Now Hampshire . ..... & 32118 & 2. 0150 & $98 \quad 104$ & $\cdots \cdots$ & & 33.5 & Yollow and green. \\
\hline Do ................. & 32,19 & 2. 890 & 8.5. 34 & 70.88 & 29. 12 & 40.7 & Ypllow. \\
\hline$D_{0} \ldots \ldots \ldots \ldots \ldots$ & 3210 & 2. 465 & 98.111 & 72. 20 & 27.80 & 41). 7 & I.ight. \\
\hline Vermont.............. & $3 ? \div 2$ & 2. 3.24 & 87. 27 & 71.01 & 28.99 & 411. 2 & WV bite. \\
\hline Do $\ldots \ldots \ldots \ldots \ldots$ & 3323 & 2. 122 & 93.74 & 711. 98 & 29.02 & 36.9 & Light. \\
\hline Do $\ldots \ldots \ldots \ldots$ & 3324 & 2.695 & 93. $6 . \overline{3}$ & 7139 & 28.61 & 37.3 & Do. \\
\hline Do $\ldots \ldots \ldots$ & $33: 6$ & 2. 756 & 95.22 & 64.72 & 3... 28 & 43.1 & 1). \\
\hline Connecticnt ........... & 3024 & 1. 796 & 97. $0 ! 3$ & 69.2 .5 & 30.7 .5 & 37. 7 & Yellıw aud green. \\
\hline Do $\ldots . . . . . . .$. & $30: 27$ & 1. 93.5 & 91.90 & 6.50 & 31.30 & 29.5 & Light. \\
\hline Do & 3.) 8 & 2. 128 & 97.47 & 72.18 & 27.82 & 37.6 & White. \\
\hline Do $\ldots . . . . . .$. & 3029 & 2. 580 & 96.15 & 62.63 & 37.37 & 37.6 & Light brown. \\
\hline Total. & ......... & 8.439 & $3 \times 3.11$ & 26!. 76 & $13^{11}, 24$ & 142.4 & \\
\hline Arerage.... & $\ldots \ldots$ & 2.810 & $9 ; .7 \times$ & 67.44 & 32.56 & 35.6 & \\
\hline New York....... & 3226 & 2. 342 & 98.70 & 66.70 & 33.30 & 39. 0 & Light. \\
\hline Do $\ldots . .$. & $3 \geq 8$ & 2.663 & 9.2 .57 & 73. 83 & 26.17 & 40.1 & Mixed. \\
\hline Do.... & 3231) & 2.192 & 938 & & 31.80 & $\begin{array}{l}38.0 \\
33.2\end{array}$ & Light. \\
\hline$\left.D_{0}\right) \ldots . .$. & 3231 & $\begin{array}{l}2.192 \\
2.856\end{array}$ & $\begin{array}{l}9.7 .80 \\
96.10\end{array}$ & $\begin{array}{l}68.20 \\
73.50\end{array}$ & $\begin{array}{l}31.80 \\
26.5 t)\end{array}$ & 33. 2 & Wo. \\
\hline & 3232 & $\begin{array}{l}2.8 .56 \\
3.1: 7\end{array}$ & $\begin{array}{l}95.111 \\
98.17\end{array}$ & 71.49 & $\begin{array}{l}26.211 \\
2 \times .51\end{array}$ & $\begin{array}{l}42.9 \\
41.8\end{array}$ & Do. \\
\hline 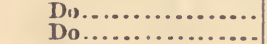 & 3233 & $\begin{array}{l}\text { 3. } 1: 7 \\
\text { 2. } 1399\end{array}$ & 99.14 & 711.82 & 29.18 & & Do. \\
\hline 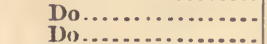 & 3234 & $\begin{array}{l}\text { 2. } 039 \\
2.921\end{array}$ & 8930 & 73.24 & 26.76 & $\begin{array}{l}37.3 \\
44.1\end{array}$ & White. \\
\hline $\begin{array}{l}\text { Do } D_{1} \ldots \ldots \ldots \ldots \\
D_{0} \ldots \ldots \ldots \ldots \ldots\end{array}$ & 32.35 & $\begin{array}{l}\text { 2. } 921 \\
2.430\end{array}$ & $9 \div \times 1$ & 710.20 & 29.80 & $\begin{array}{l}44.1 \\
41.9\end{array}$ & Brown. \\
\hline $\begin{array}{l}\text { Do............... } \\
\text { New Jersey....... }\end{array}$ & $3: 43$ & $\begin{array}{l}\text { 2. } 430 \\
2.709\end{array}$ & 98.80 & 75.210 & & $\begin{array}{l}41.9 \\
41.4\end{array}$ & Io. \\
\hline $\begin{array}{c}\text { New Jersey ....... } \\
\text { Do ........... }\end{array}$ & 3214 & $\begin{array}{l}\text { 2. } 709 \\
2.033\end{array}$ & $\begin{array}{l}98.80 \\
98.20\end{array}$ & $\begin{array}{l}75.10 \\
70.40\end{array}$ & 24.90 & 41.4 & White. \\
\hline & $3 \geq 82$ & 2.207 & $9 r_{0} .4$ & 69.65 & 30.35 & 325 & 1)o. \\
\hline Do & 328.5 & 2.617 & 9694 & 69.114 & 311. .96 & 38.1 & Di). \\
\hline 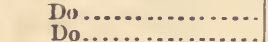 & $32 \times 6$ & 3.010 & $9+15$ & 71.34 & 28.66 & 43. 7 & WVhite. \\
\hline Do................ & $3: 86^{1}$ & 2910 & 81.44 & 64.18 & 35. $x^{2}$ & 37. $\mathrm{x}$ & Lisht. \\
\hline Ohio $\ldots . . . . . . . . .$. & 3260 & 2. 248 & 99. 311 & 7333 & 2667 & 39.9 & White. \\
\hline 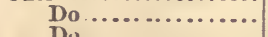 & $3 \div 61$ & 2. 130 & $87.7 t$ & $7+93$ & 25.05 & 41.0 & Liplit. \\
\hline$\left.D_{1}\right) \ldots \ldots \ldots . . . . .$. & 3263 & 2. 670 & $968 t$ & 60. 83 & 39.17 & 40.0 & Do. \\
\hline 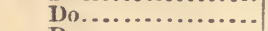 & 32157 & $27: 2$ & 95.31 & 72. 07 & 2793 & 39.9 & Wo. \\
\hline Do................ & 3268 & 2. 224 & $9 \times .30$ & 74.62 & 25.38 & 40.0 & Uo. \\
\hline Do.............. & $3: 69$ & 2. 010 & $9 \div 56$ & $6 ! 1.03$ & 30.92 & 37.8 & Yellow and green. \\
\hline Do ............... & 3270 & 2. 260 & 99.21 & 73. $\therefore 4$ & 26.46 & 40.1 & Light. \\
\hline Do ........ & $327 i$ & 2.012 & 99. 15 & 73. 31 & 26.69 & ...... & \\
\hline Do ................ & $34+t$ & 2.025 & 96.36 & 71.25 & 28.75 & ...... & \\
\hline Michiıan......... & 31.51 & 2. 4.10 & 98.59 & 74.30 & 2.70 & 41.1 & Light. \\
\hline$D_{1}{ }_{10} \ldots \ldots \ldots \ldots \ldots$ & 3153 & 2.4110 & 9 i. 2.3 & 72.16 & 27.84 & 43.6 & IV live. \\
\hline 1) & 31.6 & $2.86 \mathrm{t}$ & 97.58 & $7: .41$ & 27.59 & 42.8 & Light. \\
\hline Do.................. & 31.58 & 2.914 & $97.0 ! 1$ & 70.91 & 29.09 & 43. 7 & IVhite. \\
\hline Do ............ & 310 & 2.507 & $93.5 \times$ & 72.47 & 27.53 & 39.4 & Liglit. \\
\hline $110 . . . . . \ldots \ldots \ldots$ & 3406 & 3. $23 i$ & 94.2 .5 & 71.62 & 28. 38 & 43.2 & IV hite. \\
\hline Indiana ........... & 3084 & 2.414 & (9.6.) & $71 \% .69$ & 29. 31 & 35. 3 & Do. \\
\hline Do.................... & 31186 & 2. 1186 & $8 \cdot 7: 3$ & 73.40 & 215.60 & 39.4 & Lìnt. \\
\hline Do ............ & $30<9$ & 2.060 & 97. 71 & 71. 92 & 28.18 & 35.4 & Do. \\
\hline mlinois ............ & 31155 & & 96. 37 & $7+.75$ & 25.25 & 40.7 & Mixell. \\
\hline 1)o.......... & 30650 & 2.370 & & 66. 58 & 33.42 & 41.5 & W' lite: \\
\hline Do................ & 3060 & 2. 1133$)$ & 9.. 52 & 69.53 & 30.47 & 36.2 & Liglit. \\
\hline Do............ & 3063 & $1.9 \times 10$ & 17. 89 & $7 \div .74$ & 27.26 & 37.1 & 1.iulit brown. \\
\hline Do $\ldots \ldots \ldots \ldots \ldots$ & 31165 & 2.517 & 98.27 & 70.97 & 29.03 & 38.6 & Livhr. \\
\hline 1)o............. & 3.)(it & 2.512 & $9: 3.30$ & 7.5. $\times 5$ & 24.15 & 40.5 & Bruwn. \\
\hline Do............... & 30167 & 2. 014 & $9 \times 35$ & 70.46 & 29.54 & 38.8 & Mixed. \\
\hline Do..................... & 3018 & 3. 025 & 9893 & 74.97 & $2 i .03$ & 41.5 & Do. \\
\hline Do................... & $3068^{2}$ & & 9.5. (19) & 72.32 & $27.6 x$ & & \\
\hline Wisconsin ........... & 3357 & 2.920 & 95.24 & $70.5: 3$ & 29.47 & 43.7 & Light. \\
\hline Do..................... & $33: 3$ & 2.450 & 96. 53 & $68 \div 9$ & 31.01 & 39.7 & Mixel. \\
\hline Do........... & 3357 & 2.377 & 97.10 & 70. 03 & 29.97 & 3ส. 9 & White. \\
\hline J)o............. & 33150 & 2. 580 & 8!). 6.3 & 73.25 & 26.75 & 35. 6 & 1)o. \\
\hline Do.............. & $33 t i t$. & 2.1109 & 9.5 .55 & 71.05 & 28.95 & 37.1 & 10\%. \\
\hline Minuesota ............ & 31 (i6 & 2.180 & 97.84 & 69.27 & 30.73 & 35.4 & Liglit brown. \\
\hline 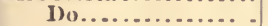 & $311 ; 8$ & 2. 038 & $9 \times .11$ & 73.50 & 26. 50 & 42.0 & White. \\
\hline Do................. & 3169 & 2. $6: 8$ & 99. 39 & 69.88 & 311.12 & 40.2 & Io. \\
\hline Do $\ldots \ldots \ldots \ldots \ldots$ & 3170 & 2.170 & 93.46 & 73. 00 & 27. 10 & 38.3 & Liglit. \\
\hline Do............ & 3172 & 2. 770 & & 72.62 & 27.38 & 48.4 & White. \\
\hline$\left.D_{1}\right) \ldots \ldots \ldots \ldots \ldots$ & 317.5 & 2. 1166 & 91.80 & 72.40 & 27.60 & 39.8 & Mixed. \\
\hline Do.............. & $3175^{2}$ & 2.075 & 93. 72 & 7.2 .91 & 27.09 & 38.1 & I)o. \\
\hline Do................. & 3176 & 2.610 & 98.14 & 69.60 & 3040 & 40.5 & White. \\
\hline Do .................. & 3179 & 2. 162 & 96.10 & 71.90 & $2 \times, 10$ & 39.6 & Light. \\
\hline owa...................... & 3094 & 1. 924 & 97. 53 & 67.31 & 32.69 & 33.5 & 1). \\
\hline Do................. & 3097 & 2.402 & 9i5. 5.5 & 73.43 & 26.57 & 40.6 & White. \\
\hline Do............. & 3098 & 2. 253 & 97. 17 & 74.78 & 25.22 & & \\
\hline Do......... & 3101 & 2.413 & 9.5. 94 & 71.87 & 28.13 & 42.1 & Mixed. \\
\hline Do.................... & $310 t$ & 2.172 & 97.16 & 711. 07 & 29.93 & $3 \times .9$ & Light. \\
\hline Do................... & 3107 & 2. 308 & 97.67 & 72.34 & 27.66 & 37.1 & Brown. \\
\hline
\end{tabular}


Physical properties of oats-Coutinued.

\begin{tabular}{|c|c|c|c|c|c|c|c|}
\hline State. & $\begin{array}{l}\text { Serial } \\
\text { num- } \\
\text { ber. }\end{array}$ & $\begin{array}{l}\text { Weight } \\
\text { of } 100 \\
\text { grains. }\end{array}$ & $\begin{array}{l}\text { Clean } \\
\text { grain. }\end{array}$ & $\begin{array}{l}\text { Hulled } \\
\text { grain. }\end{array}$ & Hulls. & $\begin{array}{c}\text { Weight } \\
\text { per } \\
\text { bushel. }\end{array}$ & Color: \\
\hline Missouri. & 3190 & $\begin{array}{l}\text { Grams. } \\
2.016\end{array}$ & $\begin{array}{r}\text { Per cent. } \\
97.84\end{array}$ & $\begin{array}{r}\text { Per cent. } \\
71.45\end{array}$ & $\begin{array}{r}\text { Percent. } \\
28.55\end{array}$ & $\begin{array}{r}\text { Pounds. } \\
36.7\end{array}$ & \\
\hline $\begin{array}{l}\text { Do.. } \\
\text { Do.. }\end{array}$ & 3191 & 1. 630 & 97.70 & 68.60 & 31.40 & $\begin{array}{r}36.7 \\
\ldots \ldots\end{array}$ & Mixed. \\
\hline $\begin{array}{r}\text { Do... } \\
\text { Nobraska.. }\end{array}$ & 3411 & 1.956 & 98.85 & 69. 28 & 30. 72 & 38.8 & Yellow. \\
\hline $\begin{array}{r}\text { Nobraska... } \\
\text { Do... }\end{array}$ & 3198 & 2.194 & 99.00 & 73.20 & 26. 80 & 39.5 & Do. \\
\hline $\begin{array}{l}\text { Do... } \\
\text { Do... }\end{array}$ & 3200 & $\begin{array}{l}1.582 \\
1.512\end{array}$ & 97.20 & 68.30 & 31.70 & 30.2 & Mixed. \\
\hline $\begin{array}{r}\text { Do.. } \\
\text { Dakota... }\end{array}$ & $\begin{array}{l}3200^{1} \\
3030\end{array}$ & & $\begin{array}{l}97.40 \\
96.43\end{array}$ & 68. 79 & 31.21 & 29.7 & Do. \\
\hline Dakota... & 3035 & $\begin{array}{l}\text { 2. } \\
2.367\end{array}$ & $\begin{array}{l}96.43 \\
92.04\end{array}$ & $\begin{array}{l}67.90 \\
72.39\end{array}$ & 32. 10 & 40. 2 & WVbite. \\
\hline Do... & 3036 & 2. 957 & 96.12 & $\begin{array}{l}72.39 \\
62.20\end{array}$ & 27. 61 & 44. 7 & Yellow. \\
\hline Do... & 3390 & 2.844 & 92.33 & $\begin{array}{l}62.20 \\
73.16\end{array}$ & 37.80 & 48. 6 & Light. \\
\hline Do... & 3391 & 2.372 & 87.70 & $\begin{array}{l}73.16 \\
55.37\end{array}$ & $\begin{array}{l}26.84 \\
44.63\end{array}$ & 44. 3 & Do. \\
\hline Montana... & 3196 & 2.528 & 94.49 & 70.10 & $\begin{array}{l}44.63 \\
29.90\end{array}$ & $\begin{array}{l}38.8 \\
45.0\end{array}$ & $\begin{array}{l}\text { White. } \\
\text { White and bro }\end{array}$ \\
\hline Do... & 3197 & 2.010 & 90.16 & 69.15 & 30.85 & $\begin{array}{l}45.0 \\
45.4\end{array}$ & $\begin{array}{l}\text { White an } \\
\text { White. }\end{array}$ \\
\hline $\begin{array}{l}\text { Do. . } \\
\text { Maryland . }\end{array}$ & 3415 & 2. 691 & 88.54 & 72.36 & 27.64 & 39.5 & $\begin{array}{l}\text { White. } \\
\text { Yellow. }\end{array}$ \\
\hline $\begin{array}{c}\text { Maryland . } \\
\text { Do.. }\end{array}$ & 3140 & 1.850 & 94.86 & 71.70 & 28. 30 & 35.0 & Ligbt. \\
\hline $\begin{array}{c}\text { Do.. } \\
\text { Delaware.. }\end{array}$ & 3141 & 2.637 & 91.65 & 71.36 & 28.64 & 40.0 & Do. \\
\hline $\begin{array}{l}\text { Delaware.. } \\
\text { Virginia... }\end{array}$ & $\begin{array}{r}3038 \\
3331\end{array}$ & 1.976 & 98. 04 & 69.59 & 30.41 & & \\
\hline $\begin{array}{r}\text { Virginia... } \\
\text { Do... }\end{array}$ & $\begin{array}{l}3331 \\
3334\end{array}$ & 2.720 & 93.00 & 72.40 & 27.60 & 39.0 & Brown. \\
\hline Do... & $\begin{array}{l}3334 \\
3335\end{array}$ & 2.771 & 96.92 & 59. 00 & 41.00 & 41.4 & White. \\
\hline Do... & $\begin{array}{l}3335 \\
3337\end{array}$ & 2.480 & 94.09 & 64.29 & 35.71 & $\begin{array}{l}35.5 \\
40.8\end{array}$ & $\begin{array}{l}\text { Light. } \\
\text { White. }\end{array}$ \\
\hline West Virgir & 3345 & 1.872 & 97.19 & 71.26 & 28.74 & $\begin{array}{l}40.8 \\
37.4\end{array}$ & $\begin{array}{c}\text { White. } \\
\text { Do. }\end{array}$ \\
\hline $\begin{array}{l}\text { Do... } \\
\text { Do... }\end{array}$ & 3346 & 3.386 , & 96.55 . & 67.59 & $\begin{array}{l}2 x .74 \\
32.41\end{array}$ & 38.3 & $\begin{array}{l}\text { Do. } \\
\text { Light. }\end{array}$ \\
\hline $\begin{array}{l}\text { Do... } \\
\text { J)o... }\end{array}$ & 3347 & 1.969 & 93.55 & 64.48 & 35. 52 & 38.5 & Do. \\
\hline $\begin{array}{l}\text { Jo } \\
\text { North Carolina... }\end{array}$ & 3348 & 2.773 & 96.45 & 62.60 & 37.40 & 36. 4 & Light brown. \\
\hline $\begin{array}{l}\text { North Carolina } \\
\text { Do........ }\end{array}$ & 3249 & 2.060 & 93.94 & 70.50 & 29.50 & 47.8 & Brown. \\
\hline 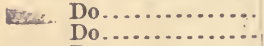 & 3251 & 1.834 & 97.10 & 70. & 29.70 & 36.4 & Mixed. \\
\hline $\begin{array}{l}\text { Do... } \\
\text { Do... }\end{array}$ & 3253 & 3. 362 & 94.30 & 68. & 31.30 & 37.2 & Brown. \\
\hline $\begin{array}{l}\text { Do... } \\
\text { Do... }\end{array}$ & 3255 & 2.470 & 88.84 & 70. & 29.56 & 39.5 & Black. \\
\hline Do... & $\begin{array}{l}3256 \\
3257\end{array}$ & $\begin{array}{l}2.314 \\
2.935\end{array}$ & 96.20 & 73. & 26. 66 & 41.1 & Light brown. \\
\hline South Carolina. & $\begin{array}{l}3257 \\
3295\end{array}$ & $\begin{array}{l}2.935 \\
3.039\end{array}$ & 96. 77 & $\begin{array}{l}68.95 \\
69.95\end{array}$ & 31.05 & 36.6 & Brown. \\
\hline Do..... & 3296 & $\begin{array}{l}3.039 \\
2.823\end{array}$ & $\begin{array}{l}98.51 \\
93.76\end{array}$ & $\begin{array}{l}69.95 \\
67.24\end{array}$ & 30.05 & $\begin{array}{l}39.5 \\
38.5\end{array}$ & $\begin{array}{l}\text { Do. } \\
\text { Do. }\end{array}$ \\
\hline Do... & 3297 & 2.055 & 96.22 & & 32.76 & $\begin{array}{l}38.5 \\
35.7\end{array}$ & $\begin{array}{l}\text { Do. } \\
\text { Light brown. }\end{array}$ \\
\hline Do... & 3298 & 2.052 & 96.90 & $\begin{array}{l}08.00 \\
68.72\end{array}$ & $\begin{array}{l}31.35 \\
31.28\end{array}$ & $\begin{array}{l}35.7 \\
35.9\end{array}$ & $\begin{array}{l}\text { Light brown. } \\
\text { Yellow. }\end{array}$ \\
\hline Do... & 3299 & 2. 831 & 94. 01 & 68.48 & $\begin{array}{l}31.28 \\
31.52\end{array}$ & & \\
\hline Do.. & 3300 & 3.176 & 97.59 & 71.20 & $\begin{array}{l}31.52 \\
28.80\end{array}$ & 41.5 & Brown. \\
\hline Do... & 3301 & 2.981 & 93.78 & 73.33 & 26.67 & 37.8 & Light brown. \\
\hline Do... & 3429 & 3.179 & 97.17 & 68. 61 & 31. 39 & 39.8 & Brown. \\
\hline Kentucks. & 3116 & 2.270 & 98. 52 & 72. 70 & 27.30 & & \\
\hline Do.... & 3117 & 1. 968 & 98.23 & 71. & 28.51 & 33. 3 & Mixed. \\
\hline Do.... & 3119 & 2. 897 & 92.22 & 68. & 31.49 & 81.9 & Brown. \\
\hline Do... & 3122 & 1.860 & 90.56 & 67. & 32.73 & 29.9 & Light. \\
\hline Tennessee.. & 3302 & 1.897 & 99.29 & 68.24 & 31.76 & 31.4 & Brown. \\
\hline Do & 3303 & 2.378 & 91.39 & 68. & 31.25 & 38.3 & Do. \\
\hline Do. & 3304 & 2. 920 & 97.11 & 67. 66 & 32.34 & 35.5 & Do. \\
\hline Do... & 3309 & 2.160 & 94.86 & 57. & 42.99 & 39.9 & Do. \\
\hline Georgia.... & 3047 & 2.236 & 98.44 & 70.95 & 29.05 & 33.4 & Light. \\
\hline Do... & 3049 & 3. 255 & 97.39 & 68.88 & 31.12 & n.......... & \\
\hline Do.. & $3049^{1}$ & 2.388 & 91.34 & 71.18 & 28.82 & ....... & \\
\hline Do.. & $3049^{2}$ & & 91.64 & 73. & 26.48 & 3 & \\
\hline Do.. & 3050 & 2. 609 & 96.42 & 65. & 34. 83 & 31.4 & Brown. \\
\hline $\begin{array}{l}\text { Do... } \\
\text { Do.. }\end{array}$ & 3052 & 2.830 & 90.99 & 67. & 32. 22 & 32.0 & Do. \\
\hline $\begin{array}{r}\text { Do.. } \\
\text { Florida } . . .\end{array}$ & 3395 & 2.334 & 94.29 & 62. & 37.53 & 29.6 & Do. \\
\hline $\begin{array}{c}\text { Florida } . . . \\
\text { Do }\end{array}$ & 3041 & 2. 880 & 99.69 & 67.13 & 32.87 & 31.5 & Brown. \\
\hline $\begin{array}{l}\text { Do } \\
\text { Do. }\end{array}$ & 3043 & 2. 018 & 98.70 & 68.61 & 31. 39 & 26.9 & Yellow. \\
\hline Do.. & 3044 & 2.966 & 93.95 & 71. & 28.31 & 31.0 & Brown \\
\hline Do.. & 3045 & 2. 531 & 98.26 & 69: 40 & 30.60 & 31.5 & Do. \\
\hline Do... & 3392 & 2.315 & 98. 33 & 67.85 & 32.15 & 24.2 & Yellow. \\
\hline Alabama.. & 3001 & 2.924 & 97.36 & & & 32.0 & Brown. \\
\hline Do.. & 3002 & 3. 068 & 97.23 & 68.34 & 31.66 & 24.7 & Do. \\
\hline Do.. & 3007 & 2.498 & 94.86 & 66.48 & 33.52 & 32.4 & Do. \\
\hline Do.. & 3008 & 3.127 & 94.10 & & 30.61 & 36.0 & Do. \\
\hline Do.... & 3363 & 3. 190 & 98.81 & 68.47 & 31.53 & 33. 3 & Do. \\
\hline Mississippi . & 3181 & 3. 034 & 96.50 & 69.50 & 30.50 & 34.5 & Do. \\
\hline Do.... & 3183 & 2.950 & 96.50 & 67. & 32.20 & 34.8 & Do. \\
\hline Do.. & 3184 & 2.792 & 91.20 & 73. 69 & 26.31 & 30.1 & Do. \\
\hline Do.. & 3185 & 2.113 & 70.00 & 74.60 & 25.40 & 38.2 & Do. \\
\hline Do.... & 3187 & 2. 868 & 97.80 & & 33.00 & 34.4 & Do. \\
\hline Louisiana.. & 3126 & 2. 993 & 99.29 & 69.34 & 30.66 & 33. 0 & Do. \\
\hline Do.. & 3127 & 2.775 & 90.03 & & 31.81 & 35.3 & Do. \\
\hline Do..... & 3441 & 3.104 & 95.65 & 72. 16 & 27.84 & 42.6 & Do. \\
\hline A rkansas.... & 3012 & & & & & 36.5 & Do. \\
\hline 1o.... & 3368 & 2.760 & 97.08 & 64.10 & 35. 90 & 33.8 & Do. \\
\hline Texas...... & 3310 & 3. 055 & 98.62 & 70.18 & 29.82 & 31.4 & Yellow. \\
\hline Do. & 3311 & 2.491 & 96.48 & 71.79 & 28.21 & 34.8 & Brown. \\
\hline Do & 3313 & 2.920 & 96.37 & & 26.49 & 34.8 & Do. \\
\hline Do. & 3314 & 2.841 & 98. 73 & 69. 78 , & 30. 22 , & 37.6 & Do. \\
\hline
\end{tabular}


Physical properties of oats-Continued.

\begin{tabular}{|c|c|c|c|c|c|c|c|}
\hline State. & $\begin{array}{l}\text { Serial } \\
\text { num. } \\
\text { ber. }\end{array}$ & $\begin{array}{l}\text { Weight } \\
\text { of } 100 \\
\text { grains. }\end{array}$ & $\begin{array}{l}\text { Clean } \\
\text { grain. }\end{array}$ & $\begin{array}{l}\text { Hulled } \\
\text { grain. }\end{array}$ & Hulls. & $\begin{array}{c}\text { Weight } \\
\text { per } \\
\text { bushel. }\end{array}$ & Color. \\
\hline & & Grams. & Per cent. & Per cent. & Per cent. & Pounds. & \\
\hline Texas ..... & 3315 & 3.030 & 93.86 & 70.74 & 29.26 & 41.6 & Brown. \\
\hline Do............... & 3316 & 2.903 & 92.90 & 71.22 & 28.78 & 37.3 & Do. \\
\hline Do................. & 3317 & 3.169 & 96.76 & 72. 78 & 27.22 & 39.7 & Do. \\
\hline Do.................. & 3430 & 2.981 & 96. 64 & 72.49 & 27.51 & 34.6 & Do. \\
\hline Colorado............... & 3020 & 2.958 & 86.61 & 69.76 & 30.24 & 48.8 & White. \\
\hline Do................. & 3021 & 2. 247 & ... ....... & 69.32 & 30.68 & 42.4 & Light. \\
\hline Do $\ldots . . . . . . . . . .$. & 3385 & 2.163 & 93.70 & 70.31 & 29.69 & 38.6 & White. \\
\hline Utah $\ldots \ldots \ldots \ldots \ldots$ & 3319 & 2.560 & 90.72 & 61.17 & 38.83 & 43.6 & White. \\
\hline Nevada ................ & 3205 & 2.019 & 95.05 & 66.01 & 33.99 & 41.1 & White. \\
\hline New Mexico........... & 3218 & 2.462 & 97.67 & 73. 21 & 26. 79 & 43.9 & Brown. \\
\hline 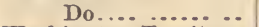 & 3420 & & & & & 39.6 & Mixed. \\
\hline Washington Territory. & 3341 & 3.255 & 98.48 & 72.91 & 27.09 & 43.5 & White. \\
\hline Do.................... & 3435 & 3. 148 & 97.16 & 79.28 & 20.72 & 43.2 & Light brown. \\
\hline Oregon $\ldots \ldots \ldots$ & 3275 & 2. 772 & 96.85 & 73. 09 & 26.91 & 46.9 & White. \\
\hline$\ldots \ldots \ldots$ & 3277 & 3. 786 & 97. 09 & 59.15 & 40.85 & 43.3 & Do. \\
\hline
\end{tabular}

From the preceling tables it appears that in the North white (includ. ing in this color yellow), black and white, and biack oats are principally sown, while in the South varieties of the red rust proof are almost entirely grown.

In the North the crop is put in in April or May and harvested in July or August. In the South it is sown from November to Jannary and harrested in May or June.

The difference in appearance is marked between the crops of these two sections of the country. The Southern oats are large, light, awned varieties, of reddish brown color, with inflated husks not nearly filled by the kernel. The Northern grain is smaller, more compact, not often awned, and with the husk in the better samples well filled out.

Notwithstanding these characteristics, we learn from averages of the results that the size and weight of the Southern clean kernel is rather larger than the Northern. Its fluffy husk, however, makes them lighter oats in weight per bushel.

The averages have been calculated for various sections as follows: The Northern States include all north of Maryland and Kentucky, together with Missouri, Montana, and Dakota; the Southern, all sonth of these; and the Pacific slope, Colorado, Nevada, New Mexico, Washington Territory, Oregon, and Utah; the Atlantic slope consists of the States east of the ()hio river and the Gulf; the.Western States, of those west of this line, excepting those on the Pacific slope. The same classification is preserved with the other cereals. 
Average physical properties of oats.

\begin{tabular}{|c|c|c|c|c|c|c|}
\hline State. & 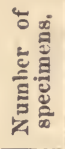 & 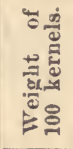 & 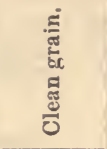 & 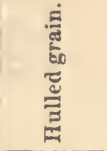 & $\underset{\underline{\Xi}}{\stackrel{i}{\Xi}}$ & 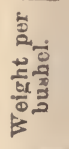 \\
\hline United States. & & 2.507 & $\begin{array}{l}\text { Per cent. } \\
\text { 95. } 00\end{array}$ & $\begin{array}{r}\text { Per cent. } \\
69,97\end{array}$ & $\begin{array}{c}\text { Per cent. } \\
30.03\end{array}$ & Lbs. \\
\hline Norther'n Siates. & 90 & 2.290 & 95.63 & 70.60 & 29.30 & $\begin{array}{l}37.2 \\
38.0\end{array}$ \\
\hline Southeru States .... & 66 & 2. 1128 & 94.38 & 69.08 & 30.42 & 34.5 \\
\hline Pacitic Slope ........ & 20 & 2. 737 & 94.93 & 69.52 & 30.48 & 43.2 \\
\hline Atlautio Slupe....... & 58 & 2.523 & 95.37 & 69. 69 & 30.31 & 37.0 \\
\hline Westeru States...... & 61. & 2.339 & 95.82 & 72.20 & 27. 80 & 37.8 \\
\hline Maine .............. & 3 & 2. 344 & 97.38 & 711.29 & $2 ! .71$ & 38.6 \\
\hline New Hampshire...... & 3 & 2.472 & 93.83 & 71.54 & 28.46 & 38.3 \\
\hline Vermont .............. & 4 & 2.474 & 94.97 & 69. 52 & 30.48 & 39.4 \\
\hline Connecsicut ........... & 4 & 2.110 & 95. 78 & 67.44 & 32.56 & 35.6 \\
\hline Rhorle Island........ & 1 & 3. $02 !$ & 97.16 & 67.27 & 3ذ. 73 & 32.3 \\
\hline New Tork........... & 8 & 2.571 & 95.32 & 71.0!) & 29.00 & 39.8 \\
\hline New Jerkey ......... & 2 & 2.371 & 98.50 & 72.75 & 27.25 & 36.6 \\
\hline Pennsylvauia............ & 4 & 2. 686 & 92. 24 & $68.5 j$ & 31.45 & 38.0 \\
\hline Ohio ................... & 9 & 2. 256 & 96.42 & 71.44 & 28.56 & 39.8 \\
\hline Michigan............. & 6 & 2. 767 & 96.22 & $7 \div 31$ & 27. 69 & 42.3 \\
\hline Indiana............. & 3 & 2. 388 & 93. 36 & 72.00 & $2 \times .00$ & 36.7 \\
\hline Illinois .............. & 9 & 2.3 .50 & 97.21 & 72.02 & 27.98 & 39.4 \\
\hline Wisconsill........... & 5 & 2. 487 & 94.81 & 70.77 & 29.23 & 39.0 \\
\hline Minuesota.......... & 9 & 2.416 & 96. 09 & 71.67 & 28.33 & 43. 6 \\
\hline Iowa .................. & 6 & 2. 245 & 96.99 & 71.63 & 28.37 & 38.4 \\
\hline Missouri.................. & 3 & 1.867 & 98.13 & 61.78 & 30.22 & 37.8 \\
\hline Nebranka.............. & 3 & 1. 763 & 97. $\times 7$ & 70.10 & 29.90 & $3: 1$ \\
\hline Dakota................ & 5 & 2.519 & 92.92 & 66.20 & 33. 80 & 43.3 \\
\hline Montana.............. & 3 & 2.410 & 91.06 & 70.54 & 29.46 & 30.0 \\
\hline Marylanil............ & 2 & 2.244 & 93.26 & 71.53 & 28.47 & 37.5 \\
\hline Delaware ............. & 1 & 1.976 & 98.04 & 69.69 & 30.41 & \\
\hline Virginia ............ & 3 & 2. $6: 7$ & 94.67 & 65.23 & 34. 77 & 39.2 \\
\hline West Virginia ....... & 4 & 2.500 & 95.94 & 6 ti. 48 & 33.52 & 39. 2 \\
\hline North Carolina ....... & 6 & 2. 496 & 94.52 & 70.37 & 29.63 & 39.8 \\
\hline South ('uruliua ....... & 8 & 2. 767 & 95.99 & 69.52 & $31 \% .43$ & 384 \\
\hline Kenturky ................ & 4 & 2. 249 & 94.88 & 69.49 & 30.01 & 31.7 \\
\hline Tenuessee......................... & 4 & 2. 089 & 95. 66 & 65.42 & 34.58 & 36.3 \\
\hline ia .................... & 7 & 2.619 & 93. 39 & 68.56 & 31. 44 & 31.6 \\
\hline 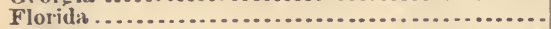 & 5 & $2.5+2$ & 97. 79 & 68.94 & 31.06 & 29.0 \\
\hline A labama $\ldots . . . . . . . . . . . . .$. & 5 & 2. $96 \mathrm{~L}$ & 96.47 & $6 \times .17$ & 31.83 & 31.7 \\
\hline 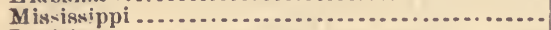 & 5 & 2. 751 & 90.40 & 70. 52 & 29.48 & 34.4 \\
\hline Louisiana & 3 & 2957 & 94.99 & 69.90 & 31). 10 & 36.9 \\
\hline 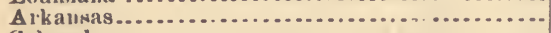 & 1 & 2. 760 & 97. 08 & 64. 10 & 35. 90 & 35.2 \\
\hline 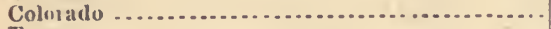 & $\delta$ & 2.442 & 90.16 & 69.80 & 30.21 & 43. 3 \\
\hline 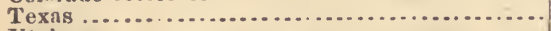 & 8 & $2.9 \pm 4$ & 96. 29 & 71.56 & 28.44 & 36.5 \\
\hline 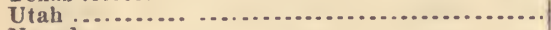 & 1 & 2.560 & 90. 72 & 61.17 & 38. 83 & 43.6 \\
\hline 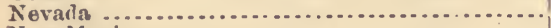 & 1 & 2.019 & 95. 05 & 66.01 & 33.99 & 411 \\
\hline 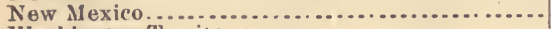 & 1 & 2.462 & 97.67 & 73. 21 & 26. 79 & 41.8 \\
\hline 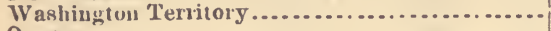 & 2 & 3.602 & 98.34 & 76.10 & 23.90 & 43.4 \\
\hline 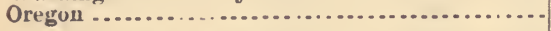 & 2 & 3.279 & 96.97 & 66.12 & 33. ₹8 & 45. 1 \\
\hline
\end{tabular}

Oats having the husk (pallets and at times glumes) allherent is necessarily lighter than wheat in weight per hundrel grains. The heaviest is from the Pacific slope, and the South ranks next, owing, as bas been said, to its large size. In weight per bushel, however, the fluffy husk of the Southern grain makes it the lowest in the country, while the Pacific slope retains the highest weight per bushel, as also size and weiglt per 100, showing it to have a plump, weli-filled grain.

The average for the country, 37.2 pounds, appears rather high in comparison with the most common legal weight, 32 pounds, but, as in the case of wheat, the determinations have been proved correct for the specimens examined, and are not mere estimates. The samples are, too, apparently fair averages, as the figures giviug percentages of clean grain in the specimens as received show that no particular pains was taken to prepare them for exhibition before reaching us. The range 
with this cereal is larger than with any other. The extremes in weight per 100 grains were, serial No. 3200, the lightest, from Nebraska, 1.582 grams, and serial No. 3277, from Oregon, the heariest, 3.786 grams. Cleanliness varied from 99.8 per cent. to 70.0 per cent., but of course had nothing to do with locality. The heaviest weight per bushel was found in specimens from Colorado, serial No. 3020, and Dakota, serial No. 3036, weighing 48.8 and 48.6 ponnds. The lightest were from Alabama, serial No. 3002, and from Florida, serial No. 30เ3, 24.7 and 26.9 pounds, respectively.

In milling oats the relation of kernel to waste is about one-half. Our results show that the relation of kernel to husk averages for the whole country 7 to 3, those from the Westeru States being a little less husky, and those from the South considerably more so. It is, however, the inflated nature of the husk in the Southern grain and the fact that the glumes or onter husk is often adherent that affects the weight per bushel more than the slightly larger proportion.

The extremes found were 79.28 per cent. of keruel in a specimen from Washington Territory, serial No. 3435, and $5 \bar{J} .37$ per cent. in one from Dakota, serial No.3391. Washington and Uregon sustain their reputation for fine looking grain, while the small proportion of kernel in the Dakota specimen is due entirely to cutting before it was quite ripened. It is hardly a fair example, other specimens from the Territory reaching orer $\mathbf{7 0 . 0}$ per cent. of kernel.

In weight per bushel the warm climate of the South so affects the form of the grain as to lower its average 2.7 pounds. This is hardly as large as would be expected, and leads to the conclusion that the climate has a greater effect than some other characteristics. One of these is yield, which, from an arerage of 30 bushels in the North, falls to about 10 in the South, aud, as has been said, the color and shape of the grain is much changed.

CHEMICAL COMPOSITION.

In examining the physical relations of the specimens they were separated into kernel and husk, and for several reasons they were separately submitted to analysis with, as it appears, results furnishing much more information than would otherwise have been obtained. Corn, wheat, and rye were analyzed in the clean kernel, and comparison is more readily made between them and oats. The variations, independent of the proportion of husk, are easily arrived at, and since the analysis of the hull and its relative amount are given it is easy to calculate for any specimen its composition as it would be used for feed.

The data obtained are here presented, together with averages derived therefrom : 
Composition of American oats, arranged by States.

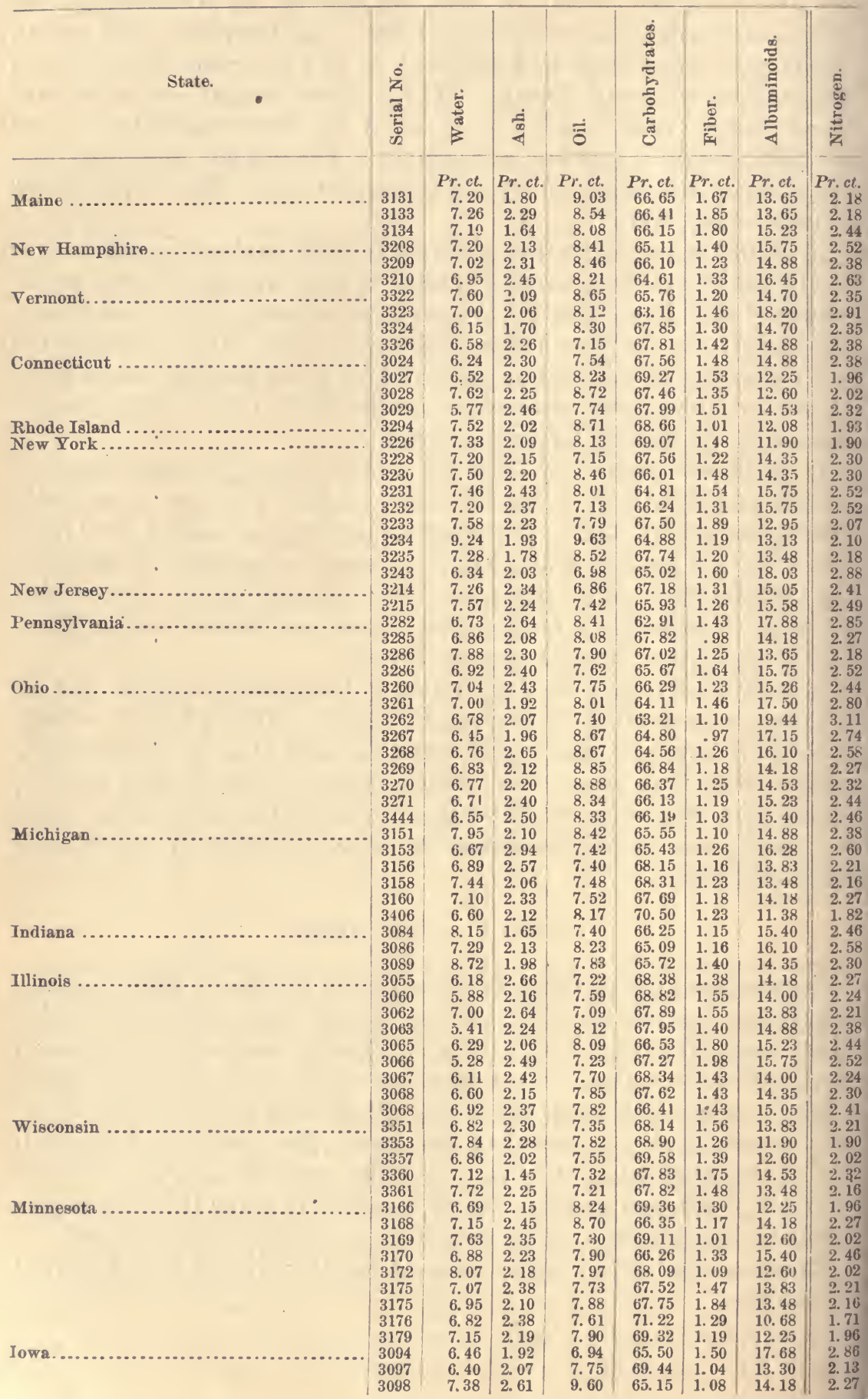


Composition of American oats, asranged by States-Continued.

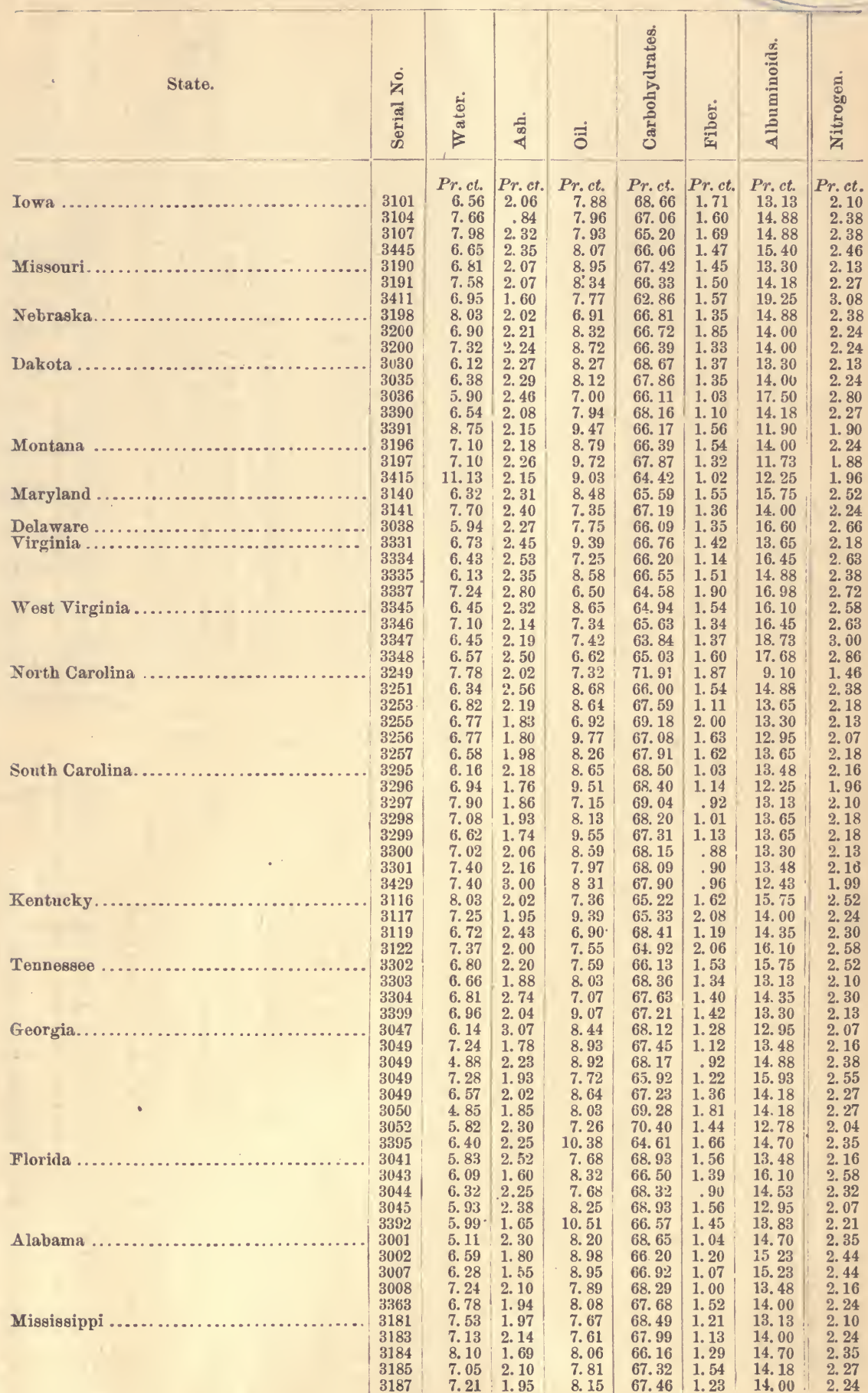


Composition of American oats, arranged by States-Continued.

\begin{tabular}{|c|c|c|c|c|c|c|c|c|}
\hline State. & 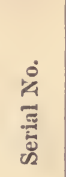 & 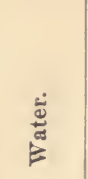 & $\frac{\pi}{4}$ & $\overline{0}$ & 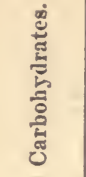 & 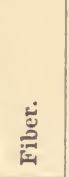 & 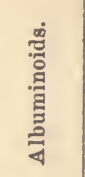 & 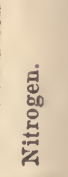 \\
\hline $\begin{array}{l}\text { Louisiana........ } \\
\text { Arkansas ......... } \\
\text { Texas............ } \\
\\
\text { Colorado......... } \\
\text { Utah ............ } \\
\text { Nerada........... } \\
\text { New Mexico..... } \\
\text { Washington ...... } \\
\text { Oregon ............ } \\
\text { California ......... }\end{array}$ & $\begin{array}{l}3126 \\
3127 \\
3441 \\
31112 \\
3368 \\
3310 \\
3.111 \\
3313 \\
3314 \\
3315 \\
33116 \\
3317 \\
3130 \\
3020 \\
3021 \\
33 \times 5 \\
3583 \\
3319 \\
3321 \\
3205 \\
3218 \\
34: 20 \\
3341 \\
3435 \\
3275 \\
3277 \\
3016 \\
3374 \\
3378 \\
3380 \\
3382\end{array}$ & $\begin{array}{r}\text { Per ct. } \\
9.50 \\
8.00 \\
6.85 \\
4.67 \\
6.94 \\
7.08 \\
6.92 \\
8.57 \\
6.70 \\
7.14 \\
6.80 \\
6.45 \\
7.10 \\
4.80 \\
5.08 \\
6.56 \\
7.20 \\
6.05 \\
7.30 \\
6.80 \\
6.61 \\
7.05 \\
7.08 \\
6.55 \\
.6 .72 \\
7.01 \\
7.95 \\
7.02 \\
6.58 \\
6.52 \\
7.12\end{array}$ & $\begin{array}{l}P . c t . \\
2.20 \\
2.10 \\
2.10 \\
2.10 \\
2.14 \\
1.74 \\
2.08 \\
2.15 \\
1.86 \\
2.26 \\
1.82 \\
2.10 \\
2.30 \\
2.08 \\
2.40 \\
2.25 \\
2.45 \\
2.37 \\
2.40 \\
2.27 \\
2.12 \\
2.50 \\
1.79 \\
1.55 \\
2.28 \\
2.42 \\
1.93 \\
1.58 \\
1.79 \\
2.14 \\
1.35\end{array}$ & $\begin{array}{r}\text { Per ct. } \\
8.18 \\
7.83 \\
8.25 \\
8.12 \\
7.71 \\
8.09 \\
11.20 \\
9.06 \\
8.80 \\
8.75 \\
8.08 \\
8.19 \\
7.45 \\
7.27 \\
8.67 \\
7.67 \\
7.59 \\
8.17 \\
8.81 \\
9.72 \\
989 \\
9.43 \\
7.99 \\
10.57 \\
8.89 \\
7.87 \\
8.83 \\
9.67 \\
10.10 \\
9.11 \\
9.32\end{array}$ & $\begin{array}{c}\text { Per ct. } \\
64.99 \\
67.72 \\
66.93 \\
69.35 \\
65.83 \\
68.07 \\
65.34 \\
6 . .82 \\
67.26 \\
67.58 \\
66.03 \\
68.63 \\
67.81 \\
66.82 \\
68.48 \\
65.75 \\
6 \times .40 \\
69.71 \\
66.89 \\
66.21 \\
66.02 \\
66.30 \\
71.56 \\
68.36 \\
68.73 \\
60.80 \\
66.33 \\
67.91 \\
70.02 \\
66.35 \\
68.86\end{array}$ & $\begin{array}{l}P . c t . \\
1.13 \\
1.05 \\
1.34 \\
1.93 \\
1.63 \\
1.12 \\
1.55 \\
1.65 \\
1.03 \\
1.14 \\
1.20 \\
.83 \\
1.16 \\
1.00 \\
1.14 \\
1.10 \\
1.17 \\
1.62 \\
1.82 \\
1.17 \\
1.88 \\
1.59 \\
1.95 \\
1.07 \\
1.48 \\
2.07 \\
1.83 \\
1.86 \\
1.88 \\
1.70 \\
1.27\end{array}$ & $\begin{array}{r}\text { Per ct. } \\
14.00 \\
13.30 \\
14.53 \\
13.63 \\
15.75 \\
13.30 \\
12.95 \\
15.75 \\
14.35 \\
13.13 \\
13.48 \\
13.30 \\
14.16 \\
18.03 \\
13.13 \\
16.63 \\
13.13 \\
12.08 \\
12.78 \\
13.83 \\
13.48 \\
13.13 \\
9.63 \\
11.90 \\
11.90 \\
13.83 \\
13.13 \\
11.73 \\
9.63 \\
14.18 \\
12.08\end{array}$ & $\begin{array}{l}P . c t . \\
2.24 \\
2.13 \\
2.32 \\
2.21 \\
2.52 \\
2.13 \\
2.07 \\
2.52 \\
2.30 \\
210 \\
2.16 \\
2.13 \\
2.27 \\
2.88 \\
2.10 \\
2.66 \\
2.10 \\
1.93 \\
2.14 \\
2.21 \\
2.16 \\
2.10 \\
1.54 \\
1.90 \\
1.90 \\
2.21 \\
2.10 \\
1.88 \\
1.54 \\
2.27 \\
1.93\end{array}$ \\
\hline
\end{tabular}

Composition of hulls of American oals, arranged by States.

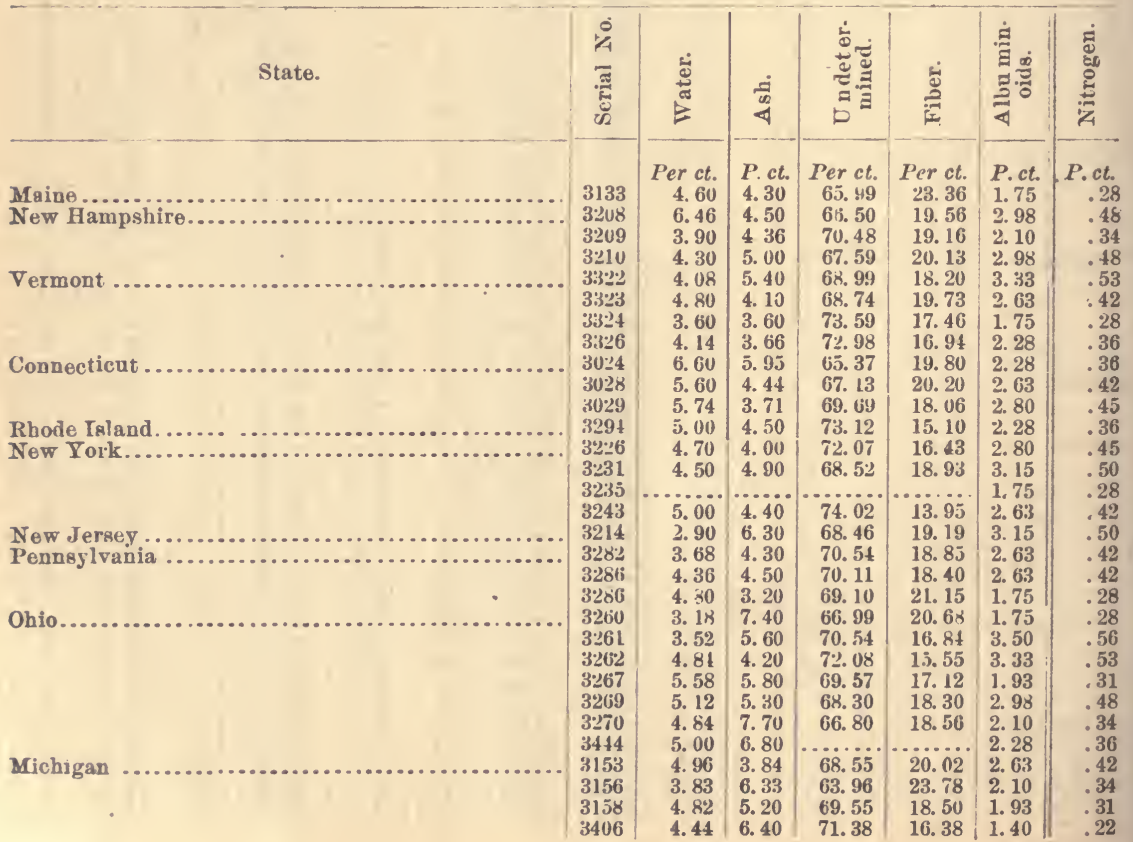


Composition of hulls of American oats, arranged by States-Continued.

\begin{tabular}{|c|c|c|c|c|c|c|c|}
\hline States. & 苟 & 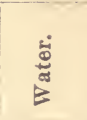 & $\frac{\text { के }}{4}$ & 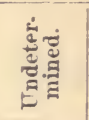 & 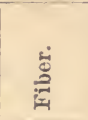 & 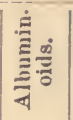 & 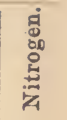 \\
\hline $\begin{array}{l}\text { Indiana . } \\
\text { llliwois.. }\end{array}$ & 3084 & $\begin{array}{r}\text { Per ct. } \\
4.80\end{array}$ & $\begin{array}{l}\text { P. ct. } \\
\text { 5. } 20\end{array}$ & $\begin{array}{r}\text { Per ct. } \\
\quad 68.17\end{array}$ & $\begin{array}{l}\text { Per et. } \\
19.38\end{array}$ & $\begin{array}{l}P . c t . \\
2.45\end{array}$ & $\begin{array}{r}\text { P. ct. } \\
.39\end{array}$ \\
\hline ....... & $\begin{array}{l}3055 \\
3060\end{array}$ & 4.42 & 7,10 & 6480 & 21.40 & 2.10 & \\
\hline 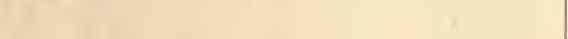 & 3062 & 7. 70 & 5. 70 & & 14.56 & 4. 73 & $\begin{array}{l}.36 \\
.76\end{array}$ \\
\hline & 3063 & 15. 16 & 7.80 & & 21.44 & 2.98 & \\
\hline & 3167 & 5. 16 & 7.20 & & & 2. 28 & \\
\hline & $\begin{array}{l}31168 \\
31168\end{array}$ & $\begin{array}{l}\text { 4. } 56 \\
5.12\end{array}$ & $\begin{array}{l}7.44 \\
5.48\end{array}$ & 1 & 14. 14 & 2.45 & .39 \\
\hline Wisconsin & 3353 & 4.40 & $\begin{array}{l}5.88 \\
7.10\end{array}$ & $\begin{array}{l}6.5 .78 \\
70.40\end{array}$ & $\begin{array}{l}21.12 \\
15.45\end{array}$ & $\begin{array}{l}2.10 \\
2.63\end{array}$ & .34 \\
\hline & 3357 & 4. 70 & 6. 80 & & 17.71 & 1.58 & 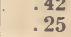 \\
\hline & $\begin{array}{l}3: 360 \\
33 t i 1\end{array}$ & 3.56 & 5.80 & 71.00 & 17.54 & 2.10 & .34 \\
\hline Minnesota & $\begin{array}{l}33 \hbar 1 \\
3 i 66\end{array}$ & $\begin{array}{l}2.20 \\
4.71\end{array}$ & $\begin{array}{l}6.50 \\
7.10\end{array}$ & $\begin{array}{l}70.35 \\
64.41\end{array}$ & $\begin{array}{l}19.20 \\
21 . \times 5\end{array}$ & $\begin{array}{l}1.75 \\
1.93\end{array}$ & .28 \\
\hline & 3170 & 4.35 & 6.21 & 68.27 & 18.44 & 2.63 & $\begin{array}{l}.31 \\
.42\end{array}$ \\
\hline & $\begin{array}{l}3172 \\
3175\end{array}$ & 5. 36 & 5.40 & 68.75 & 18.04 & 2.45 & .39 \\
\hline Iowa. & $\begin{array}{l}3175 \\
3176\end{array}$ & $\begin{array}{l}\text { 4. } 96 \\
6.54\end{array}$ & $\begin{array}{l}7.35 \\
7.00\end{array}$ & 67.70 & 16. 76 & $\begin{array}{l}1.75 \\
2.10\end{array}$ & $\begin{array}{l}.28 \\
.34\end{array}$ \\
\hline Iow & 3098 & 5.20 & $8.4 \mathrm{U}$ & & ..... & 1.75 & .28 \\
\hline & $\begin{array}{l}3104 \\
3107\end{array}$ & 4.50 & 6.22 & 0 & 22.14 & $2.4 j$ & .39 \\
\hline Missour & $\begin{array}{l}3107 \\
3191\end{array}$ & $\begin{array}{l}6.00 \\
5.13\end{array}$ & $\begin{array}{l}6.66 \\
6.55\end{array}$ & $\begin{array}{l}69.37 \\
69.11\end{array}$ & 16.22 & $\begin{array}{l}1.75 \\
2.63\end{array}$ & .28 \\
\hline Nebraska... & 3411 & 4. 30 & 5.50 & 70.60 & 15.87 & $\begin{array}{l}2.03 \\
3.33\end{array}$ & .42 \\
\hline $\begin{array}{l}\text { Nebraska } \\
\text { Dakota... }\end{array}$ & $\begin{array}{l}3198 \\
3035\end{array}$ & 4. 10 & 7.80 & 68.40 & 17 & 2.10 & .34 \\
\hline $20 \mathrm{ang}$ & $\begin{array}{l}3035 \\
3390\end{array}$ & 5,40 & 7.70 & 66.50 & $\begin{array}{l}18.12 \\
18.12\end{array}$ & $\begin{array}{l}1.75 \\
2.28\end{array}$ & .28 \\
\hline $\mathbf{M}$ & 3197 & 3.00 & 9.50 & 65.82 & 17.65 & 4.03 & .64 \\
\hline Maryland. & 3415 & 4.40 & 4.48 & 5 & 19. & 1. 75 & .28 \\
\hline & \begin{tabular}{|l|}
3140 \\
3141
\end{tabular} & 4.40 & 5.62 & 67. & 17. 43 & 4. 73 & .76 \\
\hline Dela & $\begin{array}{l}3141 \\
30: 38\end{array}$ & 4.00 & 4.62 & 64.68 & $\begin{array}{l}24.42 \\
16.54\end{array}$ & $\begin{array}{l}2.28 \\
3.15\end{array}$ & $\begin{array}{l}.36 \\
.50\end{array}$ \\
\hline Virg & 3334 & 3.08 & 3.90 & 74.41 & 16. 16 & 2.45 & .39 \\
\hline & 3335 & 4. 06 & 5.50 & 69. & 17. & 2.98 & .48 \\
\hline West Virginia. & $\begin{array}{l}3337 \\
3345\end{array}$ & $\begin{array}{l}5.10 \\
4.30\end{array}$ & $\begin{array}{l}3.70 \\
5.60\end{array}$ & 64. & $\begin{array}{l}23.42 \\
20.62\end{array}$ & 2.81 & .45 \\
\hline & 3347 & $\begin{array}{l}4.76 \\
3.76\end{array}$ & $\begin{array}{l}0.00 \\
3.60\end{array}$ & 72.32 & $\begin{array}{l}20.62 \\
17.69\end{array}$ & $\begin{array}{l}2.10 \\
2.63\end{array}$ & \\
\hline North Carolina... & $\begin{array}{l}3249 \\
3 * 51\end{array}$ & 5.80 & 4. 70 & 70.61 & 35.04 & 3. 85 & 62 \\
\hline & 3253 & 4.30 & 7.50 & 69.86 & 15.89 & $\begin{array}{l}2.80 \\
2.45\end{array}$ & .45 \\
\hline & $3: 55$ & 5.40 & 6 & 72 & & $\begin{array}{l}2.45 \\
3.15\end{array}$ & $\begin{array}{r}.39 \\
59\end{array}$ \\
\hline (1) & 3256 & 4.76 & 5.00 & 68. & 19. & 2.28 & $\begin{array}{l}.50 \\
.36\end{array}$ \\
\hline Sunth Carolina & 3257 & 482 & 5.90 & 71. & 15.37 & 2. 28 & .36 \\
\hline Sunth Carolina & $3: 95$ & $3.86^{\circ}$ & 3. & 76. & 13.50 & 2.10 & .34 \\
\hline & 3297 & 2.98 & 5. & & 14. & 1.75 & .28 \\
\hline & $\begin{array}{l}3299 \\
30110\end{array}$ & +10 & 5.40 & 73. & 13 & 2.98 & .48 \\
\hline & $\begin{array}{l}3010 \\
3402\end{array}$ & $\begin{array}{l}4.20 \\
6.00\end{array}$ & $\begin{array}{l}8.10 \\
5.80\end{array}$ & $\begin{array}{l}70 . \\
71 .\end{array}$ & $\begin{array}{l}15.40 \\
11.91\end{array}$ & $\begin{array}{l}1.75 \\
5.08\end{array}$ & .28 \\
\hline Kentucky. & 3116 & 5.10 & 4. & 64. & 23.55 & 1.75 & .28 \\
\hline & 3117 & 17.40 & 4. & 57. & 17.17 & 2. 80 & .45 \\
\hline ressee... & 3122 & 9.90 & & 60. & 19.22 & 4. & 78 \\
\hline esseo.. & 3304 & 8.90 & 6. & 67. & 14. & 2.45 & 39 \\
\hline Georgia. & $\begin{array}{l}3309 \\
3047\end{array}$ & $\begin{array}{l}4.86 \\
5.71\end{array}$ & $\begin{array}{l}5.50 \\
5.31\end{array}$ & $\begin{array}{l}65 . \\
68 .\end{array}$ & 3 & $\begin{array}{l}2.10 \\
2.45\end{array}$ & $\begin{array}{r}34 \\
39\end{array}$ \\
\hline & 3049 & 5.96 & 5.7 & $\begin{array}{l}68 . \\
70 .\end{array}$ & $\begin{array}{l}18 . \\
16 .\end{array}$ & $\begin{array}{l}2.45 \\
1.75\end{array}$ & $\begin{array}{l}.39 \\
.28\end{array}$ \\
\hline - & 304 & 5. & 5. & 71. & 15 & 2. & 39 \\
\hline & 305 & 5. & & 6 & & 2. & 34 \\
\hline & 3052 & 5.80 & 4. & 6 & & 2. & 39 \\
\hline Florida.... & $\begin{array}{l}3395 \\
3044\end{array}$ & 502 & 6. & 70 & 6 & 4. & 67 \\
\hline & $\begin{array}{l}3044 \\
3045\end{array}$ & 5.50 & 1.9 & 73. & 16. 58 & 2. 63 & 42 \\
\hline & $\begin{array}{l}30+5 \\
3392\end{array}$ & $\begin{array}{l}5.78 \\
3.30\end{array}$ & $\begin{array}{l}2.9 \\
3.8\end{array}$ & $\begin{array}{l}74 \\
75\end{array}$ & $\begin{array}{l}0 \\
6\end{array}$ & $\begin{array}{l}1.75 \\
2.45\end{array}$ & $\begin{array}{l}.28 \\
.39\end{array}$ \\
\hline Alabama. & 3002 & 14. 94 & 48 & 63. & 14 & 2.28 & 36 \\
\hline ippi... & $3: 363$ & 4.50 & 5.1 & 68. & & 2. & 34 \\
\hline sippi . & $31\}$ & 5. & & 6 & & 2. & 42 \\
\hline isiana... & 3187 & 5. & & & & 1. & .31 \\
\hline & 3126 & 15. & 4. & 58. & 19 & 2. & 34 \\
\hline 8... & & & & & & & .36 \\
\hline $18 \ldots$. & $\begin{array}{l}3012 \\
3310\end{array}$ & $\begin{array}{l}6.00 \\
4.40\end{array}$ & 5. & $6 \div$ & 16 & 2. & .48 \\
\hline & 3311 & $\begin{array}{l}4.40 \\
4.20\end{array}$ & 6. & $\begin{array}{l}66 . \\
72 .\end{array}$ & $\begin{array}{l}20 \\
14\end{array}$ & $\begin{array}{l}1.05 \\
2.63\end{array}$ & .42 \\
\hline & 3313 & 4.00 & 7. & 72.10 & 14. 70 & 2.10 & .34 \\
\hline & 33 & 3. & 7. & & & & \\
\hline 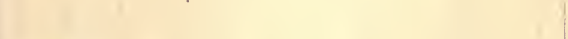 & & & & & & & \\
\hline Co & & & & & & 1. & 0 \\
\hline Colorado... & 3385 & 4. & 7. & & & 2. & .42 \\
\hline & $\begin{array}{l}3583 \\
3321\end{array}$ & $\begin{array}{l}4.04 \\
4.70\end{array}$ & 5.40 & 65.92 & $\begin{array}{l}22.54 \\
20.59\end{array}$ & $\begin{array}{l}2.10 \\
3.50\end{array}$ & $\begin{array}{l}.34 \\
.56\end{array}$ \\
\hline ngton & $3+35$ & 5.20 & 7.1 & 67. & & 1.93 & 31 \\
\hline 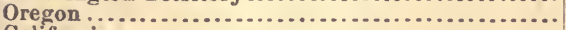 & 3277 & 5. 12 & & & & 1.75 & 0 \\
\hline fornia. & 3374 & 4.20 & 6.02 & 71.63 & 16.40 & 1.75 & .28 \\
\hline
\end{tabular}


Average composition of American oats, arranged by States.

\begin{tabular}{|c|c|c|c|c|c|c|c|c|}
\hline States. & 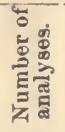 & $\frac{\vec{\Phi}}{\text { है }}$ & $\stackrel{\dot{m}}{4}$ & छే் & 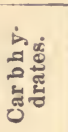 & $\frac{\dot{\Phi}}{\stackrel{\Xi}{|c|}}$ & 爮 & 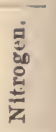 \\
\hline United States... & 179 & 6.93 & 2.15 & 814 & 67.09 & 1. 38 & 14.31 & 2.28 \\
\hline Atlantic Slope. & 64 & 6.84 & 2. 17 & 8.22 & 67.10 & 1.37 & 14. 30 & 2.28 \\
\hline 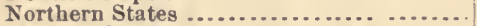 & 92 & 7. 07 & 2.19 & 8.02 & 66.88 & 1.37 & 14.47 & 2. 32 \\
\hline Western States ... & 54 & 6.98 & 2. 19 & 7.91 & 67.06 & 1.37 & 14.49 & 2. 32 \\
\hline 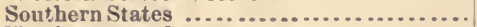 & 69 & 6. 79 & 2. 12 & 8.23 & 67. 22 & 1.35 & 14.29 & 2.28 \\
\hline Northwestern States .................... & 8 & 7.38 & 2.23 & 8.54 & 66. 96 & 1.28 & 1361 & 2. 18 \\
\hline 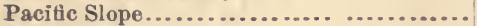 & 18 & 6. 71 & 2.10 & 8.87 & 67. 78 & 1.53 & 13. 01 & 2. 08 \\
\hline 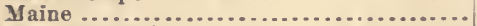 & 3 & 7.19 & 1.91 & 8.55 & 66.40 & 1.77 & 14.18 & 2. 27 \\
\hline 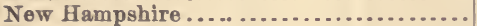 & $\mathbf{3}$ & 7.06 & 2.30 & 8. 36 & 65. 27 & 1.32 & 15. 69 & 2.51 \\
\hline 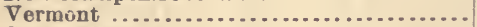 & 4 & 6. 33 & 2.00 & 8. 06 & 66. 15 & $1: 34$ & 15.62 & 2. 50 \\
\hline Connecticut ......... & 4 & 6. 54 & 2. 30 & 8. & 68.07 & 1.47 & 13.56 & 2.17 \\
\hline Rhode Island... & 1 & 7.52 & 2. 02 & 8.71 & 68 & 1.01 & 12.08 & 1.93 \\
\hline New York..... & 9 & 7.46 & 2.14 & 8. & 66. & & 14.41 & 2. 31 \\
\hline Jersay. & 2 & 7.42 & 2.29 & 7. & 6 & 9 & 15. 31 & 2.45 \\
\hline sylvania... & 4 & 7.10 & 2. 36 & 8. & 65 & 1.32 & 15. 37 & 2.46 \\
\hline ....... & 9 & 6.76 & 2.25 & 8. & 6 & 1.19 & 16.09 & 2.57 \\
\hline Mi & 6 & 7.11 & 2. 35 & 7. & 67 & 1.19 & 14. & 2. 24 \\
\hline Ind & 3 & 8.05 & 1. 92 & $\ddot{7}$ & & 1. 24 & 15.28 & 2.45 \\
\hline IIl & 9 & 6.19 & 2. 34 & 7. & 6 & 1.55 & 14.59 & 2. 34 \\
\hline$W i$ & 5 & 7. 27 & 2.06 & 7. & 68. & 1.49 & 13. & 2. 12 \\
\hline ................. & 9 & 7.16 & 2. & 7. & 6 & 1.30 & 13.03 & 2.09 \\
\hline . . . . & 7 & 7.01 & 2.03 & 8. & 2 & 1.44 & 14 & 2. 37 \\
\hline .......... & 3 & 7. 11 & 1 & 8. & 6 & 1.51 & 15 & 2.49 \\
\hline Nebraska ..... & 3 & 7.42 & 2.16 & 7. & 66 & 1.51 & 14. 29 & 2.29 \\
\hline . & 5 & 6. 74 & & 8. & & j. 28 & 14.28 & 2. 27 \\
\hline & 3 & 8.44 & 2. & 9. & 66 & 1. 29 & 12.66 & 2. 03 \\
\hline 等, & 2 & 7.01 & & & & 1.46 & 14.87 & \\
\hline $\mathrm{D}$ & 1 & 5.94 & 2. & 7. & 66 & 1.35 & 16.60 & \\
\hline $\overrightarrow{v i}$ & 4 & 6.63 & 2.43 & 7. & & 1.49 & 15.49 & 2.48 \\
\hline ia.... & 4 & 6. 64 & 2. & 7. & 6 & 1.46 & 17.24 & 2. 76 \\
\hline No & 6 & 6.84 & & 8. & & 1.63 & 12.92 & 2.07 \\
\hline n? & 8 & 7.07 & 2. & 8. & 6 & 1. & 13 & 2. 11 \\
\hline Ke & 4 & 7.34 & 2.10 & 7. & & 1.74 & 15.05 & 2.41 \\
\hline - & 4 & 6. 81 & 2. & 8. & 67. & L. 42 & 14 & 2.26 \\
\hline Ge & 8 & 6.15 & 2. 28 & 8.54 & 67.65 & 1.35 & 14.13 & 2.26 \\
\hline & 5 & 6. & 2. 08 & 8. & 67. & 1.37 & 14.18 & 2.27 \\
\hline ma................. & 5 & 6.40 & 1.94 & 8. & 67.55 & 1.16 & 14.53 & 2. 33 \\
\hline Mississippi ........ & 5 & 7.41 & 1. & 7. & 67.48 & 1.28 & 14. & \\
\hline ............. & 3 & 8.12 & 2.13 & 8. & 66.55 & 1.17 & 13.94 & \\
\hline Arkar & 2 & 5. 80 & 1.12 & 7. & 67.59 & 1.78 & 14. 79 & 2. 37 \\
\hline$\ldots$ & 8 & 7.16 & 2.04 & 8. & 67.08 & 1.21 & 13.80 & 2. 21 \\
\hline $\mathbf{C}$ & 4 & 6. 06 & 2.31 & 7. & 67.50 & 1.10 & 15.23 & 2.44 \\
\hline Uta & 2 & 6. 67 & 2. 39 & 8.49 & 68. 30 & 1.72 & 12.43 & 1.99 \\
\hline & $\overline{1}$ & 6. 80 & 2.27 & 9. & 66.21 & 1.17 & 13. 83 & 2.21 \\
\hline exico .......... & 2 & 6.83 & 2. 31 & 9. & 66.16 & 1.73 & 13. 31 & 2.14 \\
\hline ington Territor & 2 & 6. 82 & 1. & 9. & 69.96 & 1.51 & 10.76 & 1.72 \\
\hline & 2 & 6.86 & 2. 35 & 8.38 & 67.77 & 1.78 & 12.86 & 2.06 \\
\hline California & $\mathbf{5}$ & 7. 08 & 1. 76 & 9.40 & 67.90 & 1. 71 & 12.15 & 1.94 \\
\hline
\end{tabular}

Average composition of hulls of oats, arranged by States.

\begin{tabular}{|c|c|c|c|c|c|c|c|c|}
\hline State. & - & 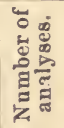 & 岕 & $\ddot{x}^{\infty}$ & 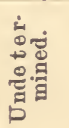 & 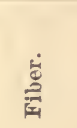 & 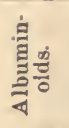 & 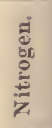 \\
\hline \multirow{2}{*}{\multicolumn{2}{|c|}{ 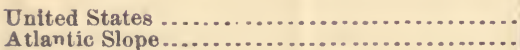 }} & 100 & 5. 22 & 5. 59 & 68.83 & 17. 88 & 2. 48 & .40 \\
\hline & & 43 & 4. & & 70.35 & 17. & 2. 64 & 42 \\
\hline \multicolumn{2}{|c|}{$\begin{array}{l}\text { Atlantic Slope } \\
\text { Northern States..... }\end{array}$} & 52 & 4. & 5. & 68. & 18. & 2.48 & 10 \\
\hline \multirow{2}{*}{\multicolumn{2}{|c|}{ 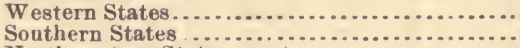 }} & 33 & 4. & & 67. & 18. & 2. & 38 \\
\hline & & 43 & 5.7 & 5. & & 17. & & \\
\hline \multirow{2}{*}{\multicolumn{2}{|c|}{ 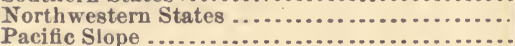 }} & 17 & 4. & & 68 & 18. & 2. & \\
\hline & & 2 & 5. 1 & 5. & 67. & 1915 & & \\
\hline \multicolumn{2}{|c|}{ Maine } & 1 & 4. & & 65. & 23. & 1. & \\
\hline \multicolumn{2}{|c|}{ 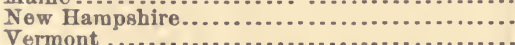 } & 3 & & 4. & 68. & 19. 62 & & \\
\hline \multirow{2}{*}{\multicolumn{2}{|c|}{$\begin{array}{l}\text { Vermont } \ldots \ldots \ldots \\
\text { Connecticut }\end{array}$}} & 4 & & & 71. & 18. & & 40 \\
\hline & & 3 & 5. & 4. & 67. & 19. 35 & & \\
\hline \multirow{2}{*}{\multicolumn{2}{|c|}{ 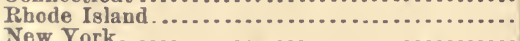 }} & 1 & & & & 15.10 & & \\
\hline & & 3 & 4. & 4. & 71. & 16.44 & & \\
\hline \multicolumn{2}{|c|}{ 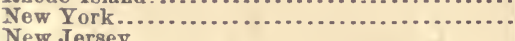 } & 1 & 2. & & & 19.19 & & \\
\hline \multicolumn{2}{|c|}{ 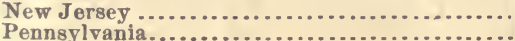 } & 3 & 4. 28 & 4. 00 & 69.92 & 19.47 & 2.33 & \\
\hline
\end{tabular}


- Average composition of hulls of oats, arranged by States-Continued.

\begin{tabular}{|c|c|c|c|c|c|c|c|}
\hline State. & 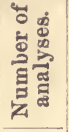 & 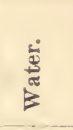 & बं & 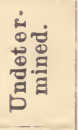 & 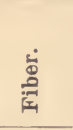 & 离苟 & 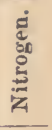 \\
\hline 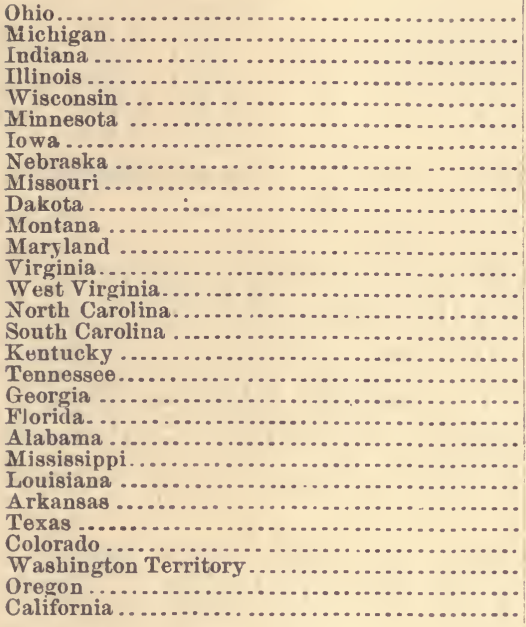 & 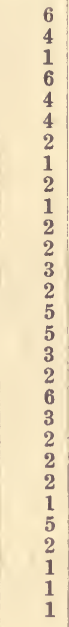 & $\begin{array}{r}4.51 \\
4.51 \\
4.80 \\
7.01 \\
3.71 \\
5.24 \\
5.25 \\
4.10 \\
4.72 \\
5.40 \\
7.40 \\
4.20 \\
4.08 \\
4.03 \\
5.02 \\
4.23 \\
10.80 \\
6.88 \\
5.53 \\
4.86 \\
9.72 \\
5.30 \\
10.30 \\
6.00 \\
4.06 \\
4.17 \\
5.20 \\
5.12 \\
4.20\end{array}$ & $\begin{array}{l}6.00 \\
5.44 \\
5.20 \\
6.85 \\
6.58 \\
6.43 \\
6.43 \\
7.80 \\
6.23 \\
7.70 \\
6.99 \\
5.12 \\
4.87 \\
4.60 \\
5.92 \\
5.64 \\
5.00 \\
6.00 \\
5.13 \\
2.19 \\
4.98 \\
7.28 \\
4.90 \\
5.14 \\
7.22 \\
6.62 \\
7.16 \\
4.80 \\
6.02\end{array}$ & $\begin{array}{l}69.05 \\
68.36 \\
68.17 \\
64.95 \\
70.22 \\
67.28 \\
67.04 \\
68.40 \\
69.85 \\
66.50 \\
68.04 \\
66.24 \\
69.77 \\
69.85 \\
70.48 \\
73.47 \\
60.87 \\
66.61 \\
69.51 \\
74.44 \\
65.68 \\
67.95 \\
63.38 \\
69.08 \\
71.01 \\
66.82 \\
67.37 \\
68.37 \\
71.63\end{array}$ & $\begin{array}{l}17.84 \\
19.67 \\
19.38 \\
18.43 \\
17.48 \\
18.77 \\
19.18 \\
17.60 \\
16.22 \\
18.12 \\
18.38 \\
20.94 \\
19.03 \\
19.16 \\
15.78 \\
13.93 \\
20.18 \\
18.24 \\
17.28 \\
15.51 \\
17.43 \\
17.19 \\
19.23 \\
16.80 \\
15.96 \\
20.03 \\
18.34 \\
19.96 \\
16.40\end{array}$ & $\begin{array}{l}2.60 \\
2.02 \\
2.45 \\
2.81 \\
2.01 \\
2.28 \\
2.10 \\
2.10 \\
2.98 \\
2.28 \\
2.89 \\
3.50 \\
2.75 \\
2.36 \\
2.80 \\
2.73 \\
3.15 \\
2.27 \\
2.57 \\
2.28 \\
2.19 \\
2.28 \\
2.19 \\
2.98 \\
1.75 \\
2.36 \\
1.93 \\
1.75 \\
1.75\end{array}$ & $\begin{array}{l}.42 \\
.32 \\
.39 \\
.45 \\
.32 \\
.36 \\
.34 \\
.34 \\
.48 \\
.36 \\
.46 \\
.56 \\
.44 \\
.38 \\
.45 \\
.44 \\
.50 \\
.36 \\
.41 \\
.36 \\
.35 \\
.36 \\
.35 \\
.48 \\
.28 \\
.38 \\
.31 \\
.28 \\
.28\end{array}$ \\
\hline
\end{tabular}

The chemical composition of the specimens appears from the preceding data to be rather surprising. It was reasouable to suppose that as oats deteriorate so readily, and are apparantly so easily influenced by their environment, great variations would be found in their composition under different climatic conditions, as is the case with wheats. Brewer remarks in his census report that a hundred or more analyses would be requisite to set at rest all questions in regard to this grain, and that they would be an extremely valuable contribution to our knowledge of the comparative nutritive values of the oats grown in different portious of the United States and their relative economic values. One hundred and seventy-nine analyses have been made, and we learn that there is not that variation in the oat kernel itself which was expected to be due to climatic condition. The proportion of husk to kernel and the compactness of the grain prove to be the all-important factors, and the weight per bushel the best means of judging of the value of the grain.

The only peculiarities noticed are that the eighteen specimens from the Pacific slope are lower in albuminoids and richer in fiber than the averages for other parts of the country. The arerage for the hulls from the West show the presence of more ash than in those from the East, and more fiber, and, like the kernels, they are slightly deficient in al- 
buminoids. Actual analysis of the mixed remainder from the individual analyses of the hulls furnished the following results:

\begin{tabular}{|c|c|c|c|}
\hline t & North. & South. & West. \\
\hline 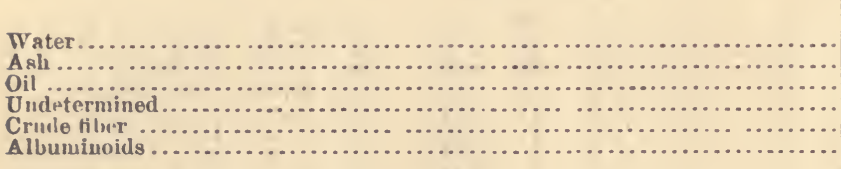 & \begin{tabular}{|r|} 
Per ct. \\
7.71 \\
5.57 \\
.79 \\
62.47 \\
20.83 \\
2.63 \\
\end{tabular} & \begin{tabular}{|r|} 
Per ct. \\
7.83 \\
5.50 \\
.74 \\
63.84 \\
19.64 \\
2.45 \\
\end{tabular} & $\begin{array}{r}\text { Per ct. } \\
8.10 \\
6.22 \\
1.01 \\
60.09 \\
21.45 \\
3.13 \\
\end{array}$ \\
\hline 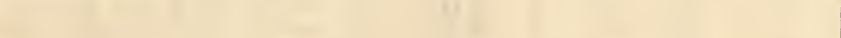 & 100.00 & 100.00 & 100.00 \\
\hline
\end{tabular}

The small number from the West contained rather more albuminoids than the average results for that part of the country, but for the other sectious there is a close agreement. In these samples, oil was determined and found to be extremely small in amount, following the percentage of albuminoidi, the large'st amount of both of these constituents being in the Western hulls, and there seems to be a more or less intimate connection between them.

Of all the cereals this is the richest in oil and albuminoids, the arerage for the former being $\$ .14$ per cent. and for the latter 14.31; of course diminished relatively in the grain as fed, the average composition of which would probably be, as calculated from the average for each portion-

\begin{tabular}{|c|c|c|c|}
\hline . & Kernel. & Hull. & $\begin{array}{l}\text { Whole } \\
\text { grain. }\end{array}$ \\
\hline 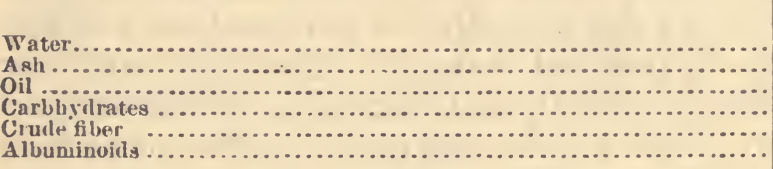 & $\begin{array}{r}\text { Per cent. } \\
4.85 \\
1.50 \\
5.70 \\
46.96 \\
.97 \\
10.02\end{array}$ & \begin{tabular}{|r|} 
Per cent. \\
1.57 \\
1.68 \\
.24 \\
20.41 \\
5.36 \\
.74
\end{tabular} & $\begin{array}{r}\text { Per cent. } \\
6.42 \\
3.18 \\
5.94 \\
67.37 \\
6.33 \\
10.76\end{array}$ \\
\hline f & 70.00 & 30.00 & 100.00 \\
\hline
\end{tabular}

An arerage of 20 analyses of oats in the husk given by Brewer and 153 given by Koenig are given as follows for comparison:

\begin{tabular}{|c|c|c|c|}
\hline 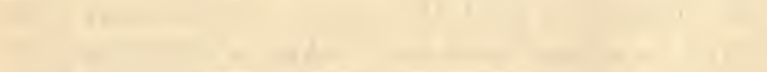 & Brewer. & Koenig. & $\begin{array}{l}\text { Richard- } \\
\text { son. }\end{array}$ \\
\hline 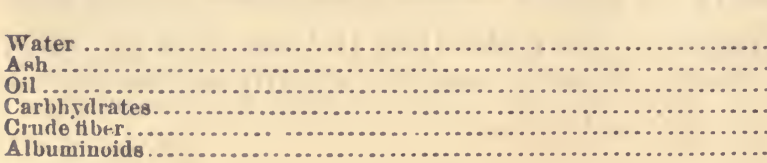 & $\begin{array}{r}\text { Percent. } \\
10.56 \\
2.95 \\
4.97 \\
61.10 \\
9.01 \\
11.41\end{array}$ & $\begin{array}{r}\text { Per cent. } \\
12.37 \\
3.02 \\
5.23 \\
57.78 \\
11.19 \\
10.41\end{array}$ & $\begin{array}{r}\text { Per cent. } \\
6.42 \\
3.18 \\
6.64 \\
66.67 \\
6.33 \\
10.76\end{array}$ \\
\hline
\end{tabular}


The average albuminoids in the grains as fed, calculated in this same manner, is as follows for different sections:

\begin{tabular}{|c|c|}
\hline & Percent. \\
\hline $\begin{array}{l}\text { Northern States .. } \\
\text { Sonthern States... } \\
\text { Paritio: slope...... } \\
\text { All lutine slope.... } \\
\text { Western States... }\end{array}$ & $\begin{array}{r}10.96 \\
10.66 \\
9.611 \\
10.76 \\
11.24\end{array}$ \\
\hline
\end{tabular}

The lowness of the Pacific slope is purely climatic, as has been found to be the case with all the cereals. In appearance the oats from that section are the finest. The fullness of the husk in the Western States or the plumpness of the grain make this the richest in albuminoils as it is fed. The South is poorest for reasons which have been mentioned. That these figures are entirely dependent on the percentage of husk, and not on peculiarities of the kernel, a study of their analyses will show; for, among 179, only 3 fell below 10 per cent. of albuminoids, 4 bolow 11 per cent., and 12 below 12 per cent., while at the same time, only 13 are abure 17 per cent., and 23 above 16 per cent.; that is to say, all but 28, or 81.4 per cent., are within the limits of 12 and 16 per cent., a small variation, although the albuminoids are ligher in amount than wheat; and as the averages for the different States and sections of the country do not vary far from $\mathbf{1 4 . 3}$ per cent., with the exception of the Pacific coast, oats cannot be sail, as far as the grain itself is cencerned, to be in chemical composition very susceptible to their environment, although extremes willely apart are found.

These were as follows:

Extremes in composition of kernels of oals.

\begin{tabular}{|c|c|c|c|c|}
\hline t. & Highest. & State. & Lowest. & State. \\
\hline 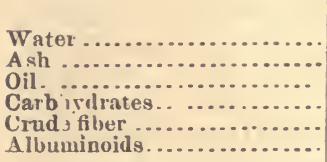 & \begin{tabular}{|r|} 
Per cent. \\
11.13 \\
2.94 \\
11.20 \\
71.91 \\
2.08 \\
19.44
\end{tabular} & 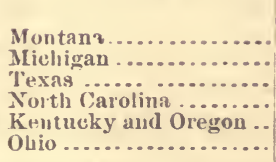 & $\begin{array}{r}\text { Per cent. } \\
4.67 \\
.87 \\
6.50 \\
6.2 .82 \\
.88 \\
9.10\end{array}$ & $\begin{array}{l}\text { Arkansas. } \\
\text { Jowa. } \\
\text { Viıginia. } \\
\text { Yoxas and Missonri. } \\
\text { Ponth Carolina. } \\
\text { Nortl Caruliua. }\end{array}$ \\
\hline
\end{tabular}

The highest percentage of albuminoids was 1.41 per cent. higher than has been found in any other cereal in this country, and the lowest 2 per cent. higher than was found in wheat.

The analysis of the heaviest and largest, of those having the greatest and least weight per busliel, of those having highest and lowest percentage of albuminoids and of the smallest in size and in weight per bushel, have been selected as a study of the effect of these contrasts on the chemicai composition. 
Composition of specimens exhibiting extreme characteristics.

\begin{tabular}{|c|c|c|c|c|c|c|c|c|c|c|c|}
\hline (6) & 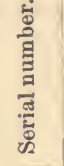 & 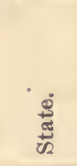 & 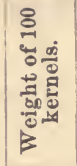 & 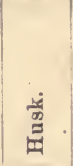 & 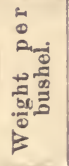 & है & $\frac{\pi}{4}$ & $\ddot{b}$ & $=$ & 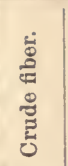 & 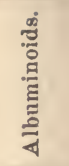 \\
\hline Smallest & 3200 & Nebr. & $\begin{array}{l}G r^{\prime} m s \\
1.512\end{array}$ & $\begin{array}{l}\text { P. ct. } \\
31.21\end{array}$ & $\begin{array}{l}L b s . \\
29.7\end{array}$ & $\begin{aligned} & P . c t \\
& 7.32\end{aligned}$ & $\begin{aligned} & P . c_{e .} \\
& 2.24\end{aligned}$ & $\begin{array}{l}\text { P. ct. } \\
\text { 8. } 72\end{array}$ & $\begin{array}{l}\text { P.ct. } \\
\text { 66. } 39\end{array}$ & $\begin{array}{l}\text { P. ct. } \\
1.33\end{array}$ & $\begin{array}{l}\text { P. ct. } \\
\text { 14. } 00\end{array}$ \\
\hline Largest .......... & 3277 & Oreg. . & 3.786 & 40.85 & 43. 3 & 7.01 & 2. & 7. 87 & 66.80 & 2.07 & 13.83 \\
\hline Cleanest.......... & 3041 & Fla. & 2.880 & 32.87 & 31.5 & 5.83 & 2.52 & 7. 68 & 68.93 & 1. 56 & 13.48 \\
\hline Chaffiest & 3185 & Mis & 2.113 & 25.4 & 38.2 & 7.05 & & 7.81 & $6 \div, 32$ & 1. & 14. \\
\hline Highest per cent. of kernel & 3435 & Wash. & .3. & 20.72 & 43. & 6.55 & 1.55 & 10.57 & 68. 36 & 1. 07 & 11.90 \\
\hline t. of kernel & 339 & Dais & 2.3 & 44. 6 & 38.8 & 8. & & 9.47 & 66.17 & 1.56 & 11.90 \\
\hline Highest albuminoids ....... & 3262 & Ohio . & 2.670 & 39. 17 & 40. & 6. 78 & 2. & 7. 40 & 63.21 & 1.10 & 19.44 \\
\hline Low & 3249 & No & 2.060 & 29.5 & 47. & 7. & & 7.32 & 71. 91 & 1.87 & 9. 10 \\
\hline Heariest weight per & 3020 & Col & 2.958 & 30.24 & 48.8 & 4.80 & $2 .($ & 7. 27 & 66.82 & 1. 00 & 18.03 \\
\hline Lightest weight per bushe & 3002 & & & 31.66 & 24. & & & 8.98 & 66.20 & 1. 20 & 15.23 \\
\hline $\begin{array}{l}\text { Average weight for United } \\
\text { States. }\end{array}$ & & & 2.507 & 30.03 & 37.2 & 6.93 & 2.15 & 8.14 & 67.09 & 1.38 & 14. 31 \\
\hline
\end{tabular}

From the preceding figures nothing can be deduced which shows any such difference as we might expect between the largest and smallest oats. between the cleanest and most chaffy, or between those having the highest and lowest proportion of kernel in the grain. The weight per bushel of the specimen having the lowest percentage of albuminoids is extraordinarily high, while that containing the highest percentage is also above the average. Differences, too, between the weights per bushel of the extreme specimens are in no wis 3 connected with their chemical composition. The largest and one of the finest and heariest oats from Oregon had 1.early the maxim um of husk, and, while the lowest proportion of husk corresponded, of course, with a high weight per bushel, the largest proportion of husks was coincident with a weight per bushel above the average.

An immense number of conditions seem, therefore, to affect the characteristics of this grain, and while in many ways, at first glance, it seems to be less changeable than one would expect, on examination it seems to be quite largely influenced by all the circumstances of its environment, and in a more irregular way than wheat.

Throughout all the averages it will be seen that oats are much drier than other grains, owing largely to their small size. In ash and fiber they are not exceptionable.

Grown in the same locality, under similar conditions, two specimens of different varieties may vary considerably as was found to be the case with wheat. For examples the following determina tions of albuminoids will serve:

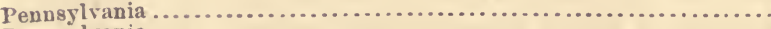

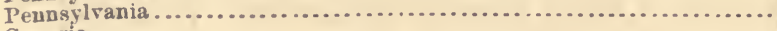

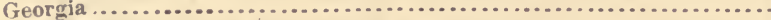

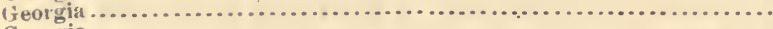

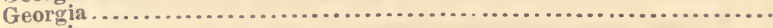

\begin{tabular}{|r|r|} 
& Per cent. \\
\hline $3286_{1}$ & 13.65 \\
$3286_{2}$ & 15.75 \\
$3049_{1}$ & 13.48 \\
$3049_{2}$ & 14.88 \\
$3049_{3}$ & 15.93 \\
$3049_{4}$ & 14.18 \\
$3068_{1}$ & 14.35 \\
$3068_{2}$ & 15.05 \\
$3175_{1}$ & 13.83 \\
$3175_{2}$ & 13.48 \\
\hline
\end{tabular}


(3) The application of nitrogenous nannre in general increased the product decidedly, proportional to the amount of applied nitrogen.

(4) Small or large quantities of pbosphoric acid, together with weak nitrogenous manuring, furnished in the form of Chili saltpeter, showed themselves of paying efficacr.

(5) With strong nitrogenous manuring neither large nor small applications of phosphoric acid bronght abont any action worth mentioning.

(6) The product of grain aud straw was increased in abont equal degrees by the artificial unanring.

(7) The proportion of corn to straw was by thick sowing, on the average 47 to 53 or 1 to 1.13 ; l,y thin sowing, 45 to 55 or 1 to 1.22 .

(8) The harvest sbowed throughont a tolerably low percentage of nitrogen, in the case of the straw ; not, however, an extremely low one, plainly because the rooting 11 p of the weeds and the strengthening of the stems of the oat plant by drilling and harrowing prodnced plants which were, on the average, poor in nitrogen.

(9) By thin sowi!ng the plants were somewhat richer in nitrogen than by thick.

(10) The application of phosploric acid alone was not able to raise the percentage of nitrogen.

(11) On the contrary, the percentage of nitrogen was essentially raised by the application of nitrogenous mannres.

(12) An application of phosplate mannres, together with that of nitrogenous manures, did not alter the percentage of nitrogen.

(13) The greater the harvest the greater also was the percentage of nitrogen in the grain and the straw; from this it appears that the more that was barvested, the better was the quality of the prodnct. A rational method of mannring brings about, not alone greater crops, but also better grain.

(14) The small and poorly-shaped grain harvested with the application of large amounts of nitrogen, and in conseqnence of this somewhat stalled, possess a higher percentage of nitrogen than the fully-developed grain; they cannot, therefore, be looked upon as of less value.

(15) By an application of phosphate manure alone the percentage of oil in the grain was not increased.

(16) On the contrary, by an application of nitrogenous manure alone the oil was decreased.

(1) A weak application of phosphoric acid at the same time with one of nitrogen reproduced the original amount of vil; a stronger application of phosphate even increased il, plaiuly through assistance iu ripening.

(1ช) The grain mannred more strongly with nitrogen was on the whole somewbat richer in fiber and somewhat poorer in nitrogen free uutrients than the grain manured less with nitrogen and more with plosphoric acid.

(19) By a rational method of manuring the albuminoid content of the crop can be alınost donbleri.

(20) In these experiments 55 per cent. of the nitrogen applied in the manures was recovered in the crop.

In his report on the work of the experiment station in 1884, Dr. Maercker continues, in regaril to the investigations:

During this year the same experiments have been carried ont (with oats) again. It is the seconi year of which I here give an account, and the resnlts of the first year are completely confirmed:

(1) That plants relatively poor in nitrogen have been obtained by drilling and harrowing.

(2) That thin sowing has in no case produced as much as thicker sowing.

(3) That a uitrogenons manuring raises strongly the percentage of nitrogen in oats. Further that in this year it has been found that, by manning with phosphoric acid, the allominoids were materially decreased, although the formation of starch has been 
increased. Phosphoric acid hastens the ripening and in general the tendency of the plants to fill out the kernels completely, on which account there is more starch and less protein. Plants relatively poor in nitrogen are therefore produced.

The availability of plant-food is therefore the prime cause of there being so many variations in any one locality corresponding to the soil and manuring on which the crops are dependent.

Our analyses of oats extend over only one year, but Dr. Maercker in two has shown, as our work has with wheat, that "oats appear to be extraordinarily dependent, even in the same locality, in their composition, on the climatic conditions ruling during the opening period." The crops raised in 1882 and 1883, in exactly the same manner, compared in albuminoids, are as follows:

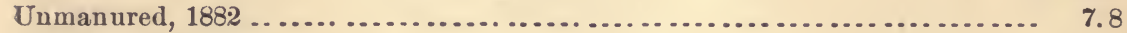

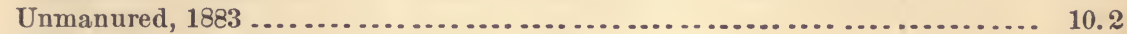

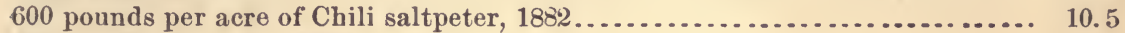

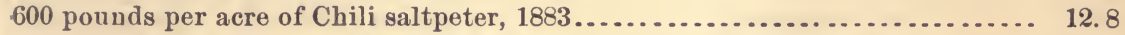

The difference between these figures for the same year illustrates the effect of nitrogenous fertilizers on the percentage of that element in the grain, it being greater in the manured grain by 2.7 per cent. in 1882 and 2.6 per cent. in 1883; and at the same time the effect of the variation in the seasons is as markedly visible.

Comparing the production per acre with the percentage of nitrogen on the grain it was found that those varieties giving the largest yield were poorest in nitrogeu, and the reverse.

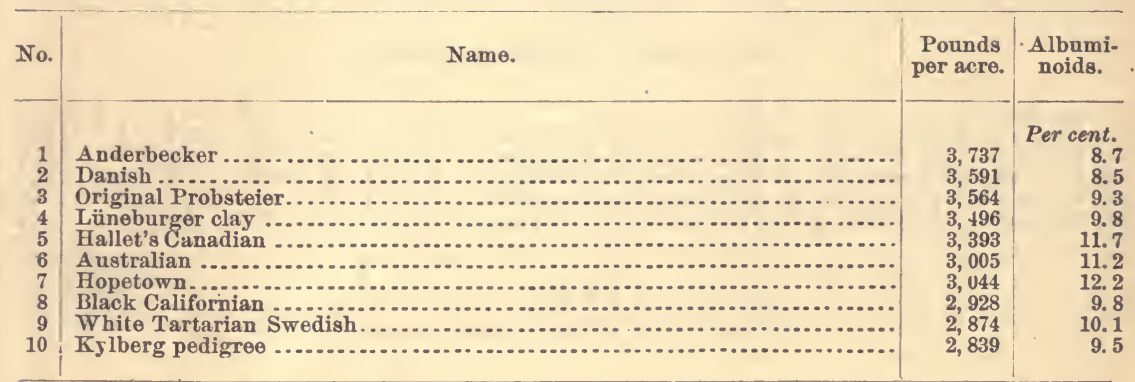

These results, calculated to the amount of nitrogen harvested per acre by the whole plant, explain the differences by showing that all varieties collect about the same amount; consequently, if there is much grain the nitrogen is divided up among it, or if there is much straw the grain is thereby deprived of a certain amount. In 1883 the results were quite different from this. High yields had high percentages of nitrogen, as appears from conclusion 13, previously given. This point, therefore, hardly seems to be entirely settled, but to be largely dependent on the climatic conditions of varying seasons.

For more complete details, reference must be made to the original report upon the experiments, which are models of what should be undertaken in our own country. It is of interest, however, to copy cer- 
tain tables which are of value for comparison with our own analyses and for filling out our knowledge of the plant in directions towards which our investigations did not extend.

\section{EFFECT OF THICK AND THIN SOWING.}

The following tables give the chemical composition of the grain har. vested after thick and thin sowing. The average weight per bushel in both cases was $36.7 \mathrm{lbs}$. All the analyses are calculated to 15 per cent. of water, and the units of nutritive value, being calculated on a German basis, are to us of only relative value.

\section{Composition of crops.}

GRAIN.

[Thick sowing, 44 kilograms per hectare.]

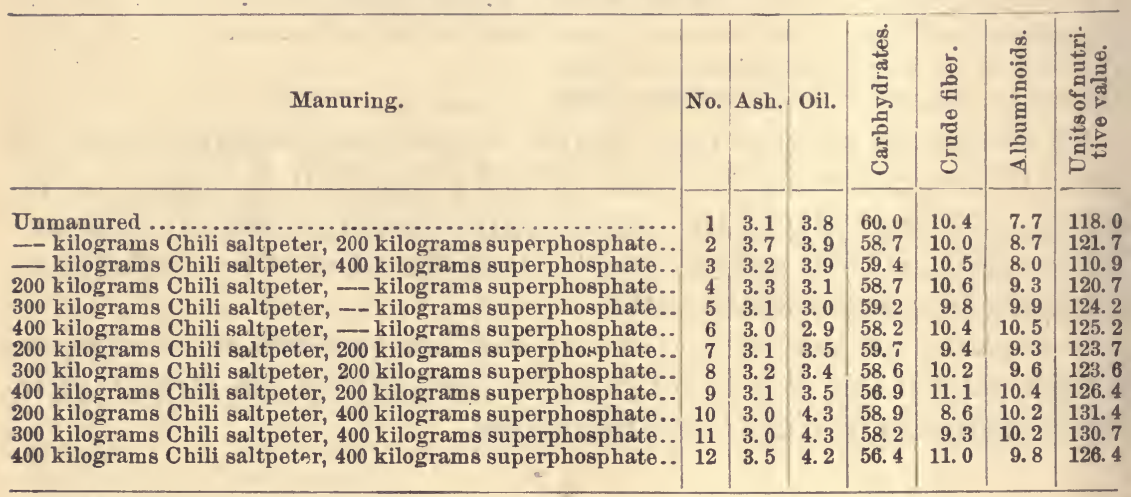

[Thin sowing, 76 kilograms per hectare.]

Unmanured - kilograms Chili saltpeter, 400 kilograms superphosphate.. 14 400 kilograms Chili saltpeter, - kilograms superphosphate.. 15 200 kilograms Chili saltpeter, 200 kilograms superphosphate.. 16 400 kilograms Chili saltpeter, 200 kilograms superphosphate.. 17 400 kilograms Chili saltpeter, 400 kilograms superphosphate.. 18

\begin{tabular}{r|r|r|r|r|r|r}
13 & 3.3 & 3.8 & 59.6 & 10.4 & 7.9 & 118.1 \\
14 & 3.1 & 4.0 & 60.0 & 10.4 & 7.5 & 117.5 \\
15 & 3.3 & 3.1 & 58.8 & 9.9 & 9.9 & 123.8 \\
16 & 3.1 & 3.7 & 59.7 & 9.2 & 9.3 & 124.7 \\
17 & 3.1 & 3.7 & 58.0 & 9.9 & 10.3 & 128.0 \\
18 & 3.1 & 3.6 & 59.3 & 9.6 & 9.4 & 124.3 \\
\hline
\end{tabular}

\section{CHAFF.}

[Thick sowing, 44 kilograms per hectare.]
Unmanured

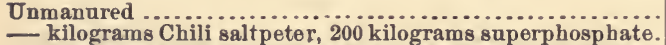
- kilograms Chili saltpeter, 400 kilograms superphosphate. 200 kilograms Chili saltpeter, - kilograms superphosphate. 300 kilograms Chili saltpeter, - kilograms superphosphate. 400 kilograms Chili saltpeter, - kilograms superphosphate. 200 kilograms Chili saltpeter, 200 kilograms superphosphate. 300 kilograms Chili saltpeter, 200 kilograms superphosphate. 400 kilograms Chili saltpeter, 200 kilograms superphosphate. 200 kilograms Chili saltpeter, 400 kilograms superphosphate. 300 kilograms Chili salt peter, 400 kilograms superphosphate. 400 kilograms Chili saltpeter, 400 kilograms superphosphate.

\begin{tabular}{|r|c|c|r|}
1 & 13.9 & $\ldots .$. & 41.9 \\
2 & 14.1 & $\ldots .$. & 41.7 \\
3 & 14.0 & $\ldots$. & 41.8 \\
4 & 14.3 & $\ldots$. & 42.2 \\
5 & 13.6 & $\ldots .$. & 41.3 \\
6 & 15.0 & $\ldots .$. & 40.7 \\
7 & 13.0 & $\ldots .$. & 42.8 \\
8 & 13.7 & $\ldots .$. & 41.8 \\
9 & 15.1 & $\ldots .$. & 40.4 \\
10 & 14.6 & $\ldots .$. & 41.8 \\
11 & 14.2 & $\ldots .$. & 41.9 \\
12 & 13.9 & $\ldots .$. & 42.3
\end{tabular}

\begin{tabular}{|l|}
24.9 \\
24.4 \\
24.9 \\
23.7 \\
24.9 \\
24.0 \\
23.9 \\
24.1 \\
23.6 \\
23.6 \\
23.9 \\
23.1
\end{tabular}

[Thin sowing, 76 kilograms per hectare.]

\begin{tabular}{|c|c|c|c|c|c|c|c|}
\hline Unmanured .... & 13 & 13.8 & & 43.2 & 23.5 & 4.5 & 65.7 \\
\hline - kilograms Chili saltpeter, 400 kilograms superphosphate. & 14 & 14. 1 & & 42. 1 & 23.9 & 4.9 & 66. \\
\hline 400 kilograms Chili saltpeter, - kilograms superphosphate. & 15 & 13. 9 & & 41.6 & 24.2 & 5.3 & 68. \\
\hline 200 kilograms Chili saltpeter, 200 kilograms superphosphate. & 16 & 13.1 & & 43. 8 & 23.5 & 4.6 & 65. \\
\hline 400 kilograms Chili saltpeter, 200 kilograms superphosphate. & 17 & 14.2 & & 42.4 & 24.0 & 4. 4 & 64.4 \\
\hline 400 kilograms Chili saltpeter, 400 kilograms superphosphate. & 18 & 14.4 & & 41.4 & 24.8 & 4.4 & 63.4 \\
\hline
\end{tabular}


Composition of crops-Continued.

\section{STRAW.}

[Thick sowing, 44 kilograms per hectare.]

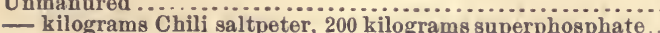
- kilograms Chili saltpeter, 400 kilograms superphosphate. 200 kilograms Chili saltpeter, — kilograms superphosphate. 300 kilograms Chili saltpeter, _ kilograms superphosphate.. 400 kilograms Chili saltpeter, -0 kilograms superphosphate. 200 kilograms Chili saltpeter, 200 kilograms superphosphate.. 300 kilograms Chili saltpeter, 200 kilograms superphosphate. 400 kilograms Chili saltpeter, 200 kilograms superphosphate.. 200 kilograms Chili saltpeter, 400 kilograms superphosphate. 300 kilograms Chili saltpeter, 400 kilograms superphosphate.. 400 kilograms Chili saltpeter, 400 kilograms superphosphate..

\begin{tabular}{|c|c|c|c|c|c|c|}
\hline No. & Ash. & Oil: & 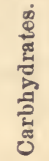 & 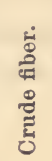 & 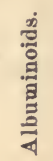 & 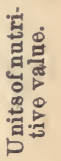 \\
\hline 1 & 5. 7 & .... & 38. 6 & 38.9 & $1 \& 3$ & 48.1 \\
\hline 2 & 5. 9 & & 36.9 & 40.5 & 1. 66 & 45. 4 \\
\hline 3 & 5. 8 & & 39. 2 & 39. 5 & 1. 51 & 46.7 \\
\hline 4 & 5. 9 & & 39. 3 & 38. 2 & 1. 55 & 47.3 \\
\hline 5 & 6.2 & & 37.7 & 39.5 & 1. 62 & 45.7 \\
\hline 6 & 6.2 & ..... & 33. 7 & 43. 2 & 1. 86 & 43.2 \\
\hline 7 & 5. 8 & . & 37.9 & 39.7 & 1. 62 & 45.9 \\
\hline 8 & 6.4 & ..... & 37.1 & 39.8 & 1. 71 & 45.6 \\
\hline 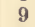 & 6. 1 & & 37.4 & 39.5 & 2. 04 & 47. 4 \\
\hline 10 & 5.9 & . & 36.4 & 41. 2 & 1. 51 & 43.9 \\
\hline 11 & 5.7 & & 36.0 & 41.9 & 1. 36 & 43.0 \\
\hline 12 & 5. 9 & $\ldots$ & 38.4 & 39. 2 & 1. 69 & 46.9 \\
\hline
\end{tabular}

[Thin sowing, 76 kilograms per hectare.]

Unmanured .

- kilograms Chili saltpeter, 400 kilograms superphosphate.

400 kilograms Chili saltpeter, - kilograms superphosphate.. 15

200 kilograms Chili saltpeter, 200 kilograms superphosphate.. 16

400 kilograms Chili saltpeter, 200 kilogi'ams superphosphate.. 17

400 kilograms Chili saltpeter, 400 kilograms superphosphate..

\begin{tabular}{l|l|l|l|l|l|l} 
& 5.5 & $\cdots$ & 38.1 & 40.1 & 1.27 & 44.6 \\
\hline 4 & 5.3 & $\cdots$ & 39.0 & 39.5 & 1.24 & 45.2 \\
6 & 5.4 & $\cdots$ & 37.7 & 40.4 & 1.49 & 45.2 \\
17 & 5.2 & $\cdots$ & 38.2 & 40.3 & 1.27 & 44.7 \\
8 & 5.6 & $\cdots$ & 39.0 & 38.9 & 1.63 & 47.0 \\
\hline & & 37.9 & 40.0 & 1.46 & 45.4
\end{tabular}

COMPOSITION OF THE GRAIN.

\begin{tabular}{|c|c|c|c|c|c|c|c|c|c|}
\hline \multirow[b]{2}{*}{ t } & \multicolumn{3}{|c|}{ Thimsowing. } & \multicolumn{3}{|c|}{ Thick sowing. } & \multicolumn{3}{|c|}{ By Julius Kühn. } \\
\hline & 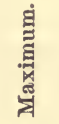 & 起 & 용 & हี & है & ฐึં & 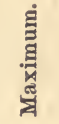 & 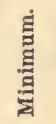 & ్ㅠㅇ \\
\hline 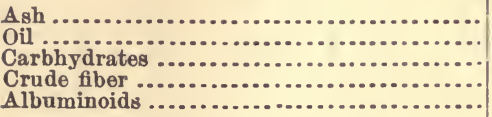 & $\begin{array}{r}\text { 3. } 7 \\
4.3 \\
60.0 \\
11.1 \\
10.5\end{array}$ & $\begin{array}{r}8.0 \\
2.9 \\
56.4 \\
8.6 \\
7.7\end{array}$ & $\begin{array}{r}3.2 \\
3.7 \\
58.6 \\
10.1 \\
9.5\end{array}$ & $\begin{array}{r}3.3 \\
4.0 \\
60.0 \\
10.4 \\
10.3\end{array}$ & $\begin{array}{r}3.1 \\
3.1 \\
58.0 \\
9.2 \\
7.5\end{array}$ & $\begin{array}{r}3.2 \\
3.7 \\
59.3 \\
9.9 \\
9.0\end{array}$ & $\begin{array}{r}2.7 \\
9.2 \\
72.7 \\
16.1 \\
18.5\end{array}$ & $\begin{array}{r}2.7 \\
4.4 \\
48.0 \\
4.1 \\
6.3\end{array}$ & $\begin{array}{r}2.7 \\
6.0 \\
56.6 \\
4.0 \\
12.0\end{array}$ \\
\hline
\end{tabular}

COMPOSITION OF THE CHAFF.

\begin{tabular}{|c|c|c|c|c|c|c|c|c|c|}
\hline $\mathrm{Ash}$. & 15.1 & 13.0 & 14.1 & 13.1 & 13.1 & 13.9 & 11. 0 & 11.0 & 11. 0 \\
\hline 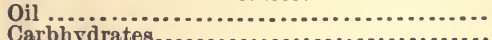 & 42,8 & 40.4 & $\ddot{41.7}$ & $\ddot{43,8}$ & 41.4 & $42 . \overline{5}$ & 43.2 & $\ddot{28.2}$ & $\ddot{37.4}$ \\
\hline Crude fibor. & 24. 9 & 23.1 & 24.1 & 24.8 & 23.5 & 24.0 & 35. 1 & 25.9 & 31.7 \\
\hline 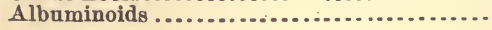 & 5.9 & 4.3 & 5.1 & 5.3 & 4. 4 & 4. 6 & 7.0 & 3.7 & 4. 9 \\
\hline
\end{tabular}

COMPOSITION OF THE STRAW.

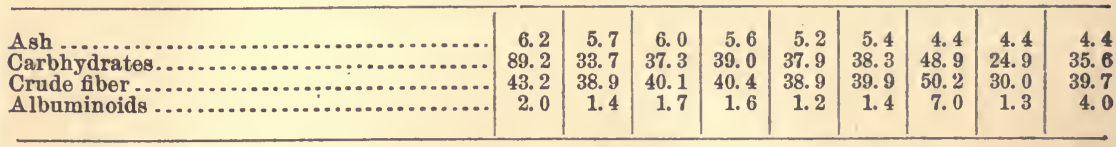


All these results show how variable the oat plant is both for the same year and for different seasons, and that conclusions drawn from the studies of specimens of one season's growth alone may be quite reversed by a wider examination.

Many causes, however, influencing the variations in quality have been explained and the field for future investigation made evident.

\section{RYE.}

Of this cereal, which is of the least importance of any grown in the United States, only 5 samples have been analyzed up to the present time. To supply this deficiency, 56 specimens were collected from the Department correspondents and the principal rye-producing States, at the same time with those of oats and barley.

Their sources were as follows : 


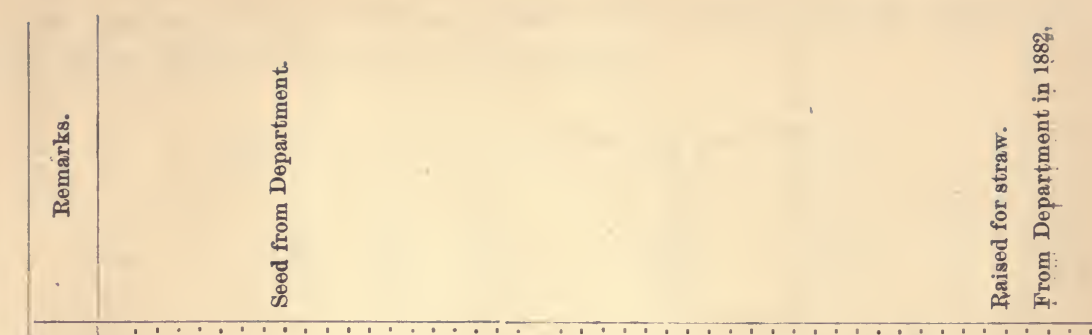

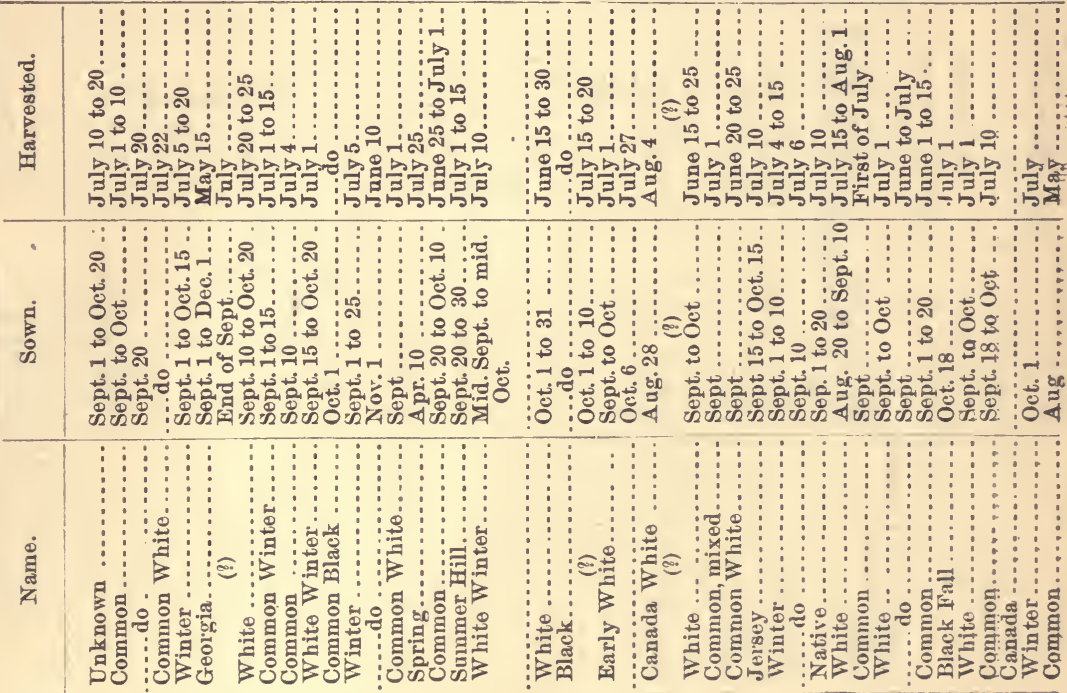

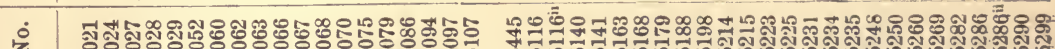

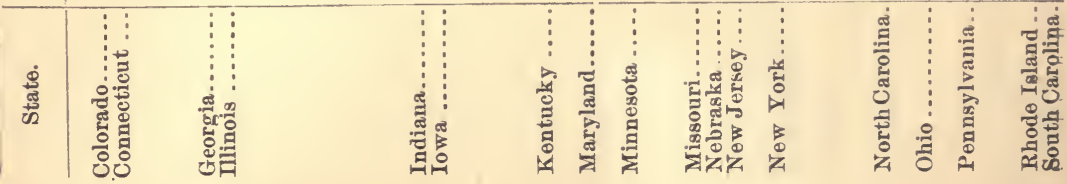


54

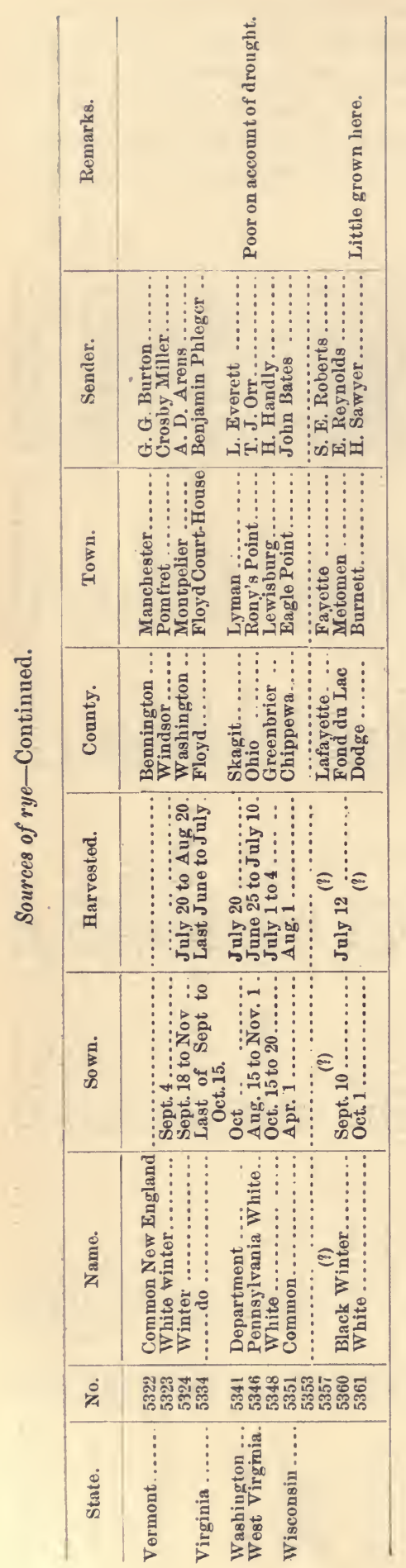


The specimens previously described have been examined physically and chemically with the following results:

Weight of 100 yrains and per bushel of American ryes.

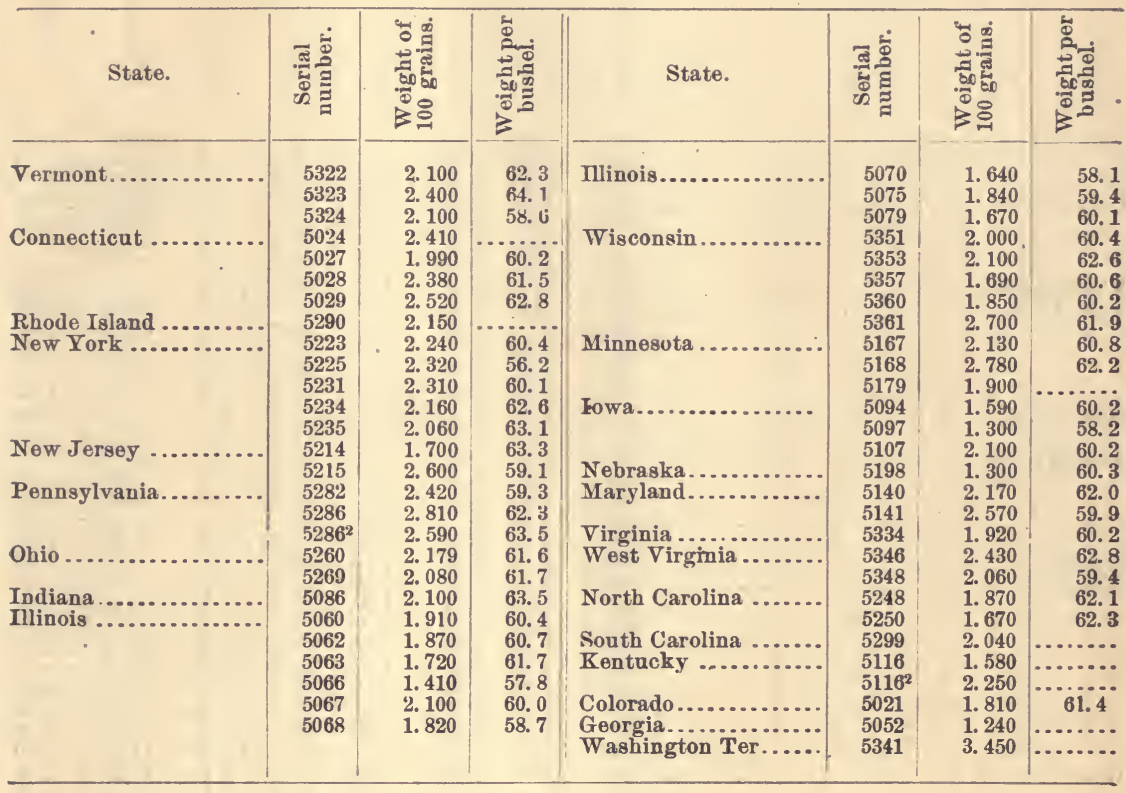

Average weight per bushel and of 100 grains of American ryes.

\begin{tabular}{|c|c|c|c|c|c|c|c|}
\hline State. & 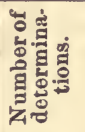 & 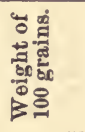 & 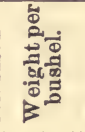 & State. & 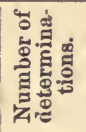 & 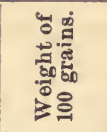 & 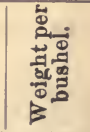 \\
\hline United States. & 56 & 2. 074 & 60.9 & Wisconsin . & 5 & 2.070 & 61.1 \\
\hline Atlantic Slope. & 25 & 2. 189 & 61.2 & Minnesota. & 3 & 2. 270 & 61.5 \\
\hline Northern States ....... & 43 & 2. 074 & 60.8 & Iowa ........ & 3 & 1. 660 & 59.5 \\
\hline Southern States ....... & 11 & 1. 981 & 61.2 & Missouri................. & 1 & & 62.6 \\
\hline Western States........ & 25 & 1. 745 & 60.0 & Nebraska... & 1 & 1.300 & 60.3 \\
\hline Pacific Slope .......... & 2 & 2. 030 & 61.4 & Maryland................ & 2 & 2. 370 & 61.0 \\
\hline Vermont............... & 3 & 2.200 & 61.7 & Virginia .............. & 1 & 1.920 & 60.1 \\
\hline Connecticut ............ & 4 & 2. 320 & 61.5 & West Virginia ........ & 2 & 2. 250 & 61.1 \\
\hline Rhode Island ........... & 1 & 2. 150 & & North Carolina ........ & 2 & 1.770 & 62.2 \\
\hline New York....... & 5 & 2.220 & 60.5 & South Carolina ........ & 1 & 2.040 & ..... \\
\hline New Jersey .... & 2 & 2.150 & 61.2 & Kentucky ............ & 2 & 1. 920 & .......... \\
\hline Pennsylvania .......... & 3 & 2.610 & 61.7 & Georgia............... & 1 & 1.240 & . \\
\hline Ohio .................. & 2 & 2.130 & 61.7 & Colorado.................... & 1 & 1.810 & 61.4 \\
\hline Indiana.................. & 1 & 2. 100 & 63.5 & Washington Ter...... & 1 & 3.450 & ......... \\
\hline Illinois .......... & 9 & 1. 780 & 59.7 & & & te & \\
\hline
\end{tabular}


Composition of American ryes, arranged by States.

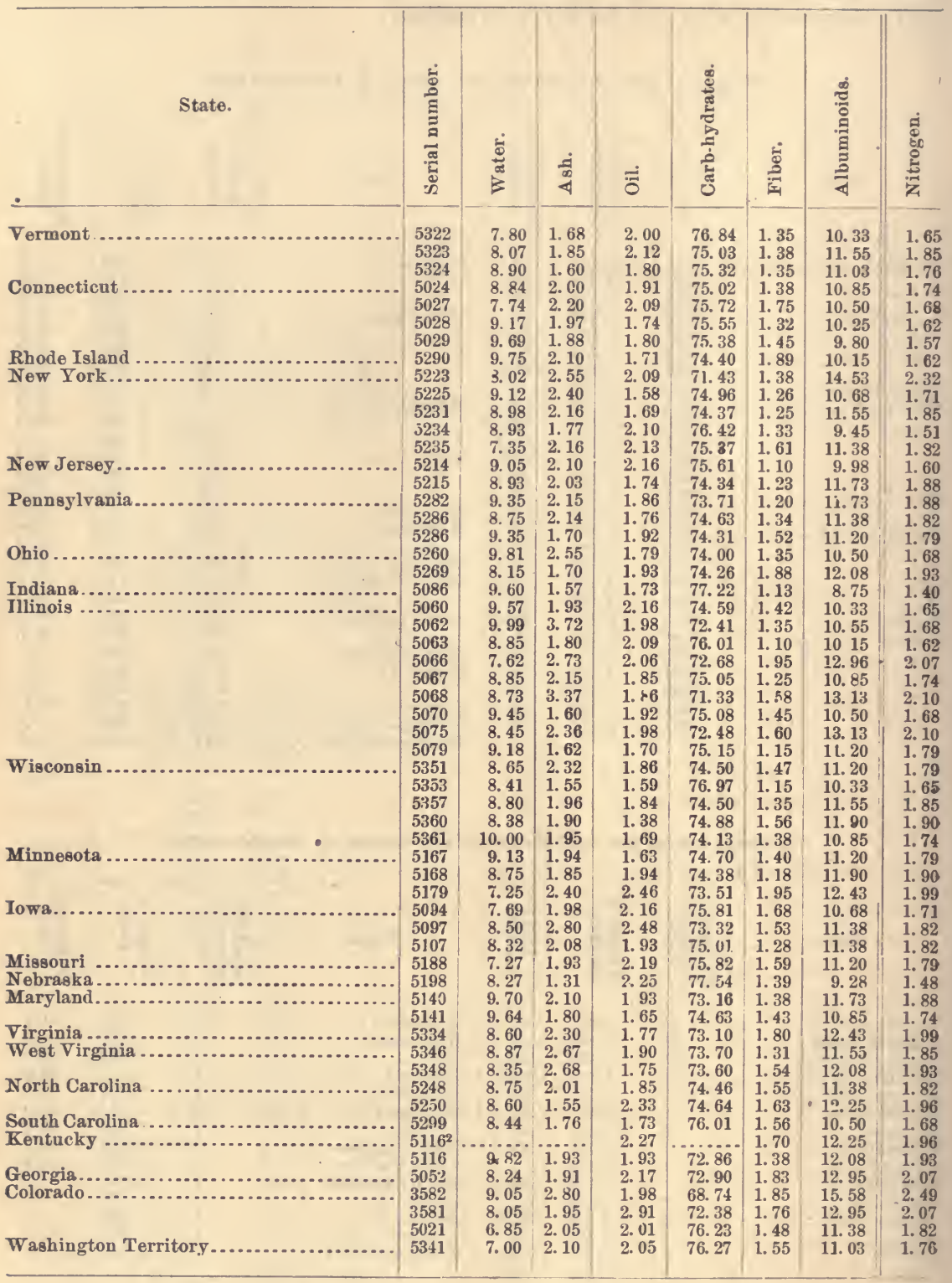


Average composition of American ryes, arranged by States.

\begin{tabular}{|c|c|c|c|c|c|c|c|c|}
\hline · & 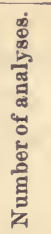 & 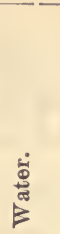 & $\frac{\dot{\pi}}{4}$ & चีं & 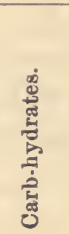 & $\frac{\dot{0}}{\frac{D}{m}}$ & 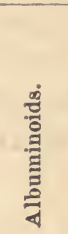 & 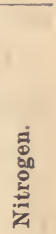 \\
\hline United States. & 57 & 8.67 & 2. 09 & 1.94 & 74.52 & 1.46 & 11.32 & 1.81 \\
\hline Atlantic Slope........ & 25 & 8.75 & 1.99 & 1.91 & 74.74 & 1. 45 & 11.26 & 1.79 \\
\hline 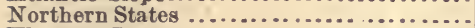 & 43 & 8. 73 & 2. 08 & 1. 92 & 74.74 & 1.43 & 11.10 & 1.79 \\
\hline 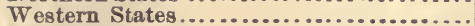 & 25 & 8.71 & 2.12 & 1.94 & 74.62 & 1.44 & 11.17 & 1. 79 \\
\hline Southern States.............. & 10 & 8.80 & 2.07 & 1.90 & 74.01 & 1.54 & 11.68 & 1.88 \\
\hline Pacific Slope................. & 4 & 7. 74 & 2.23 & 2.24 & 73.40 & 1.66 & 12.73 & 2.04 \\
\hline Vermont & 3 & 8.26 & 1. 71 & 1.97 & 75.73 & 1.36 & 10.97 & 1.75 \\
\hline Connecticut & 4 & 8.86 & 2.01 & 1.88 & 75.41 & 1.48 & 10.33 & 1.65 \\
\hline Rhode Island ... & 1 & 9. 75 & 2.10 & 1.71 & 74.44 & 1.89 & 10.15 & 1.62 \\
\hline New York...... & 5 & 8.48 & 2.21 & 1.92 & 74.51 & 1.36 & 11.52 & 1. 84 \\
\hline New.Jersey. & 2 & 8.99 & 2. 06 & 1.95 & 74.98 & 1.16 & 10.86 & 1. 74 \\
\hline Pennsylvania. & 3 & 9.15 & 1.99 & 1.85 & 74.22 & 1.35 & 11.44 & 1.83 \\
\hline Ohio ............ & 2 & 8.98 & 2.13 & 1.86 & 74.13 & 1. 61 & 11.29 & 1.81 \\
\hline Indiana & 1 & 9.60 & 1.57 & 1.73 & -7.22 & 1.13 & 8.75 & 1.40 \\
\hline Illinois ................. & 9 & 8.96 & 2. 36 & 1.96 & 73.87 & 1.43 & 11.42 & 1.83 \\
\hline $\mathrm{W}$ isconsin .... & 5 & 8.85 & 1.94 & 1.67 & 74. 99 & 1.38 & 11.17 & 1.79 \\
\hline Minnesota..... & 3 & 8. 36 & 2. 06 & 2. 01 & 74.20 & 1. 51 & 11.84 & 1.89 \\
\hline Iowa........... & 3 & 8. 17 & 2.29 & 2.19 & 74.71 & 1.50 & 11.14 & 1.78 \\
\hline Missouri....... & 1 & 7.27 & 1.93 & 2.19 & 75.82 & 1. 59 & 11. 20 & 1. 79 \\
\hline Nebraska..... & 1 & 8.27 & 1.31 & 2.25 & 77.54 & 1.35 & 9.28 & 1.48 \\
\hline Maryland ............... & 2 & 9.67 & 1.95 & 1. 79 & 73.90 & 1.40 & 11.29 & 1. 81 \\
\hline Virginia .... & 1 & 8.60 & 2.30 & 1. 77 & 73.10 & 1.80 & 12.43 & 1.99 \\
\hline West Virginia. & 2 & 8.61 & 2. 67 & 1.83 & 73.65 & 1. 42 & 11.82 & 1.89 \\
\hline North Carolina ... & 2 & 8.17 & 1.78 & 2.09 & 74.55 & 1.59 & 11.82 & 1.89 \\
\hline South Carolina ..... & 1 & 8.44 & 1.76 & 1.73 & 76.01 & 1.56 & 10.50 & 1. 68 \\
\hline Kentucky ...... & 1 & 9.82 & 1.93 & 1.93 & 72.86 & 1. 38 & 12. 08 & 1.93 \\
\hline Georgia........ & 1 & 8.24 & 1.91 & 2.17 & 73. 90 & 1.83 & 11.35 & 2. 07 \\
\hline Colorado....... & 3 & 7.98 & 2.24 & 2. 30 & 72.45 & 1.70 & 13. 20 & 2.13 \\
\hline Washington Territory. & 1 & 7.00 & 2.10 & 2. 05 & 76.27 & 1.55 & 11.03 & 1.76 \\
\hline
\end{tabular}

The largest specimen was from Washington Territory, weighing 3.450 grams, the next from Minnesota, weighing 2.780 per 100 grains, and the heaviest weight per bushel from Vermont, 64.1 pounds. The smallest were from Iowa and Nebraska, weighing 1.300 grams per hundred, and the lightest from New York, 56.2 pounds per bushel, the average for the country being 2.074 and 60.9. The largest and heaviest ryes were found on the Atlantic coast and in the Northern States. The Pacific slope was not well represented.

The average weight per bushel is much higher than is usually accepted for'rye, but the specimens in hand certainly reached those figures perhaps being very clean or selected samples above the average production. Illinois, which in the last census year produced more of the crop than any State except New York, sends the smallest and the lightest average grain.

In chemical composition the following extremes were found :

\begin{tabular}{|c|c|c|c|c|}
\hline f & Highest. & State. & Lowest. & State. \\
\hline 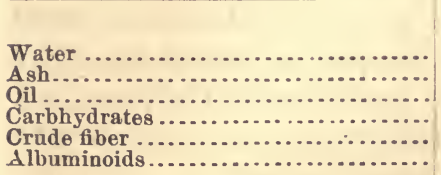 & $\begin{array}{r}\text { Per cent. } \\
10.00 \\
3.72 \\
2.91 \\
77.54 \\
1.90 \\
15.58\end{array}$ & $\begin{array}{l}\text { Wisconsin ..... } \\
\text { Illinois ....... } \\
\text { Colorado...... } \\
\text { Nebraska.... } \\
\text { Minnesota.... } \\
\text { Colorado ...... }\end{array}$ & $\begin{array}{r}\text { Per cent. } \\
7.00 \\
1.31 \\
1.38 \\
68.74 \\
1.10 \\
8.75\end{array}$ & $\begin{array}{l}\text { Washington Territory. } \\
\text { Nebraska. } \\
\text { Wisconsin. } \\
\text { Colorado. } \\
\text { Illinois. } \\
\text { Indiana. }\end{array}$ \\
\hline
\end{tabular}


But 5 were below 10 per cent. of albuminoids, and all but 4 were below 13 per cent.

The grain cannot be said to be extremely variable. The averages for the country is here given, together with an average of 49 analyses of ryes from all sources given by Koenig:

\begin{tabular}{|c|c|c|}
\hline & $\begin{array}{l}\text { United } \\
\text { States. }\end{array}$ & Koenig. \\
\hline 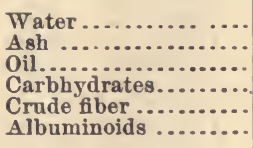 & $\begin{array}{r}8.67 \\
2.09 \\
1.94 \\
74.52 \\
1.46 \\
11.32\end{array}$ & $\begin{array}{r}15.06 \\
1.89 \\
1.79 \\
67.81 \\
2.01 \\
11.52\end{array}$ \\
\hline
\end{tabular}

The extremes of albuminoid in Koenig's analyses were 16.93 and 7.91 per cent., which is wider than among our specimens. The difference between our grain and that of the Continent appears in the greater moisture of the latter, as is to be expected, together with more ash and oil and less fiber. For different parts of the United States the averages are very nearly concordant, the only variation being the difference of half a per cent. albuminoids and a little more fiber in ten specimens from the South. The nitrogenous constituents are practically the same. This cereal is richer than corn in this element, and not quite so rich as wheat.

Rye cannot be considered as being very susceptible to climatic conditions; in fact, it will flourish where other cereals will not. It requires therefore no greater care in its improrement than the selection of the variety giving the largest yield, and careful cultivation.

\section{BARLEY.}

Of American barley, from any point of view, but little has been known hitherto. Until lately, only nine chemical anaylses have been made, and, as Professor Brewer remarks, these are too few in number for generalizations. Statistics show that we have not produced enough of the cereal to supply the demand, and that it is always necessary to import a large amount every year. A study, therefore, of the conditions which affect the production of barley in the United States, which portions produce the most valuable grain, and how the composition varies in different localities as the result of climate and general environment, will be of interest, as showing the possibilities and best localities for the extension of the growth of this cereal.

Before discussing the results of our examination of the numerous American specimens collected through our agents, it will be of interest to give abstracts of some investigations on the production of barley in certain portions of Germany and this country, showing the yield, weight, physical characters and composition, and the directions in which it is considered desirable that this grain shonld be developed. 
Dr. Maercker, of Halle, in a report on "Barley Experiments with Seed from Various Sources," a copy of which he has been good enough to send us, says that the problem of the production of the best barley has become an important one in the last few years in the province of the Salle, which has heretofore produced the best quality, but recently has met with much ill luck. To the end of studying the conditions affecting this cereal and learning the physical and chemical characteristics of the best varieties, seed selected by a mixed jury from a large exhibition of barleys were devoted to the experiment, and distributed among the leading agriculturists of the province. The varieties were grown with different supplies of nitrogenous manures, all the seed having been judged extremely fine (hochfein), and found to possess the following characteristics :

\begin{tabular}{|c|c|c|}
\hline 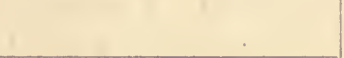 & Albuminoids. & Mealy kernels. \\
\hline 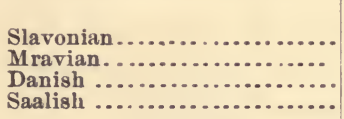 & $\begin{array}{l}\text { Per cent. } \\
\text { 7. } 7 \\
7.7 \\
7.7 \\
8.1\end{array}$ & $\begin{array}{l}\text { Per cent. } \\
92 \\
90 \\
90 \\
80\end{array}$ \\
\hline
\end{tabular}

From the experiments it was found that in the matter of yield the higher was obtained with the larger supply of nitrogenous manure, but that the quality was somewhat injured thereby, as the percentage of albuminoids was considerably raised, as can be seen from the determinations which were made:

\begin{tabular}{|c|c|c|c|c|}
\hline 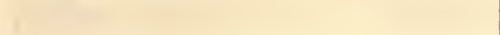 & Saalish. & Danish. & Moravian. & Slavonian. \\
\hline $\begin{array}{l}\text { Original seed . Chili saitpeter, per hectare..... } \\
100 \text { kilograms } \\
200 \text { kilograms Chili saltpeter, per hectare.... }\end{array}$ & $\begin{array}{r}8.10 \\
8.19 \\
8.48\end{array}$ & $\begin{array}{l}7.70 \\
9.16 \\
9.56\end{array}$ & $\begin{array}{l}7.70 \\
9.18 \\
9.78\end{array}$ & $\begin{array}{l}7.70 \\
8.92 \\
9.52\end{array}$ \\
\hline
\end{tabular}

The quality or consistency of the original seed was found in most cases to be lowered; and although the Slowakisch barley was superior to the rest, three samples out of seventeen being extremely fine, three fine, and eight good, it was nevertheless apparent that although the quality of the seed is an essential factor in the quality of the harvest, it is not the only one, but that climate, soil, manuring, and cultivation are much more important and of greater influence. One can in no way expect that the production of barley can be improved by selected seed alone. Care in other directions and favorable climatic influences, orer which we have no control, are necessary as well.

The heavy manuring of nitrogenous material, as has been said, in. jured the quality, and how much so in comparison with a light one can be seen by enumerating the number of experimental samples which were found to be below the mean in quality. Of 89 manured with 100 kilograms of Chili saltpeter per hectare, only 6 were below medium; of 
78 having 200 kilograms per hectare, 16 were below medium. Nitrogenous manures are not, accordingly, to be considered advisable on barley.

As to the relation of percentage of albuminoids, weight per bushel, and consistency to the quality of the grain, Dr. Mäercker remarks :

For a long time the author has busied himself with the question whether the amount of albuminoids stood in any relation to the value of barley, and in many cases this question could be answered that with few exceptions a barley rich in albuminoids is of poor quality, while a low content of albuminoids in general was an expression of high quality. It is, of course, understood that exterior conditions, rain, moisture, \&c., ean injure their value and make the barleys of low albuminoids of less worth than others richer in nitrogen. In proof of this, the decisions of the judges and the testimony of the laboratory furnish all that is desired.

The albuminoids in the crop as given in the previous table were over 1 per cent. higher than in the seed, and the quality was adjudged correspondingly poorer, and the specimen considered to be the worst was found to have increased 2.08 per cent. orer the seed. Comparing the decision of the judges with the percentages of albuminoids the following coincidence was found:

\begin{tabular}{|c|c|}
\hline Specimens denominated- & $\begin{array}{l}\text { Mean per } \\
\text { cent. of al. } \\
\text { buminoids. }\end{array}$ \\
\hline 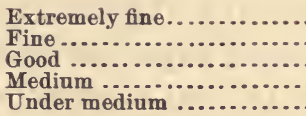 & $\begin{array}{r}8.09 \\
8.67 \\
8.93 \\
9.78 \\
10.24\end{array}$ \\
\hline
\end{tabular}

From these figures it cannot be denied "that high content of albuminoids appears to be incompatible with high quality."

To the weight the judges paid little attention. The determinations showed no relation between quality and weight.

Weight per hectoliter in kilograms* of the seed was as follows:

\begin{tabular}{|c|c|}
\hline w & Per cent. \\
\hline $\begin{array}{l}\text { Slavon } \\
\text { Morav }\end{array}$ & \\
\hline 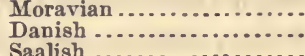 & $\begin{array}{l}70.8 \\
69.0\end{array}$ \\
\hline
\end{tabular}

And of the crops in the mean:

\begin{tabular}{|c|c|c|c|c|}
\hline$x^{2}$ & Saalish. & Danish. & Moravian. & Slavonian. \\
\hline 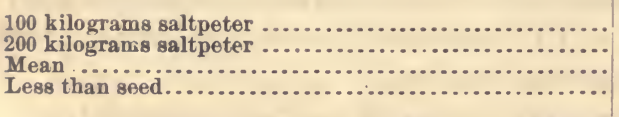 & $\begin{array}{r}67.2 \\
67.2 \\
67.1 \\
2.1\end{array}$ & $\begin{array}{r}67.2 \\
66.8 \\
67.0 \\
2.0\end{array}$ & $\begin{array}{r}66.6 \\
67.5 \\
67.1 \\
3.7\end{array}$ & $\begin{array}{r}67.3 \\
66.7 \\
67.0 \\
1.7\end{array}$ \\
\hline
\end{tabular}

The weight of the crop is on the average less than the seed; but between the different varieties there is no difference in the mean weight, despite the fact that there is a difference in quality.

\footnotetext{
* Kilogram per hectoliter $\times .775 \%=$ pounds per bushel.
} 
In regard to the mealy consistency of the grain the following figures furnish an explanation:

Per cent. of mealy kernels.

\begin{tabular}{|c|c|c|c|c|}
\hline 1 & Saalish. & Danish. & Moravian & $\begin{array}{l}\text { Slavon- } \\
\text { ian. }\end{array}$ \\
\hline 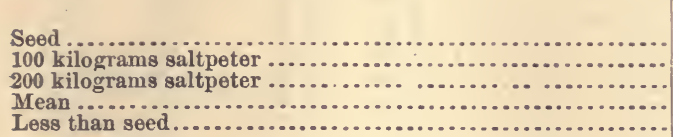 & $\begin{array}{r}\text { Per cent. } \\
80.0 \\
62.4 \\
64.9 \\
63.7 \\
16.3\end{array}$ & $\begin{array}{r}\text { Per cent. } \\
90.0 \\
70.1 \\
65.9 \\
68.0 \\
22.0\end{array}$ & \begin{tabular}{|r|} 
Per cent. \\
90.0 \\
68.7 \\
66.8 \\
67.8 \\
22.2
\end{tabular} & $\begin{array}{r}\text { Per cent. } \\
92.0 \\
77.5 \\
64.7 \\
71.1 \\
20.9\end{array}$ \\
\hline
\end{tabular}

The mealiness of the crop is much less than of the seed, which agrees again with the decision of the judges, who it may be remarked placed the greatest dependence on the consistency of the kernel in forming their opinion, and in other respects with the conclusions derived from other characteristics.

Among a collection of 50 barleys which were submitted with the experimental specimeus already mentioned, there were found none worthy of mention except the crop of one gentleman who had used no nitrogen but heavy manuring with phosphoric acid. His barleys were graded as follows :

\begin{tabular}{|c|c|c|c|c|c|}
\hline 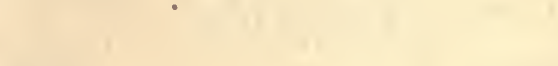 & $\begin{array}{c}\text { Extra } \\
\text { fine }(a) .\end{array}$ & $\begin{array}{c}\text { Extra } \\
\text { fine }(b) .\end{array}$ & $\begin{array}{c}\text { Extra } \\
\text { fine }(c) .\end{array}$ & $\begin{array}{c}\text { Extra } \\
\text { fine }(d) .\end{array}$ & Fine. \\
\hline 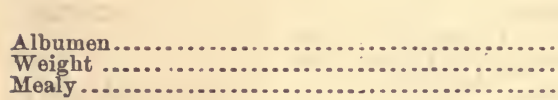 & $\begin{array}{r}\text { Per cent. } \\
80.8 \\
70.0 \\
88.0\end{array}$ & $\begin{array}{r}\text { Per cent. } \\
79.9 \\
69.1 \\
88.0\end{array}$ & $\begin{array}{r}\text { Per cent. } \\
7.7 \\
68.1 \\
82.0\end{array}$ & $\begin{array}{r}\text { Per cent. } \\
80.4 \\
70.3 \\
86.0\end{array}$ & $\begin{array}{r}\text { Per cent. } \\
8.2 \\
67.3 \\
86.0\end{array}$ \\
\hline
\end{tabular}

From the preceding experiments we learn that the characteristics of a first quality barley are its consistency, color, and its albuminoid per. centage, the latter in fine barleys not exceeding 8.67.

Several other investigators in previous years have not found the average up to the standard which has been set by the judges just mentioned. The results of Reischauer* show that the barleys which he had in hand were somewhat richer in nitrogen than those of Mäercker.

In 100 parts of dry substance.

\begin{tabular}{|c|c|c|c|c|c|c|c|}
\hline F & Nitrogen. & $\begin{array}{c}\mathrm{N} \times 6.25 \\
\text { Albumi. } \\
\text { noids. }\end{array}$ & Ash. & $\begin{array}{l}\text { Phos- } \\
\text { phoric } \\
\text { acid. }\end{array}$ & Silica. & $\begin{array}{l}\text { Iron } \\
\text { oxide. }\end{array}$ & Lime. \\
\hline $\begin{array}{l}\text { Maximum } \\
\text { Minimum }\end{array}$ & $\begin{array}{r}\text { Per cent. } \\
2.856 \\
1.282\end{array}$ & $\begin{array}{c}\text { Per cent. } \\
17.85 \\
8.01\end{array}$ & $\begin{array}{c}\text { Per cent. } \\
\text { 3. } 34 \\
\text { 2. } 12\end{array}$ & $\begin{array}{r}\text { Per cent. } \\
1.145 \\
0.614\end{array}$ & $\begin{array}{r}\text { Per cent. } \\
0.845 \\
0.460\end{array}$ & $\begin{array}{r}\text { Per cent. } \\
0.0694 \\
0.0019\end{array}$ & $\begin{array}{r}\text { Per cent. } \\
0.151 \\
0.043\end{array}$ \\
\hline Average .............. & 1.729 & 10.804 & 2. 799 & 0.902 & 0.641 & 0.0200 & 0.068 \\
\hline
\end{tabular}

* Zeitschrift für das gesamte Brauwesen, 353-363; Bied. Centralblatt 11, 42-43. 
In 100 parts of dry substance-Continued.

\begin{tabular}{|c|c|c|c|c|}
\hline Source of barley. & Nitrogen. & $\begin{array}{c}\mathrm{N} \times 6.25 \\
\text { Albumi- } \\
\text { noid. }\end{array}$ & Ash. & $\begin{array}{l}\text { Phos- } \\
\text { phoric } \\
\text { acid. }\end{array}$ \\
\hline 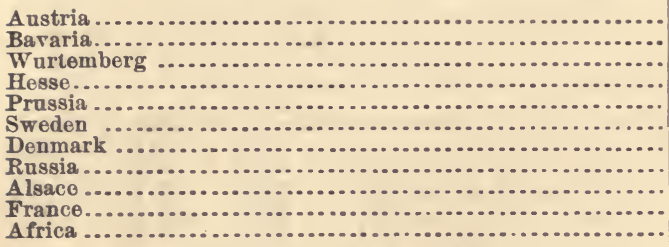 & $\begin{array}{l}1.564 \\
1.655 \\
1.658 \\
1.750 \\
1.806 \\
2.121 \\
1.661 \\
2.188 \\
1.699 \\
1.769 \\
1.833\end{array}$ & $\begin{array}{r}9.77 \\
10.34 \\
10.36 \\
10.93 \\
11.29 \\
12.36 \\
10.38 \\
13.67 \\
10.62 \\
11.05 \\
11.46\end{array}$ & $\begin{array}{l}2.818 \\
2.848 \\
2.860 \\
2.923 \\
2.853 \\
2.515 \\
2.720 \\
2.753 \\
2.802 \\
2.936 \\
2.730\end{array}$ & $\begin{array}{l}0.900 \\
0.944 \\
0.962 \\
1.019 \\
0.920 \\
0.841 \\
0.928 \\
0.921 \\
0.880 \\
0.897 \\
0.781\end{array}$ \\
\hline
\end{tabular}

Louis Marx has also examined a large number of barleys-four hun. dred-from various countries, extending over six years' crops. His results have furnished the following averages for the amount of albuminoids usually present:

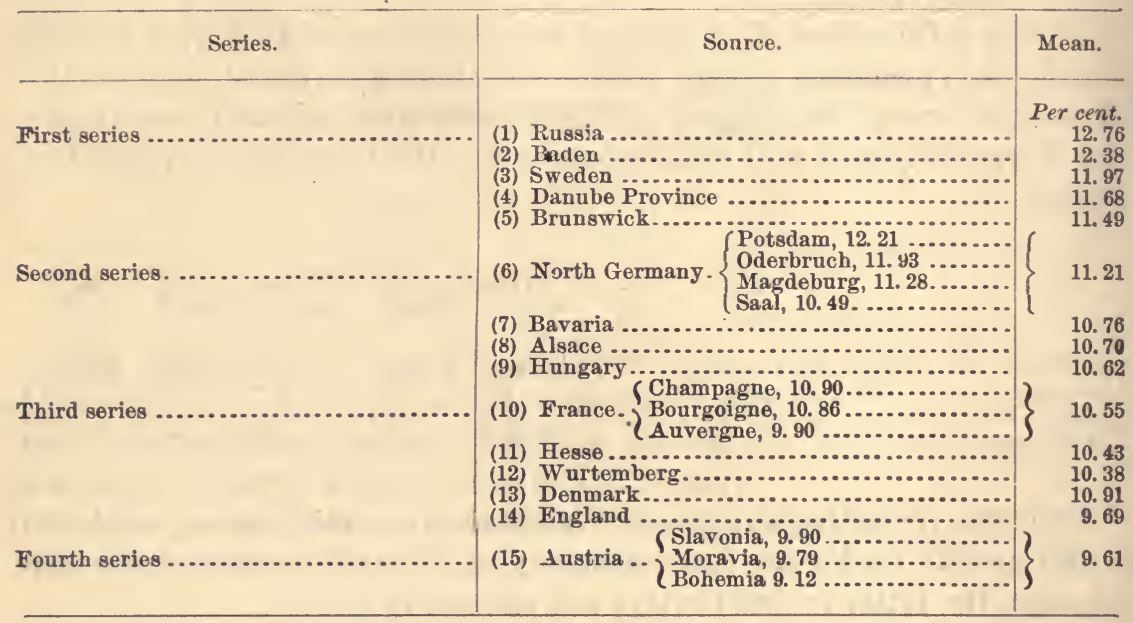

In Russia, as with wheats, barley was found to be rich in albuminoids, one reaching 16.00. Bohemia and England, both celebrated for their malt, furnished but few samples with over 10.00 per cent. Bavaria, with 68 samples, had only 6 over 12.00 per cent.

The thick-hulled barleys were as a whole poorer in nitrogen, the hull being, of course, poor in that element. There was found to be no relation between nitrogen and phosphoric acid.

Some analyses by Lunter of barleys of the crop of 1883, used in the experimental brewery at Munich, have been published lately in Biedermann's Centralblatt fuir Agrikulturchemie, without great comment.

$\mathrm{He}$ finds in the experimental field that continuous cultivation for years can be carried on without essential alteration of the quality. 


\begin{tabular}{|c|c|c|c|c|c|c|c|}
\hline \multirow[b]{2}{*}{ 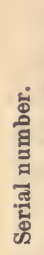 } & \multirow{2}{*}{ Source of barley. } & \multicolumn{6}{|c|}{ Dry substances. } \\
\hline & & 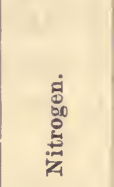 & 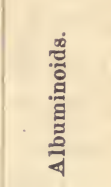 & 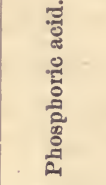 & 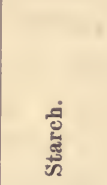 & है & 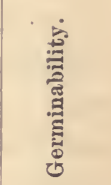 \\
\hline $\begin{array}{l}1 \\
2 \\
3 \\
4 \\
5 \\
6 \\
7 \\
8\end{array}$ & 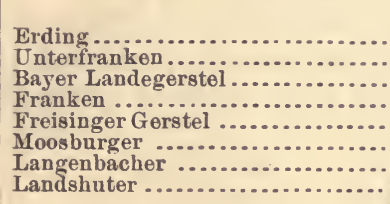 & $\begin{array}{r}\text { Per cent. } \\
1.646 \\
1.806 \\
1.661 \\
1.601 \\
1.623 \\
1.585 \\
1.680 \\
1.722\end{array}$ & $\begin{array}{r}\text { Per cent. } \\
10.29 \\
11.29 \\
10.38 \\
10.00 \\
11.14 \\
9.90 \\
10.50 \\
10.76\end{array}$ & $\begin{array}{r}\text { Per cent. } \\
1.003 \\
0.931 \\
1.047 \\
0.913 \\
0.951 \\
0.930 \\
0.935 \\
1.034\end{array}$ & $\begin{array}{r}\text { Per cent. } \\
71.28 \\
59.62 \\
66.45 \\
66.61 \\
65.84 \\
65.16 \\
65.82 \\
64.18\end{array}$ & $\begin{array}{r}\text { Per cent. } \\
8.46 \\
17.84 \\
16.58 \\
14.82 \\
12.28 \\
12.47 \\
12.67 \\
13.26\end{array}$ & $\begin{array}{r}\text { Per cent. } \\
30.80 \\
88.60 \\
90.10 \\
95.40 \\
80.15 \\
83.14 \\
90.00 \\
89.20\end{array}$ \\
\hline
\end{tabular}

These samples, in albuminoids, certainly do not attain the high standard of quality sèt by Maercker.

Of American barleys, the only investigation, in addition to nine analyses collected by Professor Brewer, is that of eleven specimens at the Brewers' Experiment Station in New York, in 1883 or 1884, * the results of which are here given in one hundred parts of dry substance:

\begin{tabular}{|c|c|c|c|c|c|c|c|c|}
\hline \multirow[b]{2}{*}{ 离 } & \multirow[b]{2}{*}{ Source. } & \multirow{2}{*}{ 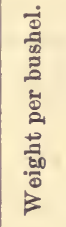 } & \multirow[b]{2}{*}{ 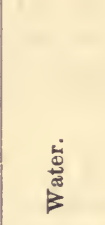 } & \multirow[b]{2}{*}{ 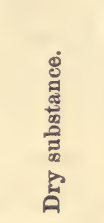 } & \multicolumn{4}{|c|}{ Dry substanca } \\
\hline & & & & & 通 & 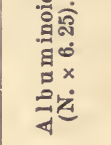 & मีं & 贾 \\
\hline $\begin{array}{l}1 \\
2 \\
3 \\
4 \\
4 \\
5 \\
6\end{array}$ & 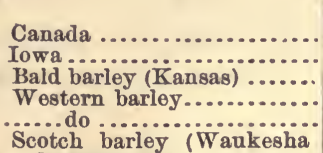 & $\begin{array}{l}L b 8 . \\
50 \frac{1}{2} \\
48 \frac{3}{4} \\
57 \frac{1}{2} \\
48 \frac{1}{2} \\
48 \frac{1}{2}\end{array}$ & $\begin{array}{r}\text { Percent. } \\
10.04 \\
9.22 \\
10.41 \\
9.56 \\
9.36\end{array}$ & $\begin{array}{r}\text { Per cent. } \\
89.96 \\
90.78 \\
89.59 \\
90.44 \\
90.64\end{array}$ & $\begin{array}{r}\text { Per cent. } \\
63.63 \\
59.48 \\
64.49 \\
60.30 \\
61.36\end{array}$ & $\begin{array}{r}\text { Per cent. } \\
10.73 \\
11.18 \\
10.16 \\
12.39 \\
11.36\end{array}$ & $\begin{array}{r}\text { Per cent. } \\
2.78 \\
3.16 \\
2.86 \\
3.21 \\
3.31\end{array}$ & $\begin{array}{r}\text { Pcr cent. } \\
0.950 \\
1.149 \\
0.997 \\
1.124 \\
1.278\end{array}$ \\
\hline $\begin{array}{r}7 \\
8 \\
9\end{array}$ & 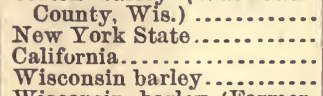 & \begin{tabular}{c}
48 \\
\hdashline 54 \\
$48 \frac{1}{2}$
\end{tabular} & $\begin{array}{l}10.21 \\
12.05 \\
12.40 \\
11.89\end{array}$ & $\begin{array}{l}89.79 \\
87.95 \\
87.60 \\
88.11\end{array}$ & $\begin{array}{l}59.54 \\
66.31 \\
66.54 \\
65.98\end{array}$ & $\begin{array}{r}8.18 \\
12.79 \\
13.60 \\
10.27\end{array}$ & $\begin{array}{l}3.77 \\
2.59 \\
2.45 \\
2.84\end{array}$ & $\begin{array}{r}1.582 \\
\cdots \\
1.000\end{array}$ \\
\hline $\begin{array}{l}10 \\
11\end{array}$ & 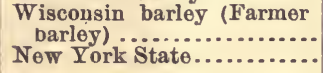 & $\begin{array}{l}47 \\
50\end{array}$ & $\begin{array}{l}11.56 \\
14.06\end{array}$ & $\begin{array}{l}88.44 \\
85.94\end{array}$ & $\begin{array}{l}66.29 \\
63.70\end{array}$ & $\begin{array}{l}12.23 \\
11.62\end{array}$ & $\begin{array}{l}\text { 2. } 96 \\
2.51\end{array}$ & $\begin{array}{r}1.030 \\
\ldots .\end{array}$ \\
\hline & Mean ................. & $50 \frac{1}{8}$ & 10.96 & 89.04 & 63.42 & 11.32 & 2.95 & 1.139 \\
\hline
\end{tabular}

The investigation proves principally that the weight per bushel is hardly a safe guide as to quality, but one must rather judge from the percentage of moisture and nitrogenous constituents which the grain contains. The specimens examined were certainly not extremely starchy, nor were they very dry. Being so few in number, they hardly form a basis for rational conclusion in regard to our grain and its comparison with that of other countries, but they were considered by the editor of the Prag. Agricultural Journal as showing that American barleys were quite equal to those of the Continent. 
The results which have been quoted, while showing that the standard to be reached if possible is a large mealy grain with not more than 8 per cent. of albuminoids as described by Maercker, seem to prove rather conclusively that little barely of this quality is produced on the Contineut or elsewhere. The best ranges in the neighborhood of 9.5 per cent. and from 10 to 11 is a fair average.

The sixty samples from all parts of the United States and twelve from Canada, collected for the present investigation, will, when exam. ined in connection with the previous results at home and abroad, give us a reasonable basis for deciding as to our shortcomings and peculi. arities. .

\section{AMERICAN BARLEY.}

The samples of American barley have been collected through our agents from those parts of the country where it is a crop of prominence. They represent fairly well the production of the United States. The largest number of analyses are not for the largest areas of productionNew York, Wisconsin, and California, which raise more than half the crop-but they are scattered through all the States where any amount of barley is grown. In considering the average features of the crop as it is found in market, regard must be had especially for the figures for Canada and the three States named, although the California barley never reaches our Eastern markets.

The other cereals have been analyzed free from any hulls or chaff. It would have been of interest for comparison to have been able to separate the barleys in the same way. Owing to the close adherence this is very difficult, but in a few cases it was attempted and the analyses of these specimens are given, together with a few of the naked varieties.

The sources of the barleys are described in the following tables. 


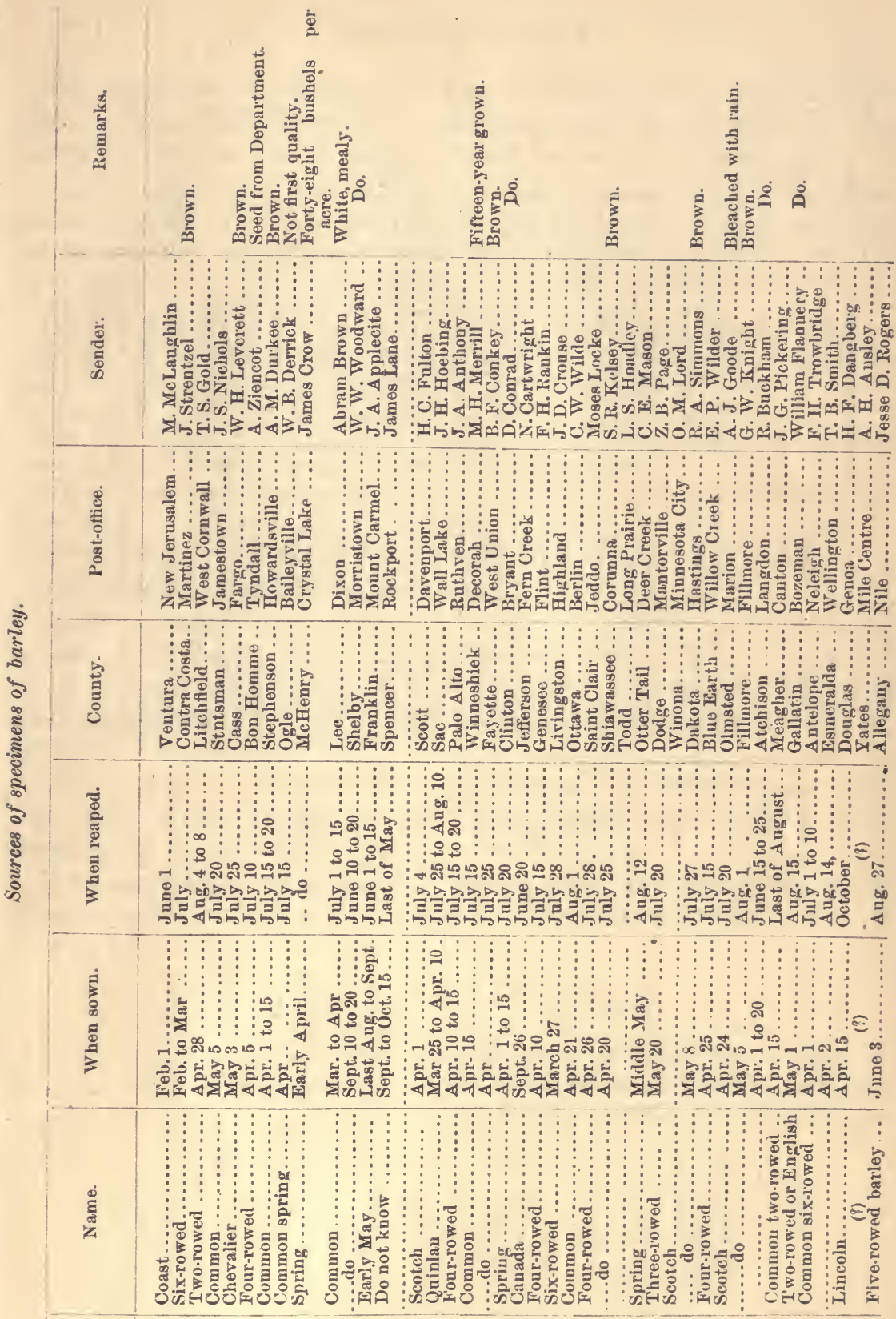

‘دәqumu

\begin{tabular}{|c|c|c|c|c|c|}
\hline 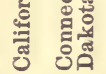 & 总 & 节 & 造 & 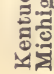 & \\
\hline
\end{tabular}




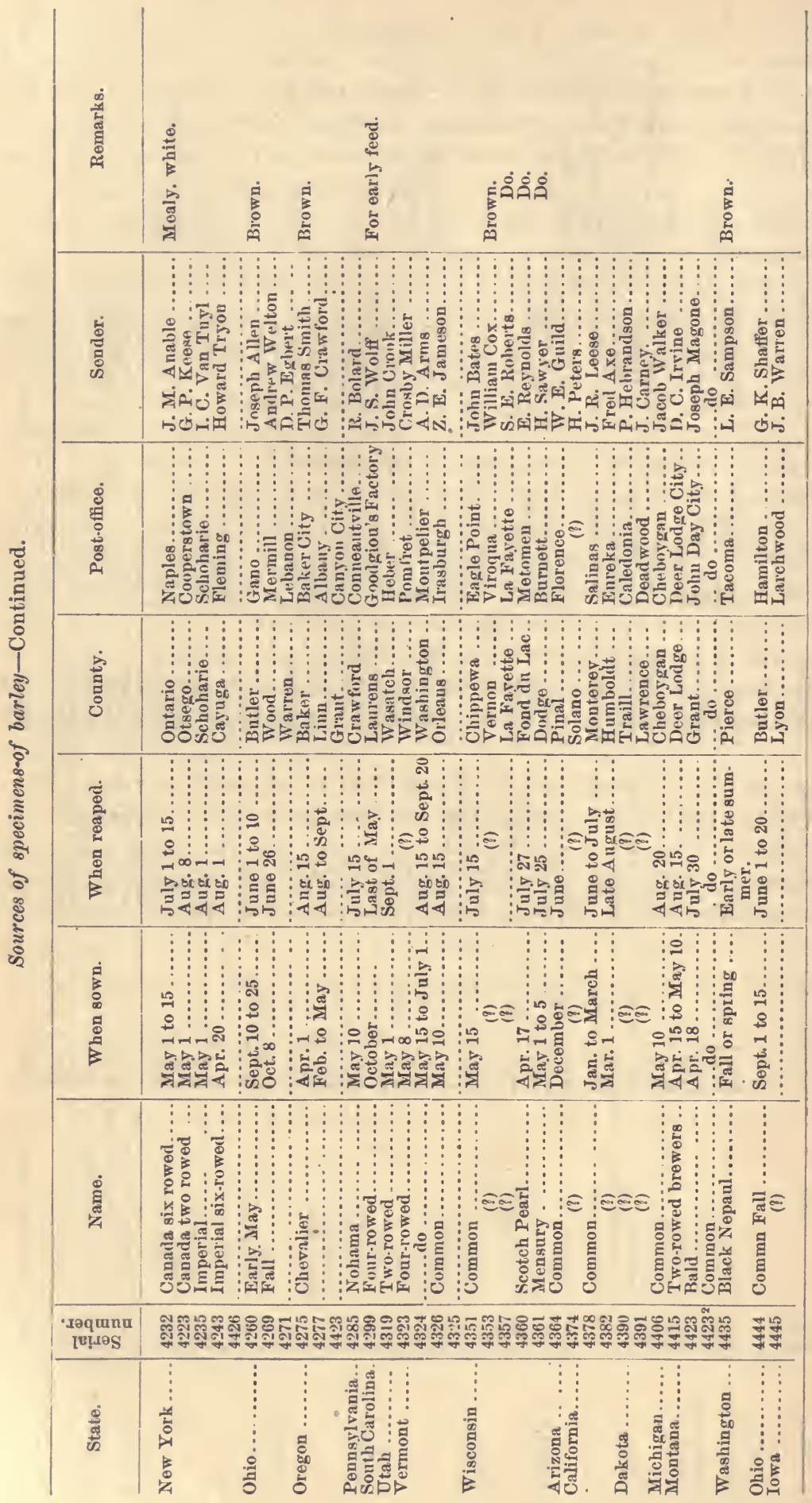




\section{CANADIAN BARLEYS.}

The specimens of Canadian barleys were obtained by application to Hon. A. Blue, of the Bureau of Agriculture and Arts, in Toronto. He forwarded them to the Department with the following letter:

I was not able until yesterday to get the samples of Ontario barley asked for by you for analysis. They were sent on by express, and I trust will reach jon safely. The samples have been collected from four districts of the Province, and graded 1, 2, and 3 by the Government inspector here. The districts are indicated as A, B, C, and $D$, and the localities are shown on the inclosed map.

As a rule, our best barley is produced in the counties north of Lake Ontario, and especially in those bordering on the Bay of Quinte, viz, Prince Edward, Lennox, and Addington and Hastings; but this year it was injured there by rains at the harvest season.

The western district is much more subject to summer rains, owing to its situation between the Great Lakes, and the barley is often discolored.

The brightest grain this year is found in the counties of Peel, York, Ontario, and Durham.

I shall be greatly obliged if jou will send me the results of your analysis.

The districts as indicated included-

A, the counties north of the central part of Lake Erie; B, the counties north of the northwestern part of Lake Ontario; C, the counties north of the central portion of Lake Ontario; D, the counties north of the northeastern portion of Lake Ontario, bordering on the Bay of Quinte.

$\mathrm{B}, \mathrm{C}$, and $\mathrm{D}$ are therefore the best barleys, and especially $\mathrm{D}$, which was, however, unfortunately injured this year, and the brightest grain found in $\mathrm{B}$.

How these practical opinions of quality agree with the facts learned from chemical analysis and with the investigations of Maercker will ap. pear in our discussion of the results.

CHEMICAL AND PHYSICAL DATA.

In the following tables are arranged the data which have been ob. tained from an examination of the specimens which have been described, together with averages for the United States and Canada, and for the various States and geographical divisions: 
Canadian barleys.

\begin{tabular}{|c|c|c|c|c|c|c|c|c|c|c|c|c|c|c|c|}
\hline \multirow{2}{*}{ Grade. } & \multirow[b]{2}{*}{ 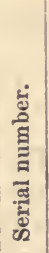 } & \multirow[b]{2}{*}{ 总 } & \multicolumn{7}{|c|}{ Composition. } & \multicolumn{2}{|c|}{ Weight. } & \multicolumn{4}{|c|}{ Consistency. } \\
\hline & & & 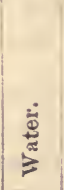 & $\frac{1}{4}$ & ซี่ & 总 & 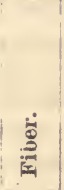 & 递 & 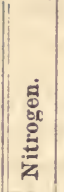 & 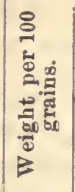 & 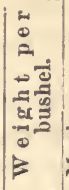 & छై & & 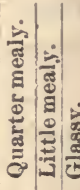 & \\
\hline $\begin{array}{r}\text { First quality } \\
\text { Do } \ldots . . . \\
\text { Do } \ldots . . . \\
\text { Do ..... }\end{array}$ & $\begin{array}{l}6041 \\
6044 \\
6047 \\
6050\end{array}$ & $\mid \begin{array}{l}A^{1} \\
B^{1} \\
C^{1} \\
D^{1}\end{array}$ & \begin{tabular}{|l|} 
P. ct. \\
7.58 \\
8.35 \\
6.95 \\
8.35
\end{tabular} & $\begin{array}{l}\text { P. ct. } \\
2.98 \\
2.73 \\
2.68 \\
2.88\end{array}$ & $\begin{array}{l}P . c t . \\
2.70 \\
2.69 \\
2.64 \\
2.67\end{array}$ & \begin{tabular}{|l|} 
P. ct. \\
73.49 \\
73.23 \\
74.28 \\
73.13
\end{tabular} & $\begin{array}{l}P . c t . \\
\text { 3. } 10 \\
3.55 \\
3.65 \\
3.69\end{array}$ & \begin{tabular}{r|}
$P . c t$ \\
10.15 \\
9.45 \\
9.80 \\
9.28
\end{tabular} & $\begin{array}{l}\text { P.c. } \\
1.62 \\
1.51 \\
1.57 \\
1.48\end{array}$ & $\begin{array}{l}\text { Grm } \\
2.910 \\
3.069 \\
3.206 \\
3.445\end{array}$ & $\begin{array}{l}L b 8 . \\
54.8 \\
56.1 \\
55.9 \\
52.7\end{array}$ & $\begin{array}{l}16 \\
40 \\
12\end{array}$ & $\begin{array}{l}32 \\
48 \\
28 \\
36\end{array}$ & $\begin{array}{l}2420 \\
3612 \\
2012 \\
3616\end{array}$ & \\
\hline A verage & & & 7.81 & 2.82 & $\overrightarrow{2.67}$ & $\overline{73.53}$ & 3.50 & $\overline{9.67}$ & 1.54 & 3.158 & 54.9 & 17 & 36 & 2915 & 3 \\
\hline $\begin{array}{r}\text { Soond quality } \\
\text { Do } \ldots . . . \\
\text { Do } \ldots . . . \\
\text { Do } \ldots . . .\end{array}$ & $\begin{array}{l}6042 \\
6045 \\
6048 \\
6051\end{array}$ & $\begin{array}{l}\mathrm{A}^{2} \\
\mathrm{~B}^{2} \\
\mathrm{C}^{2} \\
\mathrm{D}^{2}\end{array}$ & $\begin{array}{r}7.85 \\
7.03 \\
10.08 \\
8.43\end{array}$ & $\begin{array}{l}2.95 \\
2.80 \\
1.62 \\
3.18\end{array}$ & \begin{tabular}{l|}
2.72 \\
2.80 \\
2.78 \\
2.63
\end{tabular} & \begin{tabular}{|l|}
72.76 \\
73.46 \\
72.58 \\
72.55 \\
\end{tabular} & $\begin{array}{l}3.22 \\
3.76 \\
3.49 \\
3.41\end{array}$ & \begin{tabular}{|r|}
10.50 \\
10.15 \\
9.45 \\
9.80
\end{tabular} & $\begin{array}{l}1.68 \\
1.62 \\
1.51 \\
1.57\end{array}$ & $\begin{array}{l}2.818 \\
3.056 \\
2.934 \\
3.257 \\
\end{array}$ & \begin{tabular}{|l|}
54.5 \\
54.7 \\
53.5 \\
53.5 \\
\end{tabular} & $\begin{array}{l}36 \\
16 \\
12 \\
24\end{array}$ & $\begin{array}{l}40 \\
36 \\
36 \\
40 \\
\end{array}$ & $\begin{array}{ll}12 & 12 \\
28 & 16 \\
32 & 20 \\
32 & 4\end{array}$ & \\
\hline A verage .. & & 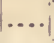 & 8.35 & 2.64 & 2.73 & 72.84 & 3.47 & 9.97 & 1.59 & 3.021 & & 22 & 38 & 2613 & 1 \\
\hline $\begin{array}{r}\text { Third quality ... } \\
\text { Do } \ldots . . . \\
\text { Do } \ldots . . . \\
\text { Do } \ldots . . .\end{array}$ & $\begin{array}{l}6043 \\
6046 \\
6049 \\
6052\end{array}$ & $\begin{array}{l}A^{3} \\
B^{3} \\
C^{3} \\
D^{3}\end{array}$ & $\begin{array}{l}8.78 \\
6.75 \\
8.13 \\
7.93 \\
\end{array}$ & $\begin{array}{l}2.70 \\
2.83 \\
3.05 \\
3.18\end{array}$ & $\begin{array}{l}2.69 \\
2.72 \\
2.67 \\
2.74\end{array}$ & $\begin{array}{l}72.35 \\
73.87 \\
72.82 \\
73.47 \\
\end{array}$ & \begin{tabular}{|l|}
3.50 \\
3.68 \\
3.35 \\
3.35
\end{tabular} & \begin{tabular}{|l|}
9.98 \\
10.15 \\
9.98 \\
9.33
\end{tabular} & $\begin{array}{l}1.60 \\
1.62 \\
1.60 \\
1.49\end{array}$ & $\begin{array}{l}3.012 \\
3.094 \\
2.941 \\
3.226\end{array}$ & $\begin{array}{l}\overline{\overline{52.4}} \\
54.8 \\
52.4 \\
54.3\end{array}$ & $\begin{array}{l}36 \\
16 \\
20\end{array}$ & $\begin{array}{l}28 \\
44 \\
40 \\
32\end{array}$ & \begin{tabular}{ll|}
24 & 8 \\
32 & 8 \\
44 & 16 \\
24 & 20
\end{tabular} & $\begin{array}{l}\overline{4} \\
8\end{array}$ \\
\hline A verage & & & 7.89 & 2.94 & 2.71 & 73.13 & 3.47 & 9.86 & 1.58 & 3.068 & & & 36 & 3111 & 4 \\
\hline $\begin{array}{l}\text { Average A local } \\
\text { Average B local } \\
\text { Average C loca } \\
\text { Average D loca }\end{array}$ & & & \begin{tabular}{|l|}
8.07 \\
7.37 \\
8.39 \\
8.24 \\
\end{tabular} & \begin{tabular}{l|}
2.88 \\
2.79 \\
2.45 \\
3.08 \\
\end{tabular} & $\begin{array}{l}2.70 \\
2.74 \\
2.70 \\
2.68 \\
\end{array}$ & $\begin{array}{r}72.87 \\
73.52 \\
73.23 \\
73.05 \\
\end{array}$ & \begin{tabular}{|l|}
3.27 \\
3.66 \\
3.49 \\
3.48 \\
\end{tabular} & $\begin{array}{l}10.21 \\
9.92 \\
9.74 \\
9.47 \\
\end{array}$ & $\begin{array}{l}1.63 \\
1.58 \\
1.56 \\
1.51 \\
\end{array}$ & $\begin{array}{l}.943 \\
3.073 \\
3.027 \\
3.309 \\
\end{array}$ & $\begin{array}{r}53.9 \\
55.2 \\
53.9 \\
53.5 \\
\end{array}$ & $\begin{array}{l}30 \\
11 \\
19 \\
\end{array}$ & $\begin{array}{l}33 \\
33 \\
36 \\
\end{array}$ & \begin{tabular}{rr|r}
20 & 13 \\
32 & 9 \\
32 & 16 \\
31 & 13 & $\vdots$ \\
\end{tabular} & i \\
\hline Grand average & & & $\overline{8.02}$ & 2.80 & 2.70 & 73.17 & 3.48 & 9.83 & 1.57 & 3.088 & & & 37 & 2913 & 2 \\
\hline
\end{tabular}

Physical properties of barley.

Looality.

Vermout.

Connocticut
Sow York.

Peunsylvania

Ohio.

Michigan

Indiana.

Iliinois

\begin{tabular}{|c|c|c|c|c|c|c|c|}
\hline 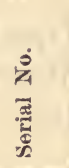 & 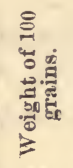 & 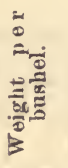 & 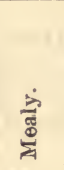 & $\begin{array}{l}\frac{\dot{亠}}{\Xi} \\
\stackrel{\Xi}{\Xi} \\
=\end{array}$ & 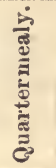 & 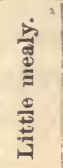 & $\frac{0}{\sigma}$ \\
\hline 4323 & 3. 120 & 52.2 & & 16 & 44 & 24 & 16 \\
\hline 4324 & 3.480 & 51.4 & 40 & 36 & 16 & 8 & \\
\hline 4326 & 2. 980 & 52.4 & ....... & 20 & 40 & 40 & \\
\hline 4028 & 4. 380 & 53. 0 & 12 & 24 & 36 & 28 & \\
\hline 4220 & 3. 390 & 57.7 & ...... & …. & ..... & ....... & \\
\hline 4226 & 2. 880 & 54.5 & ....... & $\ddot{m}$ & & 0 & \\
\hline 4228 & 3. 300 & 51. 4 & $\therefore \ldots$ & 36 & 44 & 20 & \\
\hline 4232 & 3. 570 & 53.4 & 16 & 28 & 28 & 28 & \\
\hline 4233 & 3.380 & 49. 3 & 20 & 40 & 28 & 12 & \\
\hline 4243 I & 3.410 & 54.7 & 8 & 24 & 24 & $44^{\circ}$ & \\
\hline 424311 & 2.690 & 52.9 & & & & & \\
\hline 4285 & 2. 630 & 50.4 & 8 & 36 & 24 & 32 & \\
\hline 4260 & 3. 690 & 53. 9 & 16 & 40 & 32 & 12 & \\
\hline 4269 & 3. 170 & 50.8 & 36 & 36 & 20 & 8 & \\
\hline 4271 & 3. 180 & 52.5 & 40 & 44 & 12 & 4 & \\
\hline 4444 & 2. 980 & 51.0 & 40 & 32 & 20 & 8 & \\
\hline 4151 & 3.450 & 54.3 & 16 & 36 & 40 & 8 & \\
\hline 4153 & 2. 280 & 49. 3 & & 32 & 36 & 24 & 8 \\
\hline 4156 & 3.200 & 51. 3 & 24 & 28 & 28 & 20 & \\
\hline 4158 & 3.260 & 56.8 & 4 & 36 & 44 & 16 & \\
\hline 4160 & 3. 180 & 53.7 & 16 & 40 & 28 & 16 & \\
\hline 4406 & 3. 530 & 58.7 & 24 & 32 & 28 & 16 & \\
\hline 4080 & 3.310 & 53.2 & 24 & 48 & 28 & & \\
\hline 4081 & 3.110 & 53.1 & 12 & 36 & 28 & 24 & \\
\hline 4085 & 3. 570 & 54. 3 & 48 & 22 & 16 & 14 & \\
\hline 4062 & 2.740 & 50.4 & 32 & 28 & 20 & 20 & ...... \\
\hline 4063 & 2.840 & 49.8 & 28 & 32 & 32 & 8 & ....... \\
\hline 4065 & 3. 050 & 52.0 & 24 & 36 & 24 & 16 & - \\
\hline 4067 & 2.920 & 52.2 & 12 & 28 & 24 & 36 & \\
\hline
\end{tabular}


Physical properties of barleys-Continued.

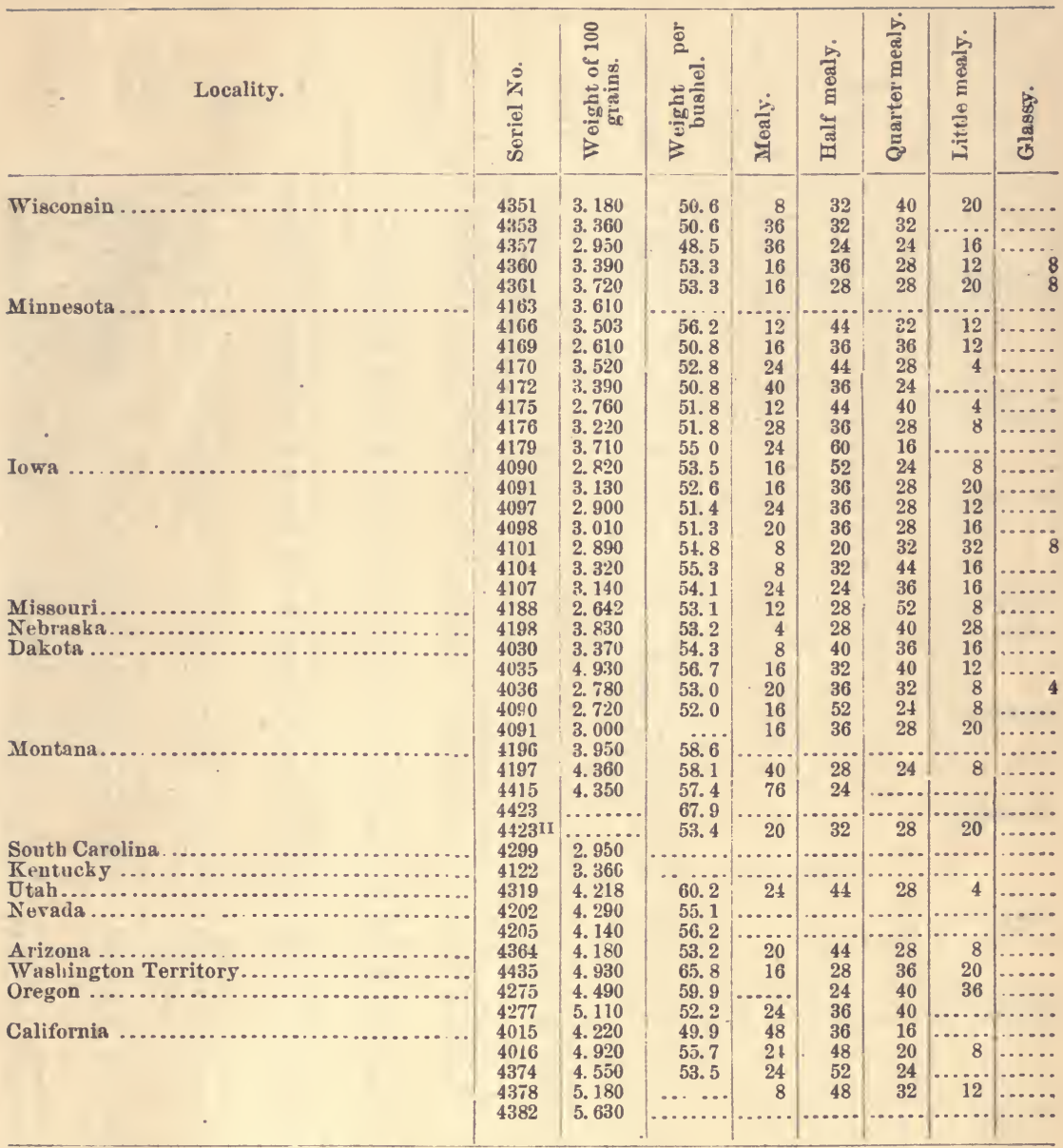

Average physical properties of American barleys.

\begin{tabular}{|c|c|c|c|c|c|c|c|c|}
\hline . & $\begin{array}{l}\text { No. of } \\
\text { determi- } \\
\text { nations. }\end{array}$ & $\begin{array}{l}\text { Weight per } \\
100 \text { graius. }\end{array}$ & $\begin{array}{c}\text { Weight } \\
\text { per bushel. }\end{array}$ & Mealy. & $\begin{array}{c}\text { Half } \\
\text { mealy. }\end{array}$ & $\begin{array}{l}\text { Quarter } \\
\text { mealy. }\end{array}$ & $\begin{array}{l}\text { Little } \\
\text { mealy. }\end{array}$ & Glassy. \\
\hline 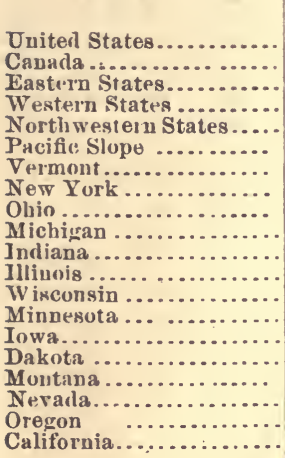 & \begin{tabular}{r|}
76 \\
12 \\
13 \\
39 \\
10 \\
12 \\
3 \\
8 \\
4 \\
4 \\
6 \\
3 \\
5 \\
5 \\
7 \\
7 \\
5 \\
5 \\
2 \\
2 \\
5
\end{tabular} & $\begin{aligned} \text { Grams. } \\
3.482 \\
3.088 \\
3.016 \\
3.171 \\
3.680 \\
4.655 \\
3.193 \\
3.217 \\
3.230 \\
3.150 \\
3.330 \\
2.890 \\
3.320 \\
3.290 \\
3.030 \\
3.354 \\
4.220 \\
4.215 \\
4.800 \\
4.900\end{aligned}$ & \begin{tabular}{r|} 
Pounds. \\
54.0 \\
54.1 \\
52.6 \\
52.8 \\
57.2 \\
56.8 \\
52.0 \\
53.1 \\
52.1 \\
54.0 \\
53.5 \\
51.1 \\
51.3 \\
52.7 \\
53.4 \\
54.2 \\
59.1 \\
55.7 \\
56.1 \\
53.0
\end{tabular} & $\begin{array}{l}20 \\
19 \\
11 \\
21 \\
27 \\
\text { i1 } \\
13 \\
11 \\
33 \\
14 \\
28 \\
28 \\
22 \\
22 \\
17 \\
15 \\
45 \\
13 \\
12 \\
26\end{array}$ & $\begin{array}{l}35 \\
37 \\
29 \\
35 \\
35 \\
40 \\
24 \\
32 \\
38 \\
34 \\
35 \\
32 \\
30 \\
43 \\
34 \\
39 \\
28 \\
30 \\
30 \\
46\end{array}$ & $\begin{array}{l}29 \\
29 \\
32 \\
30 \\
26 \\
29 \\
34 \\
31 \\
21 \\
34 \\
24 \\
25 \\
31 \\
29 \\
32 \\
32 \\
17 \\
37 . \\
40 \\
23\end{array}$ & $\begin{array}{r}15 \\
13 \\
26 \\
13 \\
11 \\
10 \\
24 \\
26 \\
8 \\
16 \\
13 \\
15 \\
14 \\
6 \\
16 \\
13 \\
10 \\
73 \\
18 \\
5\end{array}$ & $\begin{array}{l}0 \\
1 \\
0 \\
0 \\
3 \\
0 \\
1 \\
1 \\
0 \\
0 \\
0 \\
0\end{array}$ \\
\hline
\end{tabular}


Composition of American barleys (unhulled), arranged by States.

\begin{tabular}{|c|c|c|c|c|c|c|c|c|}
\hline State. & 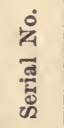 & 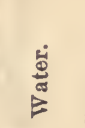 & $\frac{5}{4}$ & $\overline{\tilde{\sigma}}$ & 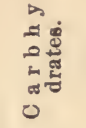 & $\frac{\grave{\$}}{\grave{m}}$ & 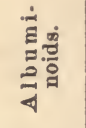 & 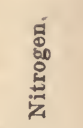 \\
\hline & & er ct. & Per et. & Per ct. & Per ct. & Per ct. & Per ct. & $r e t$ \\
\hline \multirow[t]{3}{*}{ 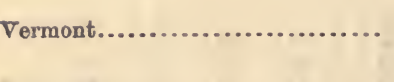 } & 4323 & $6-70$ & 2.22 & 2. 90 & 70.28 & 3. 90 & & 2.24 \\
\hline & 4324 & 6. & & 2. & 72. 37 & 8 & 12. & 2.02 \\
\hline & $43: 6$ & 6.55 & 2.90 & 2.75 & 71.57 & 4. 15 & 12.08 & 1.93 \\
\hline Connecticut & 4028 & 6.50 & 2.99 & 2.33 & 75.14 & 2.89 & 10.15 & 1.62 \\
\hline \multirow{2}{*}{ 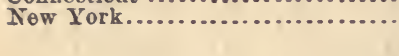 } & 4226 & 6.86 & 2.40 & 2.76 & 72.85 & 3.40 & 11.73 & 1.88 \\
\hline & $4 \% 28$ & 6.77 & 2.12 & 2. 77 & 73. 41 & 3.55 & 11.38 & 1.82 \\
\hline & 4232 & 5. 90 & 2.70 & 2.58 & 74.14 & 3.05 & 11.03 & 1.76 \\
\hline & 4233 & 6.95 & 2.64 & 2.66 & 74.47 & 3. 13 & 10.15 & 1.62 \\
\hline \multirow{3}{*}{ 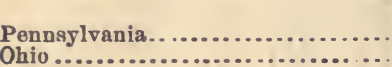 } & 4243 & 7.39 & 2.45 & 2.48 & 73. 10 & 3. 73 & 10.85 & 1.74 \\
\hline & 4275 & 6.27 & 3. 05 & 2.06 & $72 \cdot 89$ & 3. 83 & 11. 90 & 1.90 \\
\hline & $4: 60$ & 6.85 & 3. 311 & 3.53 & 71.84 & 3. 80 & 10.68 & 1.71 \\
\hline \multirow{3}{*}{ (n) } & 4269 & 6.2 .5 & 3. 07 & 2.40 & 73. 13 & 4. 65 & 10. & 1.68 \\
\hline & 4271 & 6.81 & 3.55 & 2.58 & 72.91 & 4. 00 & 10.15 & 1.62 \\
\hline & 4444 & 6.80 & 3.10 & 2. 06 & 73.92 & 4.32 & 9.80 & 1. 57 \\
\hline \multirow{3}{*}{ Michigan...$\ldots \ldots \ldots \ldots \ldots \ldots \ldots \ldots$} & 4151 & 6.44 & 2.97 & 2.70 & 71.33 & 3.43 & 13.13 & 2.10 \\
\hline & 4153 & 6.37 & 2. 59 & 2. 73 & 69. 73 & 388 & 14. 70 & 2. 35 \\
\hline & 4158 & 6.73 & 2.56 & 2.90 & 71.83 & 3.03 & 12 & 2.07 \\
\hline & 4160 & 5. 27 & 3. 05 & 2.71 & 73.36 & 3.71 & 11.90 & 1.90 \\
\hline & 4406 & 6.55 & 2.75 & 2.55 & 75.45 & 3.07 & 9.63 & 1.54 \\
\hline Indiana... & 4080 & 5. 99 & 3.50 & 3.54 & 71.19 & 4.40 & 11.38 & 1.82 \\
\hline & $40 \times 5$ & 5.92 & 2.95 & 2.73 & 75. & 3.58 & 9.45 & 1.51 \\
\hline \multirow{3}{*}{ 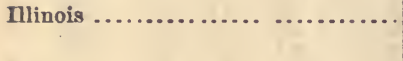 } & 4062 & 6. 06 & 3. 34 & 2.61 & 70. & 4.51 & 12.60 & 2.02 \\
\hline & 4063 & 6.18 & 3. 16 & 2. 59 & 72.55 & 4.14 & 11. & 1.82 \\
\hline & 4065 & 6. 72 & 2.73 & 2.81 & 72. & 2. 64 & 12.95 & 2.07 \\
\hline (1) & 41167 & 6.52 & 3.08 & 2. 66 & 71.77 & 3. 37 & 12.60 & 2.02 \\
\hline \multirow{3}{*}{ Wisconsin..............} & 4351 & 7.15 & 2.90 & 2. 76 & 70.51 & 4. & 12.25 & 1. 96 \\
\hline & 4353 & 7.40 & 2. 30 & 2.74 & 73. 17 & 3 & 11.50 & 1. 68 \\
\hline & 4357 & 6.60 & 3. 60 & 2. & 72. & 4.27 & 10. & 1. 74 \\
\hline \multirow{2}{*}{ 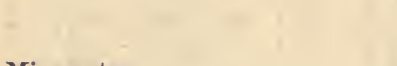 } & 4360 & 7.70 & 2. 75 & 2.50 & 72.77 & 3. 78 & 10 & 1. 68 \\
\hline & 4361 & 6.40 & 3. 15 & 2. 49 & 70.88 & 3.95 & 13. & 2.10 \\
\hline \multirow{5}{*}{ 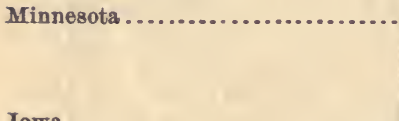 } & 4169 & 7.60 & 1. 50 & 2. 69 & 73. 79 & 3.57 & 10.85 & 1. 74 \\
\hline & 4170 & 6. & 3. 00 & 3. & 73. & 4.40 & 9. & 1.51 \\
\hline & 4172 & 6.30 & 2.51 & 2. 76 & 74.72 & 4.43 & 9.28 & 1.4 \\
\hline & 4175 & 7. & 3.15 & 2. 80 & 71. & 3. & 11.90 & 1.90 \\
\hline & $4176^{\circ}$ & 9.15 & 2. 97 & 2. 72 & 70.69 & 3. 97 & 10.50 & 1.6 \\
\hline \multirow{5}{*}{ Iowa $\ldots \ldots \ldots \ldots \ldots \ldots \ldots \ldots \ldots \ldots$} & 4091 & 6.47 & 2. 85 & 2. 63 & 71. & 3. & 12. & 2.04 \\
\hline & 4098 & 5. 69 & 3. $1 \times$ & 2. 75 & 72.28 & 4. 37 & 11.73 & 1. \\
\hline & 41111 & 6. 24 & 2. & 2. 83 & 72. & & & 1. \\
\hline & 4104 & 6.67 & 3. 33 & 2.65 & 69. 19 & 3. 81 & 14. & 2.3 \\
\hline & 4198 & 7.58 & 3. & 2. 70 & 71.12 & 3. 35 & & 1. \\
\hline \multirow{5}{*}{ Dakota } & 40311 & 5. 80 & 3. & 3. 01 & 69. 97 & 3. 29 & 14. & 2. 38 \\
\hline & 4035 & 5. 55 & 2. & & & & & 1.85 \\
\hline & 4036 & 5.7 & 3. & 2.94 & 71. & 3. & 13. & 2. \\
\hline & 4390 & & 3. & & & & & 1. \\
\hline & 4391 & 5.95 & 2.65 & 2.68 & 71.34 & 4. 25 & 13. & 2.10 \\
\hline \multirow{3}{*}{ 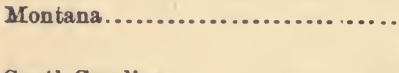 } & 4196 & 7.55 & 1.70 & 2. & 74. 53 & & & 1.54 \\
\hline & 4197 & 6. & 3. 00 & 2.52 & 74.20 & 3. & 10. & 1. \\
\hline & 4415 & 4. & 3.1 & & 76. & 3. & & 1. 51 \\
\hline South Carolina... & $4 \div 99$ & 6. 8 & 2. & 2. & 73. & 4. 10 & 10. & 1.65 \\
\hline & 4122 & 6. & 2. & & & & 8. & 1.40 \\
\hline - & 4319 & 7. & 3.4 & 2.53 & 72.99 & 2. 88 & 10.50 & 1. 68 \\
\hline & 4364 & 6.: & 2.9 & 2. & 74.30 & & 9.63 & 1.54 \\
\hline 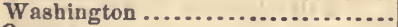 & $44: 35$ & 5.95 & 3. 50 & 2.98 & 70.97 & 4. 35 & 12.25 & 1.96 \\
\hline Oregon ............... & 4275 & 6.27 & 3. 0.5 & 2.06 & 72. & 3.83 & 11.90 & 1. 90 \\
\hline & $427 i$ & 6.20 & 2.78 & 2.71 & 75.56 & 4. 00 & 8.75 & 1.40 \\
\hline California... & 4016 & 6.70 & 2. 74 & 3.01 & 74. & 4. & 9.10 & 1.46 \\
\hline & $4: 374$ & 4.53 & 4.43 & 2. 72 & 74. & 4.48 & 9.10 & 1.46 \\
\hline & $437 \times$ & 6. 18 & 2.74 & 2.50 & 75. & 4. & 8.93 & 1.43 \\
\hline ng. & 4423 & 6.70 & 2.20 & 52 & 74.03 & 3.00 & 11.55 & 1. 85 \\
\hline Colorado ........ & 3584 & 8.15 & 2.77 & 2.87 & 68.99 & 3.92 & 13. 30 & 2.13 \\
\hline
\end{tabular}


Average composition of American barleys (unhulled), arranged by States.

\begin{tabular}{|c|c|c|c|c|c|c|c|c|}
\hline State. & 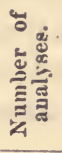 & 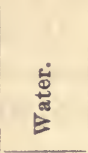 & $\frac{\pi}{4}$ & $\overline{0}$ & 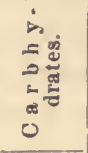 & $\frac{\overrightarrow{0}}{4}$ & 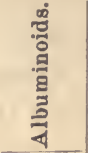 & 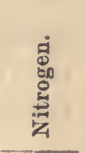 \\
\hline United States. & 60 & $\begin{array}{r}\text { Per ct. } \\
6.53\end{array}$ & $\begin{array}{r}\text { Per ct. } \\
2.89\end{array}$ & $\begin{array}{l}\text { Per ct. } \\
2.6 \times\end{array}$ & $\begin{array}{r}\text { Per } c t . \\
72.77\end{array}$ & $\begin{array}{r}\text { Per ct. } \\
3.80\end{array}$ & $\begin{array}{c}\text { Per ct. } \\
11.33\end{array}$ & $\begin{array}{r}\text { Per ct. } \\
1.81\end{array}$ \\
\hline Atlantic Slope... & 10 & 6. 64 & 2.51 & 2. 59 & 73.02 & 3.57 & 11. .39 & 1.85 \\
\hline Northern States. & 48 & 6. 55 & 2. 87 & 2.69 & 72.53 & 3. & $11.5 x$ & 83 \\
\hline Western States ... & 30 & 666 & 2. 96 & 2.73 & 72. & 3. & 11.52 & 1. 84 \\
\hline Northwestern States............... & 8 & 6. 02 & 2.85 & 2. 69 & 73. & 3. 59 & 11.82 & \\
\hline 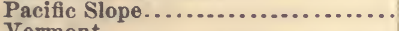 & 10 & 6.47 & 3.05 & 2.65 & 72.43 & 3. & 30 & 1. 69 \\
\hline Vermont ............. & 3 & 6. $5 \delta$ & 2.51 & 2.77 & $71.4 \mathrm{l}$ & 3.84 & 12.89 & 2. 06 \\
\hline 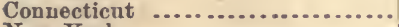 & 1 & 6.50 & $\because 99$ & 2.33 & 75. & 2. & 10.15 & 1.62 \\
\hline New York.... & 5 & 6. 77 & 2.46 & 2.65 & 73. 59 & 3. 50 & 11.113 & 1. 76 \\
\hline Pennsylvania......................... & 1 & 6. 27 & 3. 05 & 2. 06 & 7:. & 3 & 90 & 1. \\
\hline 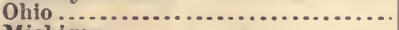 & 4 & 6. 68 & 3.25 & 2.64 & 72.95 & 4. 1 & 10.26 & 1. \\
\hline Michigan...$\ldots \ldots \ldots \ldots \ldots \ldots \ldots \ldots$ & 5 & 13. 27 & 2.79 & 2. 72 & 72. & 3. & 12.46 & 1. \\
\hline Indiana...$\ldots \ldots \ldots \ldots \ldots \ldots \ldots \ldots \ldots$ & 2 & 5.9 .5 & 3.23 & 3. 13 & 73.28 & 3.99 & 10.42 & 1.67 \\
\hline Illinois $\ldots \ldots \ldots \ldots \ldots \ldots \ldots \ldots \ldots$ & 4 & 6. 37 & 3. & 2.67 & 71.84 & 3. & 12.38 & 1. \\
\hline Wisconsin...$\ldots \ldots \ldots \ldots \ldots \ldots \ldots \ldots \ldots$ & 5 & 7.05 & 2.95 & 2. 63 & 71.87 & 4. & 11.45 & 1. \\
\hline Minnesota................ & 5 & 7.29 & 2. & 2. & 72.87 & & $30 \quad 39$ & 1.66 \\
\hline 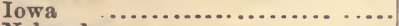 & 4 & 6. 27 & 3.08 & 2.71 & 71.37 & 4. & 12.56 & 2.01 \\
\hline Nebraska............ & 1 & 7. 5 & 3. & 2.70 & 71. & & 12. 2.5 & 1.96 \\
\hline Dakota ................................. & 5 & 5.8 & 2. 99 & 2.77 & 71.7 & 3. & 13. 02 & 2. \\
\hline Montana................ & 3 & 6. 3 & 2.62 & 2.56 & 75. 1 & 3.48 & 9. 80 & 1. 57 \\
\hline 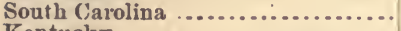 & 1 & 6. 8 & $2.6 j$ & $2.4 . j$ & 73. 62 & 4. & 10.33 & 1.65 \\
\hline Kentucky ...................... . & 1 & 6. 0 & 2.90 & 2.37 & 7... 73 & 4. 25 & 8.75 & 1.40 \\
\hline ................ & 1 & 7.70 & 3.40 & 2.33 & 72.99 & 2.88 & 10. 50 & 1. 68 \\
\hline A rizona & 1 & 6.2 & 2. & 2. & 74.30 & 4. 28 & 9. 63 & 1.54 \\
\hline Washington ............ & 1 & 5.9 & 3.50 & 2.98 & 70.97 & 4. 35 & 12. 25 & 1.96 \\
\hline & 2 & 6. 23 & 2.92 & 2.38 & 74. $2: 3$ & 3. 91 & 10. 33 . & 1.65 \\
\hline Califiorn & 3 & 5.80 & 3. 30 & 2.74 & 74. 86 & 4.25 & 9. 115 & 1.45 \\
\hline Wyoming...$\ldots \ldots \ldots \ldots \ldots$ & 1 & 6. 70 & 2. 20 & 2.52 & 74. $0: 3$ & 3.00 & 11.5 .5 & 1.85 \\
\hline Colorado.......................... & 1 & 8. 15 & 2. 77 & 287 & 68.99 & 3. 92 & 13. $3 v$ & 2. 13 \\
\hline
\end{tabular}

DISCUSSION OF THE DATA AND AVERAGES.

As Canadian barley forms the greater portion of our supply, it will be considered first, and that of the United States compared with it.

Maercker found that the finest grain contained not more than 8 per cent. of albuminoids and consisted of at least 80 per cent. of mealy kernels. These two factors, together with the brightness of the grain, he considered to be the characteristics by which its quality should be judged.

Of the twelve typical specimens of last year's Canadian crop noue were below 9 per cent. of albuminoids, the average being 9.83 , and only six contained 60 per cent. of keruels which were mealy or half mealy in structure. They cannot be said therefore to be equal to what are considered extremely fine barley in Germany. They do, howerer, reach and in most cases exceed the average production of foreign countries, and may be considered as of extremely good quality for samples from actual trade lots, and better than those produced the world over, as may be seen by comparison with the investigations which have been quoted on previous pages. In weight per bushel they are about the same as the average of Maercker, and in moisture, as with all our grain, much drier than the product of damper climates.

The differences in the different grades are marked almost entirely by brightness and perfection of the kernel, there being a remarkably clos 
agreement in all other respects. This shows how important a factor climate and care in harresting and handling are in enhancing or depreciating the value of the grain. The latter factor, care, is almost entirely within the control of the farmer, while varying seasons, of course, influence the former. Of the different districts that north of Lake Erie produces the specimens richest in nitrogen, which would therefore be graded lowest as far as this influences our judgment, thus agreeing with current opinion. In mealiness these specimens are much ahead of all the others, and this ought to more than balance the slightly higher percentage of albuminoids. The summer rains, however, by coloring the grain have the greatest influence in determining quality, and eventually make the barley of this clistrict the least desirable. The remaining districts, north of Ontario, produce grain much alike, that from the $\mathrm{B}$ district averaging heavier in weight per bushel, and that from $\mathrm{D}$ being a little less nitrogenous. As a whole these Canadian barleys certainly form a very good standard of reference.

\section{BARLEYS OF THE UNITED STATES.}

In comparing the barleys of the United States with those of Canada, it appears at once that, as a whole, the former average about as mealy in consistency as the latter. Examined by distribution geographically, the Eastern grain is found to be much less mealy than the Western, that of the Northwest being the richest in mealy kernels. Again, howerer, we find that but two out of sixty-four samples contained 80 per cent. or over of mealy or half mealy kernels. In weight per bushel there is no variation from Canadian and foreign grain, but in size the barleys of the United States, as a whole, are larger than those of Canada. Unfortunately we have no data for those of foreign production. The Eastern grain is no larger than the Canadian, and the average is increased by the large size of that from the Northwest and the Pacific Slope. which at the same time has an increased weight per bushel.

In brightness, the samples from those portions of the country having a dry climate at harvest time, especially the Pacific Slope and the Northwest, were far superior. This is an important feature in considering the best areas for the production of good malting barley; and while California as yet furnishes almost nothing for brewing purposes, it would seem to be one of our best fields. The high percentage of albuminoids stored up in the peculiar climate of the Northwest, while an advantage in the wheat grain, would be a serious objection in barley. In this respect it appears that the average amount of albuminoids in the barley of the United States is greater than that of Canada, and far ahead of anything which Maercker would consider desirable. California alone is 1 per cent. below the average for the rest of the country, there being 
less than one-half per cent. difference from 11.50 per cent. in the average for all but California, which has 10.50 per cent. This is higher than was found in the Canadian grain, so that it may safely be said that the latter is at present the best in the market and superior to our own.

Among the analyses the following extremes are found:

\begin{tabular}{|c|c|c|c|c|}
\hline f & Highest. & State. & Lowest. & State. \\
\hline 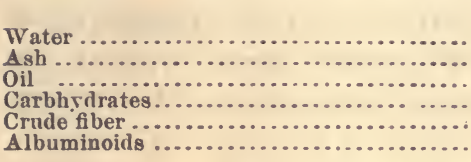 & $\begin{array}{r}\text { Per cent. } \\
9.15 \\
4.43 \\
3.54 \\
76.79 \\
4.65 \\
14.88\end{array}$ & $\begin{array}{l}\text { Minnesota.. } \\
\text { California.. } \\
\text { Indiana..... } \\
\text { Montana ... } \\
\text { Ohio ........ } \\
\text { Dakota..... }\end{array}$ & $\begin{array}{r}\text { Per cent. } \\
\text { 4. } 53 \\
1.50 \\
2.06 \\
68.99 \\
2.64 \\
8.75\end{array}$ & $\begin{array}{l}\text { California. } \\
\text { Minnesota. } \\
\text { Orugou. } \\
\text { Colurado. } \\
\text { Illinois. } \\
\text { Kentueky and Oregon. }\end{array}$ \\
\hline $\begin{array}{l}\text { Weight of } 100 \text { grains } . . . \ldots \ldots \ldots \ldots \text {. drams... } \\
\text { Weight per bushel .............. pounds.. } \\
\text { Per et. of mealy and half nealy kernels .... }\end{array}$ & $\begin{array}{l}4.900 \\
60.2 \\
100.00\end{array}$ & $\begin{array}{l}\text { California.. } \\
\text { Utah ........ } \\
\text { Montana.... }\end{array}$ & $\begin{array}{l}2.630 \\
50.4 \\
16.0\end{array}$ & $\begin{array}{l}\text { Pennsylvania. } \\
\text { Do. } \\
\text { Vermont. }\end{array}$ \\
\hline
\end{tabular}

Dakota sustains its reputation for high nitrogen and Oregon for low, but the variations in this constituent are not as wide as in wheat, barley, like rye, appearing to be less affected in this respect, although Maercker's experiments show that barley responds in its percentage of albuminoids readity to nitrogenous manuring. His seed was, however, very poor in albuminoids-7.7-8.0 per cent.-and would naturally increase when the conditions were made farorable.

In Koenig's collection of analyses of this grain he gives as the average of 127 specimens:

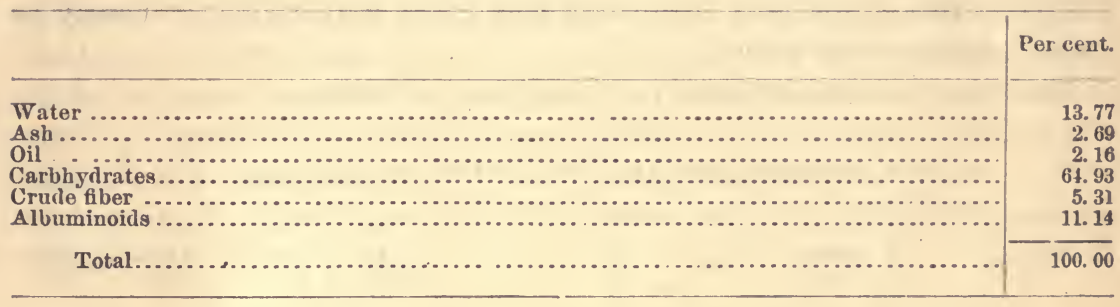

This is bnt little different from the average production of the United States, and would point to the fact that our country, at any rate in certain portions, produces as good malting barley as others. Canada is a witness to this fact, as shown by the specimens which have been examined from there, which are well above foreign averages in starchiness. Experience and care have taught the Canadians, in connection with their favorable climate, the means of producing an excellent grain, superior to other parts of the country. It seems quite possible for the farmers in many portions of the United States, and especially California, the climatic conditions of which are such as to aroid damaging summer rains, with no too dry and hot a climate, to increase our supply of barley of good quality by attention to the conditions which 
have been mentioned, and thus prevent the necessity of importing grain which should be produced at home.

There is one condition which in the case of wheat was found to be of erident effect. Although almost all the specimens examined were spring-sown grain, twelve of winter barley were found to contain but 10.05 per cent. of albuminoid, as compared to 11.42 in the spring varieties. Whether this could be made of any importance in practice cannot of course be decided except by the possibilities of the culture of winter barleys, which as ret seem to be small. Our dry and hot climate, ripen. ing the grain before it has had time to fill out the kernel with starch, and the liability to discoloration from summer showers, are the two dis. advantages we have to contend with.

In a few samples the hull or husk was detached from the grain and the amount determinerl.

Barley-percentage of grain and hulls.

\begin{tabular}{|c|c|c|c|c|c|}
\hline Number. & Grain. & Hull. & Number. & Grain. & Hull. \\
\hline $\begin{array}{l}4015 \\
4081 \ldots \ldots \ldots \\
4090 \ldots \ldots \\
4097 \ldots \ldots \ldots \\
4107\end{array}$ & $\begin{array}{r}\text { Per cent. } \\
83.06 \\
83.78 \\
83.70 \\
84.25 \\
85.72\end{array}$ & $\begin{aligned} & \text { Per cent. } \text { 16. } 94 \\
& 16.22 \\
& 16.30 \\
& 15.75 \\
& 14.28\end{aligned}$ & $\begin{array}{l}4179 \ldots \ldots \ldots \ldots \ldots \\
4202 \ldots \ldots \ldots \ldots \ldots \\
4205 \ldots \ldots \ldots \ldots \ldots \\
4220 \ldots \ldots \ldots \ldots \ldots\end{array}$ & $\begin{array}{r}\text { Per cent. } \\
86.28 \\
84.93 \\
87.45 \\
84.96\end{array}$ & $\begin{array}{r}\text { Per cent. } \\
13.72 \\
15.07 \\
12.55 \\
15.04 \\
\end{array}$ \\
\hline 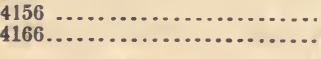 & $\begin{array}{l}84.01 \\
84.47\end{array}$ & $\begin{array}{l}15.99 \\
15.53\end{array}$ & A verage..... & 84.78 & 15.22 \\
\hline
\end{tabular}

The extreme amounts are 16.94 and 12.55 per cent; not nearly as large as is the case with oats.

The composition of these specimens was as follows:

Composition of American barleys (hulled), arranged by States.

\begin{tabular}{|c|c|c|c|c|c|c|c|c|}
\hline State. & $\begin{array}{c}\text { Serial } \\
\text { number. }\end{array}$ & Water. & A 8 h. & Oil. & $\begin{array}{c}\text { Carb- } \\
\text { hydrates. }\end{array}$ & Fiber. & $\begin{array}{c}\text { Albu. } \\
\text { minoids. }\end{array}$ & $\begin{array}{l}\text { Nitro- } \\
\text { gen. }\end{array}$ \\
\hline & & Per cent. & Percent. & Per cent. & Per cent. & Per cent. & Percent. & Per cent. \\
\hline New York......... & 4220 & 6.88 & 1.88 & 2.20 & 75.22 & 1.22 & 12.60 & 2.02 \\
\hline Mo.............. & 4285 & 6.25 & 2.40 & 2. 60 & 76. 27 & 1.98 & 10.50 & 1.68 \\
\hline (n......... & 4156 & 5.55 & 2. 35 & 2.84 & 76. 14 & 1.74 & 11.38 & 1.82 \\
\hline Indiawa ............. & 4081 & 6.55 & 2. 2 & 2. & 7.3. 7 & 1.8 & 13. 30 & 2.13 \\
\hline Minnesota......... & 4166 & 5. 6 & 2. & 2. & 76.91 & 1. & 11.55 & 1.85 \\
\hline Do.............. & 4179 & 6.00 & 2.10 & 2.76 & 76. 35 & 1.41 & 11.38 & 1.82 \\
\hline Iowa... & 4090 & 6.41 & 2.15 & 3. 12 & 74.67 & 2.27 & 11.38 & 1.82 \\
\hline Do... & 4097 & 6.35 & 1. 98 & 2.65 & 75.52 & 1.25 & 12.25 & 1. 96 \\
\hline Do. & 4107 & 6.2 & 2. & & 7.5. 19 & 1.40 & 12.25 & 1. 96 \\
\hline Missunti........... & 4188 & 7.50 & 2. 112 & 2.81 & 73.95 & 1.47 & 12.25 & 1. 96 \\
\hline Nevada.......... & 4202 & 7.2 & 2.3 & & 75.93 & 1.92 & 9.80 & 1.57 \\
\hline |111)................. & 4205 & 2.87 & 1.99 & 2.47 & 81.31 & 1.73 & 9.63 & 1.54 \\
\hline California.......... & 4015 & 5.8 & 2.60 & 2.61 & 76. 03 & 1.23 & 11.73 & 1.88 \\
\hline Do ............... & 4382 & 6.8 & 2.05 & 2.61 & 74.49 & 1.40 & 12. 60 & 2. 02 \\
\hline Colorado........... & 3584 & 7.7 & 2. 30 & 2.86 & 71.10 & 1.90 & 14.00 & 2. 24 \\
\hline
\end{tabular}


Average composition of American barleys (hulled), arranged by States.

\begin{tabular}{|c|c|c|c|c|c|c|c|c|}
\hline State. & 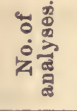 & Water. & Ash. & Oil. & $\begin{array}{c}\text { Carb- } \\
\text { hydrates. }\end{array}$ & Fiber. & $\begin{array}{c}\text { Albumi- } \\
\text { noids. }\end{array}$ & $\begin{array}{c}\text { Nitro. } \\
\text { gen. }\end{array}$ \\
\hline United & & Per cent. & Per cent. & Per cent. & Per cent. & Per cent. & Per cent. & \\
\hline Norther & 10 & 6. 34 & 2. 14 & $\begin{array}{l}2.00 \\
2.66\end{array}$ & 75.40 & 1. 58 & 11. 88 & 1.90 \\
\hline Western Sta & 5 & 6.10 & 2. 27 & 2. & 75.78 & 1. & 11.55 & 1. \\
\hline New York. & 2 & 6. 5 & 2. & 2. & 75.74 & 1. & 11.55 & 1. 85 \\
\hline Michigan & 1 & 5. & 2. & 2. & 76. & 1. & 11 & 1. \\
\hline Indiana. & 1 & 6. 5 & 2. & 2.30 & 7377 & 1.88 & 13. 30 & 2. 13 \\
\hline Minnesota & 2 & อ. & 2. & 2. & 76. & 1. & 11. & \\
\hline Iowa....... & 3 & 6.3 & 2. 0 & 2. 84 & 75. 13 & 1. 64 & 11.96 & 1. \\
\hline Missonri & 1 & 7. & 2. & & 73. & 1. & 12. & 1. \\
\hline Nerada & 2 & 5.0 & 2.2 & 2.62 & 78.62 & 1.8 & 9.71 & 1.5 \\
\hline California ........ & 2 & 6. & 2. & & 75. & $1 .:$ & 12.16 & 1.9 \\
\hline Colorado.... & 1 & 7. 78 & 2.30 & 2.86 & 71.16 & 1.90 & 14.00 & 2. 2 \\
\hline
\end{tabular}

The changes are merely such as one would expect from the remoral of the fibrous hull. The percentages of albuminoids, fiber, and carbhydrates are increased, that of ash and water diminished. The results are merely of value to serve as a comparison of this cereal in its hull-less condition with the other cereals in a similar state.

Our investigations as a whole seem to prove that, while at present Canadian barleys are superior to those grown in the United States, the result is due more to a lack of understanding of the proper localities and methods of cultivation than in any obstacle in the way of extending the production to an extent to do away with our dependence on importation. Field experiments are now most desirable as a means of deciding upon the best varieries and methods as soon as a study of the climatic conditions shall euable us to select those portions of the conntry best suited to this cereal. In time, no doubt, California, whose climate in mans parts is well adapted to the growth of barley for malting pur. poses, will make itself felt if, as appears probable, the quality of ber barleys in the market answers to the expectations raised by laboratory examination.

ANALYSES OF OATS, BARLEY, AND RYE IN DE'TAIL.

In our first report several analyses of wheat were published in which the carbhydrates were separated into their proportious of sugars, starch, and gum, and the albuminoids into those soluble in alcohol of 80 per cent. strength and those insoluble. In the Annual Report of the Department for 1878 several analyses of corn were presented in the same way. For comparison with these results, which are of interest, several have been made of oats, barley, and rye: 
Annlyses of oats, barley, and rye in detail.

OATS.

\begin{tabular}{|c|c|c|c|c|c|c|c|c|c|c|c|c|}
\hline Number. & 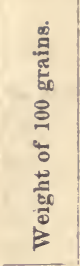 & 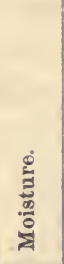 & कू & 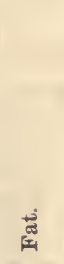 & 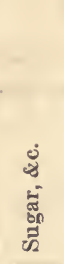 & 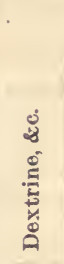 & 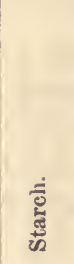 & 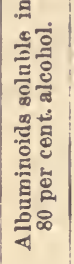 & 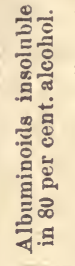 & 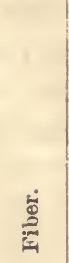 & 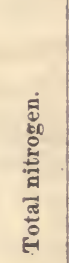 & 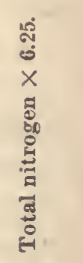 \\
\hline $11 \ldots$ & $\begin{array}{l}2.090 \\
1.756 \\
1.798 \\
1.878 \\
1.684 \\
1.892 \\
1.495 \\
1.922 \\
1.780 \\
2.139 \\
1.703 \\
1.648 \\
1.6016 \\
1.656 \\
1.506 \\
1.595 \\
1.313 \\
1.355\end{array}$ & $\begin{array}{l}6.32 \\
5.93 \\
6.57 \\
8.00 \\
7.38 \\
6.08 \\
7.07 \\
7.21 \\
6.95 \\
7.28 \\
6.34 \\
6.99 \\
6.78 \\
6.77 \\
7.00 \\
6.13 \\
8.75 \\
6.95\end{array}$ & $\begin{array}{l}2.25 \\
2.38 \\
2.02 \\
2.17 \\
2.61 \\
3.07 \\
2.38 \\
1.95 \\
2.45 \\
1.78 \\
2.03 \\
2.43 \\
2.07 \\
2.20 \\
2.06 \\
2.35 \\
2.15 \\
1.60\end{array}$ & $\begin{array}{l}7.86 \\
8.25 \\
8.64 \\
4.48 \\
7.60 \\
7.83 \\
7.23 \\
815 \\
8.21 \\
8.52 \\
6.98 \\
7.75 \\
7.40 \\
8.88 \\
8.12 \\
8.58 \\
9.47 \\
7.77\end{array}$ & $\begin{array}{l}5.59 \\
6.21 \\
5.84 \\
6.02 \\
5.96 \\
5.67 \\
5.67 \\
5.66 \\
6.56 \\
5.80 \\
6.28 \\
6.39 \\
6.10 \\
6.52 \\
5.43 \\
6.43 \\
6.50 \\
6.69\end{array}$ & $\begin{array}{l}3.68 \\
3.68 \\
3.12 \\
3.96 \\
.40 \\
2.82 \\
3.90 \\
3.56 \\
4.52 \\
3.86 \\
3.8 .2 \\
3.78 \\
3.42 \\
3.60 \\
3.42 \\
3.48 \\
3.86 \\
3.58\end{array}$ & $\begin{array}{l}58.87 \\
59.04 \\
58.34 \\
58.29 \\
60.81 \\
60.18 \\
58.47 \\
58.24 \\
53.56 \\
58.08 \\
54.92 \\
56.17 \\
53.69 \\
56.25 \\
54.31 \\
56.64 \\
56.08 \\
52.59\end{array}$ & \begin{tabular}{l|}
.97 \\
1.69 \\
1.50 \\
1.72 \\
1.86 \\
2.15 \\
1.95 \\
1.36 \\
2.43 \\
2.20 \\
1.42 \\
2.71 \\
2.78 \\
$(?)$ \\
1.15 \\
2.31 \\
2.70 \\
1.83
\end{tabular} & $\begin{array}{r}13.56 \\
11.26 \\
12.68 \\
14.21 \\
12.32 \\
11.15 \\
11.88 \\
12.64 \\
14.02 \\
11.28 \\
16.62 \\
12.55 \\
16.66 \\
14.53 \\
17.05 \\
12.57 \\
9.20 \\
17.42\end{array}$ & $\begin{array}{l}.90 \\
1.56 \\
1.29 \\
1.15 \\
1.08 \\
1.05 \\
1.45 \\
1.23 \\
1.30 \\
1.20 \\
1.60 \\
1.23 \\
1.10 \\
1.25 \\
1.46 \\
1.51 \\
1.29 \\
1.57\end{array}$ & $\begin{array}{l}2.32 \\
2.07 \\
2.27 \\
2.55 \\
2.27 \\
2.13 \\
2.21 \\
2.24 \\
2.63 \\
2.16 \\
2.88 \\
2.44 \\
3.11 \\
2.32 \\
2.91 \\
2.38 \\
1.90 \\
3.08\end{array}$ & $\begin{array}{l}14.53 \\
12.95 \\
14.18 \\
15.93 \\
14.18 \\
13.30 \\
13.83 \\
14.00 \\
16.45 \\
13.48 \\
18.04 \\
15.26 \\
19.44 \\
14.53 \\
18.20 \\
14.88 \\
11.90 \\
19.25\end{array}$ \\
\hline
\end{tabular}

BARLEY.

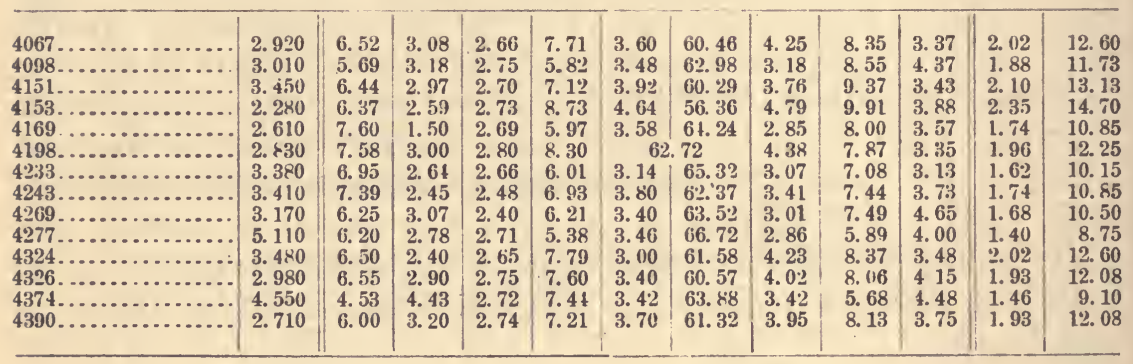

RYE.

\begin{tabular}{|c|c|c|c|c|c|c|c|c|c|c|c|c|}
\hline & 516 & & 88 & 80 & 10 & 76 & 52 & 2. 20 & 7.60 & 1.45 & 1.57 & \\
\hline & & & 01 & 2.17 & 7.93 & & 60.47 & 3. & & & & 12.95 \\
\hline & 840 & 8.45 & 2. 36 & 1.98 & 8.4 & & & 3. & & & & 13.13 \\
\hline e. & 1.670 & 9.18 & 1.62 & 1. 70 & 6. 25 & 4.5 & 64. & 2. & 9.1 & & & \\
\hline 5107 & 2.034 & 8.32 & 2. 08 & 1.93 & 692 & 4. 54 & 63. & 2. & 9. 24 & 1.28 & & \\
\hline 5116 & 2.250 & 8.85 & 2. 06 & 1.93 & 7. 81 & 5. 54 & 60.35 & 3. & 8. 97 & 1.38 & 1.93 & \\
\hline 5140 & 2.164 & 9. 70 & 2.10 & 1.93 & 7. 29 & 5. 32 & 60.55 & 2.44 & & & & \\
\hline 15 & 2.670 & 8.93 & 2. 03 & 1.74 & 6. 20 & 6. 02 & 62.12 & 1.76 & & & & \\
\hline 231 & 2.310 & 8. 98 & 2. 16 & 1.69 & 7.85 & 5.19 & 61.33 & 2. 17 & & & & \\
\hline 48 & 1.873 & 8.75 & 2.01 & 1.85 & 7.52 & 4. 20 & 6. 74 & 2. 18 & & 1.55 & & \\
\hline 269 & 2.080 & 8.15 & 1.70 & 1.93 & 7. 89 & 4. 14 & & 2.71 & 9. 37 & & 1.93 & \\
\hline $5 \geq 8$ & 2.422 & 9.35 & 2.15 & 1.86 & 9.46 & 4.44 & 59.81 & $3.0 R$ & & 1.20 & & \\
\hline $5 \div 9$ & 2.154 & 9.75 & 2.10 & 1.70 & 6. 74 & 4. 36 & 63.31 & 1.90 & 5 & 1. 89 & & \\
\hline & 2.064 & 8.35 & 2. 68 & 1.75 & 7. 89 & 4.44 & 58.73 & 3. 0.3 & 9. 05 & 1.54 & & \\
\hline 535 & 2.012 & 8.65 & 2. 32 & 1.86 & 7. 10 & 5. 00 & 62.40 & 276 & 8.44 & 1. & 1. 79 & 11. \\
\hline & 1.087 & 8. 80 & 1.96 & 1.84 & 7.45 & 4.46 & 62. 59 & 2. 56 & 8.99 & 1. & 1. 85 & 11.55 \\
\hline 5360 & 1.846 & 8.38 & 1.90 & 1.38 & 7.83 & 4. 80 & 62. 19 & 2. 15 & 9. 39 & 1.56 & 1.90 & 11.90 \\
\hline
\end{tabular}


The sources of the specimens will be found under their respective serial numbers in the general descriptice tables. They are from various parts of the country, and represent fairls the average production and variations.

For comparison, averages of the above results have been drawn, as well as of those of wheat and corn previously published, excluding the Colorado wheats.

Avernges of detailed analyses of cereals.

\begin{tabular}{|c|c|c|c|c|c|}
\hline & Wheat. & Corn. & Oats. & Barley. & Ryө. \\
\hline No. of aualyses .. & 27 & 21 & 18 & 14 & 17 \\
\hline 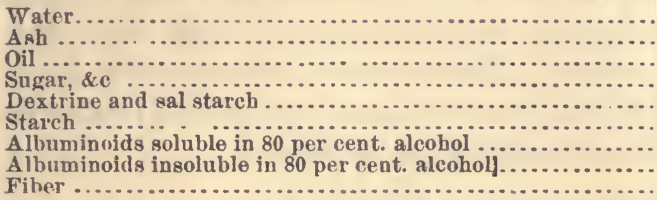 & \begin{tabular}{|r|}
9.25 \\
1.84 \\
2.30 \\
3.50 \\
2.30 \\
67.88 \\
3.58 \\
7.45 \\
1.90
\end{tabular} & $\begin{array}{r}9.34 \\
1.54 \\
5.54 \\
2.18 \\
2.18 \\
66.91 \\
5.84 \\
4.96 \\
1.41\end{array}$ & $\begin{array}{r}6.92 \\
2.22 \\
7.87 \\
6.07 \\
3.47 \\
56.91 \\
1.82 \\
13.43 \\
1.29\end{array}$ & \begin{tabular}{r|}
6.47 \\
2.87 \\
2.67 \\
7.02 \\
3.55 \\
62.09 \\
3.66 \\
7.86 \\
3.81
\end{tabular} & $\begin{array}{r}8.85 \\
2.08 \\
1.83 \\
7.57 \\
4.75 \\
61.87 \\
2.53 \\
9.07 \\
1.47\end{array}$ \\
\hline & 100.00 & 100.00 & 100.00 & 100.00 & 100.00 \\
\hline Total albuminoids. & 11.03 & 10.80 & 14.25 & 11.52 & 11.60 \\
\hline
\end{tabular}

From the figures it is seen that oats and barley are much drier than the remaining cereals. This is due in the case of both to the outer chaffy covering, which readily gives up its water. In the oats, however, this had been removed, but its effect in abstracting moisture has evidently remained. The smaller size of the rye kernel, no doubt, accounts for its somewhat lower moisture than wheat and corn, and this, too, has perhaps an effect upon oats.

Of all the grains barley is the richest in ash-this, too, probably due to its hull-followed by oats, the richest actually in ash in kernel.

In oil, oats is alead of corn by over 2 per cent., and far ahead of all the other cereals.

In sugar, barley and rye are superior, with oats comparatively rich, and corn the poorest.

In dextrine or gum, rye is the richest, having twice as much as wheat and corn, and 1 per cent. more than oats and barley.

The accumulation of its many other constituents makes oats by far the least starchy of the cereals, followed by rye and barley, with wheat as the most starchy: This latter fact, from a flouring point of view, is important, taken in connection with the character of the nitrogen of wheat and its small amount of oil. Of the determinations of the nitrogenous constituents it must be said that the solubility does not show much in regard to their quality. Part of the gluten of wheat goes into the alcohol extract and part remains insoluble, the latter being chiefly the gluten-casien. In corn the soluble portion is known often as zein and is more distinctive than the soluble albuminoids of the other cereals. 
It is the largest in amount of the soluble nitrogenous constituents found in any of the grains, exceeding the insoluble portion. Oats, on the other hand, contains the least soluble albumen.

Barley is, in the condition in which it was analyzed, most fibrous, but in its hulless state no more so than wheat. Aside from this the most fiber is found in wheat and the least in oats. From the averages wide variations will be found anong individual analyses, which are due to circumstances of environment which our data do not at present permit a study of. A large increase of analyses and conditions which may in time accumulate will render this possible.

\section{MILLING PRODUCTS.}

In our secoud report an examination of the products of roller milling was presented, with especial reference to the process as applied to the hard spring wheats of the North west. This examination has since been extended to the winter wheats of Kausas, as represented by the products of a small roller mill at Ottawa. The data are as follows :

Mill products from Ottawa, Kans.

\begin{tabular}{|c|c|c|c|c|c|c|c|c|c|}
\hline Description of sample. & 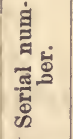 & 兽 & वี & $\ddot{0}$ & 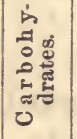 & 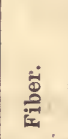 & $\frac{\text { aี }}{\text { है }}$ & 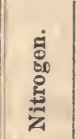 & 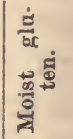 \\
\hline Vhol & $\begin{array}{r}\text { Pr.ct. } \\
6014\end{array}$ & $\begin{array}{r}P r . c t . \\
9.55\end{array}$ & $\begin{aligned} & P r \text { ct. } \\
& 1.78\end{aligned}$ & $\begin{array}{r}P r . c t . \\
1.27\end{array}$ & $\begin{aligned} & P r . \text { ct. } \\
& 72.97\end{aligned}$ & $\begin{array}{r}\text { Pr.ct. } \\
1.88\end{array}$ & Pr.ct. & $\begin{array}{r}P r . c t . \\
1.96\end{array}$ & Pr. ct. \\
\hline irst & 6015 & 8. 68 & 1. 90 & 2.22 & 72.67 & 1.93 & & 2.02 & 31.74 \\
\hline cond break. & 6016 & 9.15 & 1. & 2.17 & 71.57 & 2.48 & 12.78 & 2.04 & 27. \\
\hline J & 6017 & 9.30 & 2. & 2.16 & 71.19 & & & 2.07 & 27. \\
\hline Fon & 6018 & 8.18 & 2.55 & 2. 62 & 70.63 & 2. 77 & 13 & 2.18 & 24.90 \\
\hline Fift & 6019 & 9.40 & 4. & 3.41 & 63. & 4.00 & 15 & 2.44 & t \\
\hline - & 6020 & 7.60 & 5.20 & 3. 99 & 62 & 5. 60 & & 2.41 & \\
\hline Bra & 6021 & 8.45 & 6. & 4. 64 & 58. & 6.60 & 15 & 2. 5\% & \\
\hline or & 6022 & 8.18 & 3.38 & 5. 65 & 62.45 & 2.84 & & 2.80 & \\
\hline Cho & 6023 & 11.40 & .78 & 2.00 & 73. & 1.23 & 11 & 1.76 & 26.14 \\
\hline Fi & 6024 & 11.43 & .38 & 1.11 & 75. & .42 & & 1.82 & 29.10 \\
\hline Medinm nid & 6025 & 11.03 & .50 & 1.29 & 74.93 & 1.40 & 10.85 & 1.74 & 28. 76 \\
\hline Coarso milldlings...... & 6026 & 8.88 & .83 & 1. 84 & 77.25 & .70 & 10.50 & 1.68 & \\
\hline Ger & 6027 & 10.75 & 2. 30 & 3.99 & 67.09 & 1. 69 & 14 & 2.27 & \\
\hline Tail & 6028 & & & & & & & & \\
\hline Fin & 6029 & 8. 70 & 5. & 7. & 54. & 3. 59 & & 3. 22 & \\
\hline from thir & 6030 & 11. 35 & & & & & & 1.79 & 28.45 \\
\hline Fin & 6031 & 11. 25 &. & 6. 05 & 71. & .46 & & 1.74 & 25. \\
\hline Sec & 6032 & 11.30 & & 1.15 & & .40 & & & 27.75 \\
\hline Flo & 6033 & 10.85 & 3 & .93 & 78. & .12 & 9. & 1.48 & 23.79 \\
\hline Flonr fromi fir & 6034 & 11. 50 & & .96 & 78. 13 & .85 & 7.88 & 1. 26 & 19.28 \\
\hline d, third, fourth, and & 6035 & 11.42 & .43 & 1.06 & 76. 89 & .22 & 9.98 & 1.60 & 27: 5 \\
\hline Stra & 6036 & 11. & 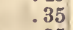 & 1.01 & 76. & .25 & 10. & 1. 52 & 25.65 \\
\hline Pat & 6037 & 11. & & 1.01 & 77. & .19 & & 1.54 & 22.07 \\
\hline & 6038 & 10.60 & .80 & 2. 09 & 72 . & .52 & 13. & & 41. \\
\hline Flour from third middlings... & 6039 & 12.48 & .32 & 1.17 & 73.75 & .20 & 12.08 & 1.93 & 33.83 \\
\hline
\end{tabular}

The winter wheat from which the Kansas products were obtained is a larger and softer grain than the spring varieties of Minnesota. It is much less oily than the usual production of the country at large, and while above the average in nitrogenous constituents and representing about the usual percentage found in that portion of the country where it was grown, it is of course lower in albuminoids than the Minnesota grain. The relative results in the breaks are, however, about the same 
in both cases. There is a somewhat greater accumulation of ash and fiber in the last breaks, and the shorts are proportionately richer in nitrogen. This is hardly what would be expected in milling softer wheats, but shows that the process is as effectire as with spring varieties in separating the endosperm or floury portion of the grain from the outer coats. The increase in the ash and fiber may be due, however, to a larger proportion of germ in the branny products than is the case in Minnesota.

The grading and puritication of the middlings in the two mills is so different that it is difficult to make a comparison of their relative purity. The finest middlings are in both cases the most impure, carrying the most bran and germ, and consequently being rich in nitrogen. The tailings from the purification of these middlings are largely germ and bran, and are very rich in ash, oil, and albuminoids. The flour from the fine middlings is richer in both cases in nitrogen, owing to its contamination with germ and bran, and the best is produced from the coarse. There is a somewhat less relative proportion of the total albuminoids of the wheat in the coarse middlings flour of winter than of spring wheat, a condition which is also observed in the finished produets, patent and straight grades. The low grade of the Kansas mill will be seen to be quite different from that so called in Minnesota. It is, it seems, more nearly what is there known as a baker's grade. The low grade from spring wheat, while rich in nitrogen from the amount. of bran and germ it contains, is very poor in gluten. The baker's grade from that grain is not only rich in nitrogen but also in gluten; with this the low-grade Kansas flour corresponds, due probably to the fact that in the smaller mill the process of refinement is not earried to such extremes. In the finished germ there is also visible a vast difference. It is not separated in as clean and entire condition from the winter wheat, either owing to lack of facilities or difficulties in the way, and is consequently less rich in nitrogen. The break flours present, too, as. great a contrast as the low grade, that from Kansas being much more. starchy and less glutinous or stiff. It is of better quality, for, although poor in gluten, it does not containing as much germ.

Depending largely on the original wheat, the finished products in Kansas are not as stiff as those from spring grain, but this was to be expected, and the greatest differences which have been observed are due fully as much, as far as we can judge, to method of manufacture as to physical differences in the grain. That there are some to be ascribed to this cause is evident from the lower relation of the purer products in Kansas to the original grain. This was observed also in. roller flours from Ohio and Washington, D. C., in our former inrestigatious.

There seems to be every advantage over ordinary milling with stones. in the use of this process with our winter wheats, both. in economy and. quality, although, perhaps, not as great as with the hard. Northwestern. 
grain, uor as large in small as in, large mills. The rapid extension of the system shows that practice has demonstrated what chemical and physical investigations have shown to be advisable, and it seems from a comparison of our analyses of the two series that the more the products are differentiated the more satisfactory are the results.

In another place an investigation of the proximate composition of the germ will soon be published, showing the presence of remarkably large amounts of cane sugar, together with another sugar not yet identified, and proving not to be raffinose; and of allantoin, a nitrogenous substance hitherto found only once as a plant constituent and whose presence is of great importance from a botanico-physiological point of view.

\section{MISCELLANEOUS ANALYSES OF CEREALS.}

In the routine work of the division many miscellaneous samples of cereals have been from time to time examined. The results are here collected. The sources from which they have been derived are as follows :

\section{Sources of specimens.}

WHEAT.

2763. From New Zealand. Selected sample. Crop of 1884.

2764. From Mr. MeLanghlin, New Jerusalem, Ventura County, California. Crop of 1884 .

2765. From Charles Woodhull, Wahpeton, Dak. Crop of 1884.

CORN.

1978. Bessarabian. From S. M. Clark, Washington, D. C. Crop of 1884.

2438. From T. J. Higgins, Morris County, Kansas. A fine large-eared Yellow Dent. 2428. From S. M. Clark, Washington, D. C. Crop raised from seed 2438. This sample tho less perfect and most shriveled kernels of one cob. Crop of 1884 .

2429. Froin S. M. Clark, Washington, D. C. The perfect and plump kernels of the above-mentioned cob.

2430. From Lake County, Tennessee. 1883. White Dent.

2431. From Giles County, Temmessee. 1883. White Dent.

24:3. From Bradley County, Tennessee. 1883. White Dent.

2433. From Clay County, Kentucky. 1883. White Dent.

2434. From Brown County, Minuesota. 1883. White Dent.

2435. From Herkimer County, New York. 1883. Yellow Flint.

2436. From Jackson County, Minnesota. 1883. Red Dent.

2437. From Watonwan County, Minnesota. 1883. Yellow Dent.

2440. From —, Florida. 1884. Yellow Dent.

OATS.

1973-5. From the collection of the Atchison, Topeka aud Santa Fé Railroad. Grown in Kansas.

1976. From J. P. Hooke, Marysville, Tenn. Winter variety.

1977. From R. B. Potter, M. D., Dade County, Florida.

2766. Distributed by the Department. 1885. Welcome Oats.

2766. Distributed by the Department. 1835. Clydedale Oats.

3000. From New Zealand. 40 pounds per bushel. Crop of 1884 . 
RYE."

1971-2. From the collection of the Atchison, Topeka and Santa Fé Railroad. Grown in Kansas.

BA RLEY.

1978. Distributed by the Department. 1884. Imperial.

The :malyses are as follows:

Mixcellaneous analyse's, 1884-'8:.

\begin{tabular}{|c|c|c|c|c|c|c|c|c|c|c|}
\hline Cereals. & 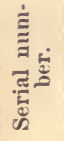 & Locality. & 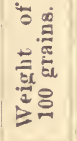 & $\stackrel{\frac{b}{\pi}}{\frac{5}{\sigma}}$ & $\frac{\pi}{4}$ & تีं & $\begin{array}{l}\therefore . \\
\approx 0 \\
\approx \\
\vdots \\
0 \\
0 \\
0\end{array}$ & 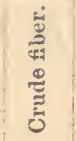 & 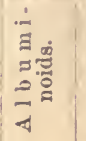 & 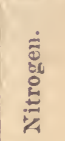 \\
\hline \multirow[t]{3}{*}{ Wheat.................. } & 2763 & New Zealaud & 5. 036 & 9. 40 & 1.25 & 1. 76 & 71.49 & 1.75 & 14.35 & 2.30 \\
\hline & 2764 & California & ..... & 7.00 & 2.45 & 4.95 & 71.32 & 2.90 & 11.38 & 1.82 \\
\hline & 2765 & Dakota & ....... & 8.55 & 1. & 3. 01 & 71.51 & 1.95 & 13.13 & 2.10 \\
\hline \multirow{13}{*}{ Corn (maize) kernel... } & 1978 & Dist. of Columbia. & ....... & 8.70 & 1.75 & 4.44 & 72.67 & 1.24 & 11.20 & 1.79 \\
\hline & 2428 & Dist. of $\mathrm{Cu}_{\mathrm{u}}$ & & 10.75 & & & 75.52 & 1.80 & 7.00 & \\
\hline & 2429 & Dist. of Columbia. & & 10.12 & 1.35 & 4. 32 & 75.51 & 1.70 & 7.00 & 1.12 \\
\hline & $2+38$ & Kansas .... & & 10.00 & 1.50 & 4. 37 & 71.38 & 1.90 & 10.85 & \\
\hline & 2430 & Tenr & & 11.15 & 2. 05 & 4. 34 & 72.18 & 1.70 & 8.58 & 1. 37 \\
\hline & 2431 & & & 9.65 & 2. & 4.95 & 71.32 & 2.40 & & \\
\hline & $24: 32$ & Teni & & 10.45 & 1.65 & 4. 57 & 71.73 & 2.15 & 9.45 & 1.51 \\
\hline & $\mathbf{2 4 3 3}$ & Kentucky & & 10.55 & 1. & 4. 17 & 73.10 & 1.90 & & \\
\hline & 2434 & Minnesota & & 9.60 & 1. 75 & 4. 53 & 72.55 & 2.12 & 9. 45 & 1. 51 \\
\hline & 2435 & New York & & 850 & 1.45 & 4. 51 & 71.62 & 2.02 & 11.90 & \\
\hline & 2436 & Minnesota & & 9.80 & $\therefore .00$ & 4.01 & 71.76 & 2.10 & 10.33 & 1. 65 \\
\hline & 2437 & Minne'sota.. & & 10.50 & 1. 65 & 4. 05 & 71.40 & 2. 07 . & 10.33 & \\
\hline & 2440 & Florida .... & & 8.47 & 1. & 4.82 & 72.99 & 2. 02 & 10.33 & 1. 65 \\
\hline \multirow{9}{*}{ Oats $\ldots . . . \ldots \ldots \ldots$} & 1973 & Kan & & 8. 76 & 2.55 & 7.15 & 65. & .96 & 15.58 & 2.49 \\
\hline & 1974 & Kansas .......... & & 8.87 & 2.60 & 5. 79 & & 39 & 14. 35 & 2. 32 \\
\hline & 1975 & Kansas .... . & & 8. 37 & 2.75 & 8.14 & & & 16.63 & 2. 66 \\
\hline & 1976 & Tenuessce. & & 9. 0.3 & 2. 09 & 9.42 & 63. & 71 & 15.75 & 2.52 \\
\hline & 1977 & Florida & & 9.07 & 2.45 & 9.43 & & .22 & 13.83 & \\
\hline & 2766 & Imported .. & & 9.60 & 2.40 & 8. 83 & & 82 & 14.35 & 2.30 \\
\hline & & & & & & 9.02 & & & & \\
\hline & 3000 & New Zea & & *10.18 & *2. 32 & $\bullet 8.91$ & $\because 65.34$ & $\star 1$ & $\star 11.55$ & ${ }^{\star} 1.85$ \\
\hline & & Zeala & & 17.60 & & t. 80 & & $\dagger 20$. & $\div 2$. & \\
\hline $\mathbf{r}$ & 1971 & Kansas .... & & 11. 60 & 1.60 & 2.22 & 70.49 & 1.49 & 12.60 & 2. \\
\hline & 1972 & Kansas.... & & 11.20 & 1. 80 & $1.8\}$ & 71. 32 & 1.40 & 12.40 & 1. \\
\hline Barley. & 1984 & ? $\ldots \ldots$ & & 8.46 & 2.45 & 2. 71 & 73.01 & 1. 82 & 11.55 & 1.85 \\
\hline
\end{tabular}

It is unnecessary to call particular attention to these analyses. The New Zealand wheat was a selected exhibition sample, rery large and heavy, and of rather a soft character. It is rather poor in ash and oil, and quite rich in albuminoids.

The specimens from California and Dakota sustain the usual reputation of the grain from those States.

The samples of corn vary very little in composition for the different localities. The only exception which it is of interest to observe is in the case of the plump and shriveled grains grown in the District of Uolunıia from seed produced in Kansas. While not varying at all among themselves in composition, the plump and shriveled grains have departed quite remarkably from the composition of the parent seed from Kansas. Among the remaining specimens the usual narrow limits of variation in this cereal are seen. Even Minnesota, which produces a wheat rich in nitrogen, does not excel in its corn in this respect.

The analyses of oats show no peculiarities; the sample from New 
Kealand alone is rather low in albuminoids and not corresponding to the wheat with which it was associated. The kernels were analyzed free from hulls.

\section{ANALYSIS OF WHEAT'S OF THE CROP OF 1855.}

Recently several Minnesota wheats, harvested during the season of 1885, have been examined. They sustain the averages of previous years, or are slightly richer in albuminoids. The results follows together with those of seed wheats distributed by the Department in the autumn of 1585 , the products of which it is hoped may be compared another year.

Analysis of wheats from Minnesota, 1885.

\begin{tabular}{|c|c|c|c|c|c|c|c|c|c|c|}
\hline Variety. & 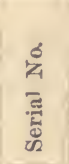 & 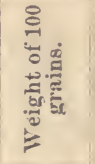 & 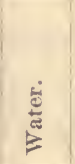 & बี & $\bar{\sigma}$ & & 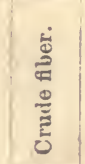 & 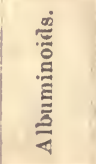 & 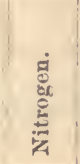 & 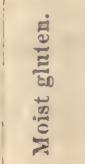 \\
\hline $\begin{array}{l}\text { Rice. } \\
\text { Blue Stem } \\
\text { Scotch Fife. } \\
\text { Sicotch Fife. } \\
\text { Sicoteb Fife }\end{array}$ & $\begin{array}{l}6000 \\
6001 \\
6002 \\
6003 \\
6004\end{array}$ & \begin{tabular}{r|} 
Grains. \\
4.114 \\
3.096 \\
2.619 \\
2.190 \\
2.565
\end{tabular} & \begin{tabular}{r|} 
Perc. \\
9.40 \\
9.13 \\
918 \\
9.38 \\
8.93
\end{tabular} & $\begin{array}{l}\text { Per } c . \\
2.08 \\
2.03 \\
2.43 \\
1.70 \\
2.03\end{array}$ & $\begin{array}{l}\text { Perc. } \\
2.79 \\
2.94 \\
2.65 \\
2.98 \\
2.89\end{array}$ & \begin{tabular}{l|} 
Per $c$. \\
70.33 \\
69.48 \\
70.47 \\
69.06 \\
68.75
\end{tabular} & \begin{tabular}{l|} 
Per $c$. \\
1.92 \\
2.49 \\
1.79 \\
1.83 \\
2.00
\end{tabular} & $\begin{array}{c}\text { Per } c . \\
13.48 \\
14.00 \\
13.48 \\
15.05 \\
15.40\end{array}$ & $\begin{array}{l}\text { Perc. } \\
2.16 \\
2.14 \\
2.16 \\
2.41 \\
2.46\end{array}$ & $\begin{array}{r}\text { Perc. } \\
29.37 \\
29.41 \\
32.31 \\
32.67 \\
38.45\end{array}$ \\
\hline
\end{tabular}

Analysis of uinter wheats distributed by the Department, November, 1885.

\begin{tabular}{|c|c|c|c|c|c|c|c|c|c|c|c|}
\hline Variety. & 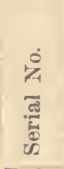 & 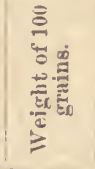 & 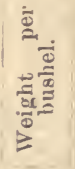 & 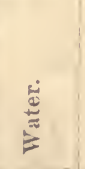 & क्ष & 5 & 㺼 & 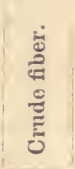 & 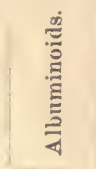 & 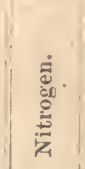 & 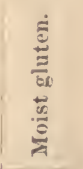 \\
\hline 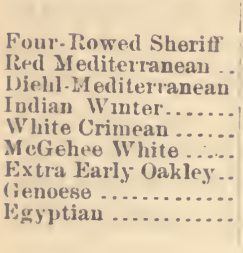 & $\begin{array}{l}6005 \\
6006 \\
6007 \\
6008 \\
6009 \\
6010 \\
6011 \\
6012 \\
6013 \\
6040\end{array}$ & $\begin{array}{c}\text { Grains. } \\
3.734 \\
4.635 \\
4.222 \\
3.126 \\
6.086 \\
2.990 \\
3.945 \\
4.113 \\
5.308 \\
(?)\end{array}$ & \begin{tabular}{|c|}
$L b 8$. \\
65.8 \\
67.2 \\
65.8 \\
65.5 \\
66.5 \\
66.4 \\
66.3 \\
68.7 \\
68.6 \\
$(?)$
\end{tabular} & $\begin{array}{r}\text { Per } c . \\
10.28 \\
11.05 \\
10.45 \\
10.2 J \\
10.73 \\
10.48 \\
11.45 \\
9.68 \\
9.83 \\
9.88\end{array}$ & $\begin{array}{l}\text { Per c. } \\
1.95 \\
1.63 \\
1.65 \\
9.23 \\
2.00 \\
1.88 \\
1.20 \\
1.73 \\
1.78 \\
1.90\end{array}$ & $\begin{array}{l}\text { Per } c \text {. } \\
2.29 \\
2.22 \\
2.25 \\
2.06 \\
2.55 \\
2.22 \\
2.29 \\
2.04 \\
2.04 \\
2.40\end{array}$ & $\begin{array}{r}\text { Per } c . \\
73.52 \\
71.25 \\
72.97 \\
72.24 \\
70.63 \\
71.34 \\
69.79 \\
74.68 \\
74.52 \\
72.11\end{array}$ & $\begin{array}{l}\text { Per } c . \\
1.81 \\
1.60 \\
2.00 \\
1.72 \\
1.66 \\
1.83 \\
1.79 \\
1.89 \\
1.50 \\
1.46\end{array}$ & $\begin{array}{r}P e r \\
10.15 \\
12.25 \\
10.68 \\
11.55 \\
12.43 \\
12.25 \\
13.48 \\
9.98 \\
10.33 \\
12.25\end{array}$ & $\begin{array}{l}\text { Per } c- \\
1.62 \\
1.96 \\
1.72 \\
1.85 \\
1.99 \\
1.96 \\
2.16 \\
1.60 \\
1.65 \\
1.96\end{array}$ & $\begin{array}{l}\text { Per } c \text {. } \\
26.54 \\
31.66 \\
32.22 \\
19.16 \\
00.00 \\
33.61 \\
32.05 \\
22.61 \\
29.43 \\
32.04\end{array}$ \\
\hline
\end{tabular}

\section{CONCLUSION.}

The results which have been collected and discussed in this and pre vious reports have shown the wide extent of the variations which occur in the phrsical and chemical properties of our cereal grains. They have extended over but a few years, and with conditions which have not been sufficiently varied or sufficiently under control. They have served to show, however, how many of the modify ing causes are in the hands of the furmer or of the experimeutal stations, and, to a certain extent, the directions in which advance should be made. The co-operation of practical field.work is now necessary, with laboratory examinations of the results. Until this acemplished systematically further progress will be slow grimbigertinily. 


